\title{
ON THE DEFORMATION OF ALGEBRA MORPHISMS AND DIAGRAMS
}

\author{
BY \\ M. GERSTENHABER ${ }^{1}$ AND S. D. SCHACK
}

\begin{abstract}
A diagram here is a functor from a poset to the category of associative algebras. Important examples arise from manifolds and sheaves. A diagram $\mathbf{A}$ has functorially associated to it a module theory, a (relative) Yoneda cohomology theory, a Hochschild cohomology theory, a deformation theory, and two associative algebras $\mathbf{A}$ ! and $(\# \mathbf{A})$ !. We prove the Yoneda and Hochschild cohomologies of $\mathbf{A}$ to be isomorphic. There are functors from $\mathbf{A}$-bimodules to both $\mathbf{A}$ !-bimodules and $(\mathbf{A})$ !bimodules which, in the most important cases (e.g., when the poset is finite), induce isomorphisms of Yoneda cohomologies. When the poset is finite every deformation of (\#A)! is induced by one of $\mathbf{A}$; if $\mathbf{A}$ also takes values in commutative algebras then the deformation theories of $(\mathbf{A})$ ! and $\mathbf{A}$ are isomorphic. We conclude the paper with an example of a noncommutative projective variety. This is obtained by deforming a diagram representing projective 2 -space to a diagram of noncommutative algebras.
\end{abstract}

0. Introduction. There is a striking similarity between the formal aspects of the deformation theories of complex manifolds and associative algebras. In this work we link the two with a deformation theory for diagrams and prove a Cohomology Comparison Theorem (CCT) which partially explains the analogy. The CCT enables one to show-among other things - that the deformation theory of a diagram associated to a compact manifold is isomorphic to that of a certain associative algebra. The assignment diagram $\leadsto$ algebra is functorial while manifold $\leadsto$ diagram is not. (The CCT has much wider applications; for example, we sketch here (\$7), and will discuss in detail in a later paper, its application to simplicial cohomology.) Here are the basic definitions:

We fix a commutative unital ring $k$ and consider the category ALG of associative unital $k$-algebras. All algebras and bimodules over them are required to be symmetric $k$-modules, i.e. the left and right actions coincide. Tensor products will always be taken over $k$ unless otherwise indicated.

Received by the editors October 13, 1981 and, in revised form, October 12, 1982.

1980 Mathematics Subject Classification. Primary 16A58, 16A61; Secondary 18G10, 18G25, 55N35, $14 \mathrm{~F} 05$.

Key words and phrases. Associative algebra, diagram, Hochschild cohomology, Yoneda cohomology, deformation, simplicial cohomology.

${ }^{1}$ The first author wishes to thank the Institute for Advanced Study for a membership during the academic year 1980-1981 and gratefully acknowledges the support of the N.S.F. through a grant to the University of Pennsylvania. A preliminary version (with some errors) was presented at the Conference in Algebra in honor of Nathan Jacobson, held at Yale University, June 2-5, 1981, sponsored by the N.S.F. Proceedings of the conference appear in Algebraist' Homage, Contemporary Mathematics Vol. 13, Amer. Math. Soc., Providence, R.I., 1982. 
Let $I=\{i, j, k, \ldots\}$ be a partially ordered set ( poset). (The double use of $k$ as ground ring and index should cause no confusion.) View $I$ as the object set of a category in which there is a unique morphism $i \rightarrow j$ whenever $i \leqslant j$. A (commutative) diagram of algebras over $I$ is a (contravariant) functor A: $I^{\text {op }} \rightarrow$ ALG. (A is called finite if $I$ is finite.) We simplify notation by writing $\mathbf{A}^{i}$ and $\varphi^{i j}: \mathbf{A}^{j} \rightarrow \mathbf{A}^{i}$ for $\mathbf{A}(i)$ and $\mathbf{A}(i \rightarrow j)$. Then $\varphi^{i j} \varphi^{j k}=\varphi^{i k}$ whenever $i \leqslant j \leqslant k$ and $\varphi^{i i}$ is the identity morphism of $\mathbf{A}^{i}$. Objects such as complex manifolds, varieties, arbitrary schemes (as well as morphisms between them) are equivalent to certain diagrams of commutative algebras. For example $I$ may be the set of coordinate neighborhoods of a complex manifold $\mathcal{X}$, partially ordered by inclusion, with $\mathbf{A}^{i}$ the ring of holomorphic functions on $i$ and $\varphi^{i j}$ the restriction morphism; this information determines $x$. When $\mathcal{X}$ is compact we may choose a finite open covering by Stein neighborhoods with the property that the intersection of any two is again in the cover. The associated finite diagram still completely describes $x$.

$\mathbf{A}$ (formal) deformation of $\mathbf{A}$ will be a factorization $\mathbf{A}_{t}$ of $\mathbf{A}$ through the category of deformed $k$-algebras. That is, each $\mathbf{A}_{t}^{i}$ is a deformation of $\mathbf{A}^{i}$ in the sense of [G2] (so as modules over $k_{t}=k[[t]]$ we have $\left.\mathbf{A}_{t}^{i}=\mathbf{A}^{i}[[t]]\right)$; also each $\varphi_{t}^{i j}$ reduces modulo $t$ to $\varphi^{i j}$, and so may be expressed as a power series $\varphi_{t}^{i j}=\varphi^{i j}+t \varphi_{1}^{i j}+t^{2} \varphi_{2}^{i j}+\cdots$, where each $\varphi_{m}^{i j}: \mathbf{A}_{t}^{j} \rightarrow \mathbf{A}_{t}^{i}$ is the $k_{t}$-linear extension of a $k$-module morphism $\mathbf{A}^{j} \rightarrow \mathbf{A}^{i}$. When the diagram $\mathbf{A}$ comes from a complex manifold $\mathfrak{X}$ then a formal deformation of $\mathfrak{X}$ induces a deformation of $\mathbf{A}$. The converse is true for deformations of $\mathbf{A}$ in which the $\mathbf{A}^{i}$ remain commutative. Similar relations hold in some other cases and probably-in some sense not yet explicit-in general.

Our main results are these: There is a functor assigning to each diagram $\mathbf{A}$ a category of A-bimodules, A-MOD. (A is itself an A-bimodule.) When every $\mathbf{A}^{i}$ is commutative there is a distinguished subcategory of symmetric A-bimodules. (Again, $\mathbf{A}$ is an example.) Whenever we are discussing modules from this subcategory we shall say that we are "in the commutative case." Now A-MOD is abelian and carries a natural "relative" Yoneda cohomology bifunctor $\operatorname{Ext}_{\mathbf{A}}^{*}(-,-)$. On A-MOD we also define "Hochschild" cochain complexes $C^{*}(\mathbf{A},-)$ whose cohomology we denote $H^{*}(\mathbf{A},-)$. We establish a natural isomorphism $H^{*}(\mathbf{A},-) \cong \operatorname{Ext}_{\mathbf{A}}^{*}(\mathbf{A},-)$. This generalizes the classical result: for any $k$-algebra $\Lambda, H^{*}(\Lambda,-) \cong \operatorname{Ext}_{\Lambda}^{*}(\Lambda,-)$. In distinction from the case of a single algebra, $H^{2}(\mathbf{A}, \mathbf{A})$ is neither the module of infinitesimal deformations nor that of "singular extensions" of $\mathbf{A}$ by $\mathbf{A}$. Rather, both of these are classified by the second cohomology group of another cohomology theory, $H_{s}^{*}(\mathbf{A},-)$, and there is a natural morphism $H_{s}^{*}(\mathbf{A},-) \rightarrow H^{*}(\mathbf{A},-)$. This is rarely an isomorphism; however, in the commutative case it is always a monomorphism.

We construct a functor ! from diagrams to ALG with some surprising features; denote the image of $\mathbf{A}$ by $\mathbf{A}$ !. For each $\mathbf{A}$ there is a full, exact embedding, also designated !, A-MOD $\rightarrow$ A!-MOD (the latter being the category of A!-bimodules). Hence, there is a natural transformation of graded bifunctors $\omega^{*}$ : $\operatorname{Ext}_{\mathbf{A}}^{*}(-,-) \rightarrow$ $\operatorname{Ext}_{\mathbf{A}}^{*}(-!,-!)$. The CCT now asserts:

For a wide class of diagrams, $\omega^{*}$ is an isomorphism. Among these are finite diagrams and those in which $k$ is noetherian and the $\mathbf{A}^{i}$ are the coordinate rings of the basic opens of $\operatorname{spec} k$. 
When $\omega^{*}$ is an isomorphism we then have $H^{*}(\mathbf{A}, \mathbf{A}) \cong H^{*}(\mathbf{A} !, \mathbf{A} !)$. This is not enough to force the deformation theories of $\mathbf{A}$ and $\mathbf{A}$ ! to coincide. First, a nontrivial deformation of $\mathbf{A}$ may induce the trivial deformation of $\mathbf{A}$ !; when this happens the infinitesimal must lie in the kernel of $H_{s}^{2}(\mathbf{A}, \mathbf{A}) \rightarrow H^{2}(\mathbf{A}, \mathbf{A})$. Second, if $I$ does not have a largest element there may be deformations of $\mathbf{A}$ ! which are not induced by deformations of $\mathbf{A}$. We remedy this problem for finite diagrams as follows: define $\# I=I \cup\{\infty\}$ and, to any diagram $\mathbf{A}$ over $I$, associate a diagram \# $\mathbf{A}$ over $\# I$. Then the deformation theories of $\mathbf{A}$ and $\# \mathbf{A}$ are identical, while every deformation of $(\# \mathbf{A})$ ! is induced by one of \#A. Hence the deformation theory of the algebra $(\# \mathbf{A})$ ! is a "snapshot" of that of $\mathbf{A}$. In the commutative case the two theories are identical; this is also true when $\mathbf{A}$ consists of a single morphism $\varphi: B \rightarrow A$. (We do not know if the same is true whenever $\omega^{*}$ is an isomorphism.) To obtain this equivalence we exhibit an explicit cochain map $C^{*}(\mathbf{A},-) \rightarrow C^{*}(\mathbf{A} !,-!)$ such that the morphism induced on the Hochschild cohomology is $\omega^{*}$.

Now $H^{*}(\mathbf{A}$ !, $\mathbf{A}$ !) has a rich structure $[\mathbf{G 1}]$ : it has a cup product in which it is associative and graded commutative, a graded Lie product under which its elements act as graded derivations of the cup product, and every infinitesimal deformation $\eta \in H^{2}\left(\mathbf{A}\right.$ !, $\mathbf{A}$ !) has an obstruction obs $\eta \in H^{3}(\mathbf{A}$ !, $\mathbf{A}$ !). All of this structure is, by virtue of the CCT, transferable to $H^{*}(\mathbf{A}, \mathbf{A})$. We do this explicitly in the simplest nontrivial case, namely when $\mathbf{A}$ consists of a single morphism $\varphi: B \rightarrow A$. Nijenhuis and Richardson have considered a restricted concept of deformation in this case; namely, they require that $\varphi$ be a monomorphism and that $A$ remain fixed [NR]. In particular, we set Nijenhuis' beautiful, but perplexing, paper [N] in a context which removes the mystery concerning the provenance of his graded Lie structure.

Building on the Harrison cohomology [Ha] of a commutative algebra, we describe natural "commutative" cohomologies $\operatorname{Har}^{*}(\mathbf{A},-)$ and $\operatorname{Har}_{s}^{*}(\mathbf{A},-)$ in the commutative case. Then, as before, $\operatorname{Har}_{s}^{2}(\mathbf{A}, \mathbf{A})$ is the module of infinitesimal deformations of $\mathbf{A}$ to diagrams of commutative algebras. This is most important, of course, when $\mathbf{A}$ is obtained from a complex manifold or a smooth algebraic variety $\mathcal{X}$. If $I$ is a covering of $\mathcal{X}$ by acyclic neighborhoods then $\operatorname{Har}_{s}^{2}(\mathbf{A}, \mathbf{A})$ is precisely the group of infinitesimal deformations of $\mathcal{X}$ and, so, is intrinsic to $\mathcal{X}$. Probably all elements of $H_{s}^{2}(\mathbf{A}, \mathbf{A})$ should be viewed as infinitesimal deformations of $\mathcal{X}$ to possibly "noncommutative" varieties, whatever this might mean. As an example of what it should mean, we deform a diagram of commutative algebras representing $P^{2}(k)$ to a diagram of noncommutative algebras. (Here we must assume $k$ is a field.) We show that the construction extends to any variety with a dominant morphism to $P^{2}(k)$. The resulting objects still have "local rings" which, however, are not commutative. When the characteristic of $k$ is 0 , this is formal since we do not discuss the convergence of any of the power series involved; but when the characteristic is positive, the series in this example are actually polynomials.

To have an analog of the CCT for the Harrison complexes would require extending Harrison's definitions to certain noncommutative rings, since $\mathbf{A}$ ! is virtually never commutative. Such an extension exists but is outside the scope of this paper. (André has defined a cohomology theory for commutative algebras which 
behaves well with respect to localization $[\mathbf{A}]$. Schlessinger and Stasheff have recently announced that it is identical to Harrison's cohomology.)

The results presented here comprise the first step in algebraizing the analytic deformation theory. The ultimate goal is, of course, to transfer back to manifolds or schemes information obtained purely algebraically. For example, a morphism of complex manifolds, $f: \mathcal{X} \rightarrow \mathcal{Y}$, can be represented as a diagram $\mathbf{A}$ of commutative algebras. Let $I_{2}$ and $I_{1}$ be coverings of $\mathcal{X}$ and $\mathscr{Y}$ by acyclic coordinate neighborhoods. Partially order $I=I_{2} \cup I_{1}$ as follows: $U \leqslant V$ if and only if $U \subseteq V$ (in $\mathcal{X}$ or P) ) or $f U \subseteq V$ (in $\mathcal{Y}$ ). Then $\mathbf{A}$ will assign to each open set the algebra of holomorphic functions defined on it. If $U \leqslant V$ the corresponding morphism of $\mathbf{A}$ will be either restriction or composition with $f$. It is natural to conjecture that: (1) Both $H^{*}(\mathbf{A}, \mathbf{A})$

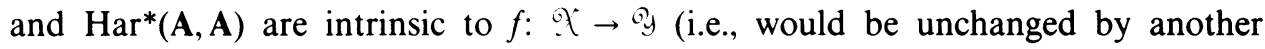
choice of acyclic covers); and (2) $\operatorname{Har}_{s}^{2}(\mathbf{A}, \mathbf{A})$ is the group of infinitesimal deformations of $f$, where $\mathscr{X}, \mathscr{P}$, and $f$ are all allowed to change simultaneously. The algebraic theory thus suggests that to $f$, as to $x$, there are associated in a natural way certain cohomology groups with graded Lie structures.

Deformation theory has a long history and a large literature. The analytic theory rests on the fundamental work of Froehlicher and Nijenhuis [FN] and Kodaira and Spencer [KS1, KS2], and has been enriched by scores of later major contributions, most of them referenced in the outstanding bibliography of Sundararaman [Su]. The idea of deforming a morphism of complex manifolds (in the special case where the target is fixed) appears in Kodaira [K]. For the algebraic theory one has [G1-G6], of which [G1, G2] suffice to understand this paper. ([G5] is an earlier attempt to algebraize the analytic theory.) In addition there are basic papers by Lichtenbaum and Schlessinger [LS], Rim [Rm], and Schlessinger [Scl], amongst many others. The reader should be aware that it is possible (perhaps essential) to define algebra deformations more generally than by power series (cf. $[\mathbf{R m}])$. The latter, however, very efficiently suggest what the problems and theorems should be, so we make no apology for our present parochial approach.

Some of the results of this paper appear in the dissertation [Sch] of the second author, to whom, in particular, is due the concept of a module over a diagram, as well as the definition of the ring whose cohomology and deformation theory coincide with that of a diagram. The second author wishes to express his gratitude to P. J. Freyd, M. Gerstenhaber, and S. S. Shatz for their advice and guidance, both mathematical and otherwise.

1. Modules over diagrams. Suppose that we have a diagram A: $I \rightarrow$ ALG. What we denote by $\mathbf{A}^{i}$ is properly $\mathbf{A}(i)$ and, for $i \leqslant j, \varphi^{i j}: \mathbf{A}^{j} \rightarrow \mathbf{A}^{i}$ is $\mathbf{A}(i \rightarrow j)$.

A left module $\mathbf{M}$ over $\mathbf{A}$ is a contravariant functor from the same index set $I$ to the category of abelian groups with the property that

(i) $\mathbf{M}(i)=\mathbf{M}^{i}$ is a left module over $\mathbf{A}^{i}$ for all $i$; and

(ii) for each $i \leqslant j, \mathbf{M}(i \rightarrow j)=T^{i j}: \mathbf{M}^{j} \rightarrow \mathbf{M}^{i}$ is a left $\mathbf{A}^{j}$-module morphism when $\mathbf{M}^{i}$ is viewed as a left $\mathbf{A}^{j}$-module by virtue of the morphism $\varphi^{i j}: \mathbf{A}^{j} \rightarrow \mathbf{A}^{i}$, as we shall do throughout without further comment.

Right modules and bimodules are defined similarly. Every diagram of algebras is a bimodule over itself. When every $\mathbf{A}^{i}$ is commutative we define a symmetric $\mathbf{A}$-module 
to be an A-bimodule $\mathbf{M}$ in which each $\mathbf{M}^{i}$ is a symmetric $\mathbf{A}^{i}$-module. The category of symmetric A-modules is clearly isomorphic to the category of left A-modules. When we make statements concerning symmetric A-modules we shall say we are in the commutative case.

A natural transformation $f: \mathbf{M} \rightarrow \mathbf{N}$ will be a morphism if, for all $i, f^{i}=f(i)$ : $\mathbf{M}^{i} \rightarrow \mathbf{N}^{i}$ is an $\mathbf{A}^{i}$-module morphism. The category of $\mathbf{A}$-bimodules is denoted A-MOD. It is abelian and bicomplete, i.e. contains arbitrary products and coproducts. (All constructions are made "pointwise.")

Let $\mathbf{A}^{i e}=\mathbf{A}^{i} \otimes\left(\mathbf{A}^{i}\right)^{\text {op }}$, the enveloping algebra of $\mathbf{A}^{i}$. (Recall that $a \otimes b \cdot a^{\prime} \otimes b^{\prime}=$ $a a^{\prime} \otimes b^{\prime} b$.) The category of left $\mathbf{A}^{i e}$-modules is isomorphic to $\mathbf{A}^{i}$-MOD. (The operation of $a \otimes b$ on an element $m$ of an $A^{i}$-bimodule $M$ is $a \otimes b \cdot m=a m b$.)

Let $P_{0}^{j}$ now be an $\mathbf{A}^{j}$-projective bimodule for each $j$. We can define a projective $\mathbf{P}$ by

$$
\mathbf{P}^{i}=\coprod_{j \geqslant i} \mathbf{A}^{i e} \otimes_{\mathbf{A}^{j e}} P_{0}^{j}
$$

If $i \geqslant h$ then $\mathbf{P}^{i} \rightarrow \mathbf{P}^{h}$ is given by tensoring with $\mathbf{A}^{h e}$ over $\mathbf{A}^{i e}$ (i.e. it is induced by $\left.\varphi^{h i} \otimes \varphi^{h i}\right)$. Now suppose that $\mathbf{P} \rightarrow \mathbf{N}$ is any morphism, while $\mathbf{M} \rightarrow \mathbf{N}$ is an epimorphism. For each $i$, choose an arbitrary lifting $f_{0}^{i}: P_{0}^{i} \rightarrow \mathbf{M}^{i}$ of $P_{0}^{i} \rightarrow \mathbf{P}^{i} \rightarrow \mathbf{N}^{i}$. Define $f^{i}: \mathbf{P}^{i} \rightarrow \mathbf{M}^{i}$ as

$$
f^{i}=\coprod_{j \geqslant i} T^{i j} f_{0}^{j}
$$

(i.e. $\left.f^{i}\left(a_{1}^{i} \otimes a_{2}^{i} \otimes p^{j}\right)=a_{1}^{i} \otimes a_{2}^{i} \cdot T^{i j} f_{0}^{j}\left(p^{j}\right)=a_{1}^{i} T^{i j} f_{0}^{j}\left(p^{j}\right) a_{2}^{i}\right)$. Then the $f^{i}$ determine a $\mathbf{P} \rightarrow \mathbf{M}$ which lifts $\mathbf{P} \rightarrow \mathbf{N}$, and we see that $\mathbf{P}$ is projective. Note that, since $\mathbf{A}^{j}$-MOD has enough projectives, A-MOD does as well. By a $j$-primitive projective we shall mean a projective $\mathbf{P}$ defined by (1), with $P_{0}^{i}=0$ when $i \neq j$. Then A-MOD has enough projectives which are coproducts of primitives.

When $I$ is noetherian (defined below), these are the only projectives, as we now show. Observe that a left adjoint to an exact functor preserves projectives. (Dually, a right adjoint to an exact functor preserves injectives.) Now, for each $i$, there is a full, exact embedding functor, $F^{i}: \mathbf{A}^{i}$-MOD $\rightarrow$ A-MOD. $\left[F^{i}(M)^{j}=0\right.$ if $j \neq i$ and $F^{i}(M)^{i}$ $=M$.] Define $L^{i}$ and $R^{i}: \mathbf{A}-\mathrm{MOD} \rightarrow \mathrm{A}^{i}$-MOD as follows:

a. $L^{i}(M)=\mathbf{M}^{i} / \cup_{j>i} T^{i j}\left(\mathbf{M}^{j}\right)$, where $\cup_{j>i} T^{i j}\left(\mathbf{M}^{j}\right)$ designates the $\mathbf{A}^{i}$-submodule generated by the $T^{i j}\left(\mathbf{M}^{j}\right)$; if $i \in I$ is maximal, set $L^{i}(\mathbf{M})=\mathbf{M}^{i}$.

b. $R^{i}(\mathbf{M})=\bigcap_{j<i} \operatorname{ker} T^{j i}$; if $i \in I$ is minimal, set $R^{i}(\mathbf{M})=\mathbf{M}^{i}$.

It is easy to see that these are left and right adjoints, respectively, to $F^{i}$.

If $\mathbf{P}$ is projective in $\mathbf{A}-\mathrm{MOD}$ then $L^{i} \mathbf{P}$ is projective in $\mathbf{A}^{i}$-MOD and $\mathbf{P}^{i}=L^{i} \mathbf{P} \oplus$ $\cup_{j<i} T^{i j}\left(\mathbf{P}^{j}\right)$. Define $\mathbf{P}^{\prime}$ by (1) with $P_{0}^{j}=L^{j} \mathbf{P}$. For each $j \geqslant i, T^{i j}: \mathbf{P}^{j} \rightarrow \mathbf{P}^{i}$, when restricted to $L^{j} \mathbf{P}$, gives rise to an $\mathbf{A}^{i}$-morphism $\mathbf{A}^{i e} \otimes L^{j} \mathbf{P} \rightarrow \mathbf{P}^{i}$ which we also denote $T^{i j}$. Define $g^{i}: \mathbf{P}^{i} \rightarrow \mathbf{P}^{i}$ as $g^{i}=\amalg_{j \geqslant i} T^{i j}$. Note that $g^{i}$ is the identity when restricted to $L^{i} \mathbf{P}$; so, written as a matrix,

$$
g^{i}=\left(\begin{array}{cc}
\mathrm{id} & 0 \\
0 & g_{1}^{i}
\end{array}\right) \quad \text { where } g_{1}^{i}=\coprod_{j>i} T^{i j}
$$


The various $g^{i}$ unite to form a morphism $g: \mathbf{P}^{\prime} \rightarrow \mathbf{P}$, and $L^{i}(g): L^{i} \mathbf{P}^{\prime}=L^{i} \mathbf{P} \rightarrow L^{i} \mathbf{P}$ is the identity.

A poset $I$ is noetherian if every strictly ascending chain is finite. Suppose that we have such an $I$. If $i$ is maximal in $I$ then $g^{i}$ is the identity and, therefore, is an epimorphism. Next, let $i$ be maximal in $\left\{j \mid g^{j}\right.$ is not an epimorphism $\}$ and let $a^{i}$ be in $T^{i j}\left(\mathbf{P}^{j}\right)$ for some $j>i$; say $a^{i}=T^{i j}\left(a^{j}\right)$. Now, there is a $p \in \mathbf{P}^{j}$ with $a^{j}=g^{j}(p)$, so $a^{i}=T^{i j} g^{j}(p)$. Consequently, $1 \otimes 1 \otimes p \in \mathbf{A}^{i e} \otimes \mathbf{P}^{j}$ satisfies $g^{i}(1 \otimes 1 \otimes p)=a^{i}$. It follows easily that $g^{i}$ is an epimorphism; hence, $g$ is as well.

Since $\mathbf{P}$ is projective there is a splitting $f: \mathbf{P} \rightarrow \mathbf{P}^{\prime}$ and

$$
f^{i}=\left(\begin{array}{cc}
\mathrm{id} & 0 \\
0 & f_{1}^{i}
\end{array}\right) \quad \text { where } g_{1}^{i} f_{1}^{i}=\mathrm{id}
$$

So $\mathbf{P}^{\prime}=\mathbf{P} \oplus \mathbf{P}^{\prime \prime}$ and $\mathbf{P}^{\prime \prime}$ is projective. Since $L^{i}$, as a left adjoint, preserves coproducts and $L^{i}(f)$ is the identity, it is immediate that $L^{i} \mathbf{P}^{\prime \prime}=0$. We claim that $\mathbf{P}^{\prime \prime}=0$. If $i$ is maximal, then $\mathbf{P}^{\prime \prime}=L^{i} \mathbf{P}^{\prime \prime}=0$. Assume, inductively, that $\mathbf{P}^{\prime \prime} j=0$ for $j>i$. Then $\mathbf{P}^{\prime \prime}=L^{i} \mathbf{P}^{\prime \prime} \oplus \cup_{j>i} T^{i j}\left(\mathbf{P}^{\prime \prime} j\right)=0$. Hence, $\mathbf{P}^{\prime \prime}=0$ and $\mathbf{P}=\mathbf{P}^{\prime}$. We have shown: if $I$ is noetherian, then every projective in A-MOD has the form (1) with $P_{0}^{j}=L^{j} \mathbf{P}$.

An A-bimodule $\mathbf{M}$ is finitely generated if $\mathbf{M}^{i}$ is a finitely generated $\mathbf{A}^{i}$-bimodule for all $i$. The coproduct gives the set of isomorphism classes of finitely generated projectives the structure of an abelian group, which we denote $K_{0}(\mathbf{A})$ [Ba1, VIII.1 and IX.1]. For each $p \in I$, let $I_{p}=\{i \in I \mid i \geqslant p\}$ be the filter defined by $p$. If $I_{p}$ is finite for all $p$, then $I$ is noetherian, and the above computation of projectives immediately implies $K_{0}(\mathbf{A})=\prod_{I} K_{0}\left(\mathbf{A}^{i}\right)$. The description of $K_{0}(\mathbf{A})$ for arbitrary (noetherian) $\mathbf{A}$ is more complicated.

Finally, we note that the above discussion of projectives can be dualized, yielding: Among the injectives in A-MOD are those modules I given by

$$
\mathbf{I}^{i}=\prod_{j \leqslant i} I_{0}^{j}, \quad \text { where } I_{0}^{j} \text { is an arbitrary injective in } \mathbf{A}^{j} \text {-MOD. }
$$

(Of course, $I_{0}^{j}=R^{j} \mathbf{I}$.) If $h \leqslant i$, then $\mathbf{I}^{i} \rightarrow \mathbf{I}^{h}$ is simply the projection. Moreover, since $\mathbf{A}^{i}$-MOD has enough injectives, A-MOD does as well. An injective $\mathbf{I}$ is $j$-primitive if it is given by (2) with $I_{0}^{i}=0$ for $i \neq j$. Then A-MOD has enough injectives which are products of primitives. If the index set is artinian then every injective has the form (2).

The simplest diagram, $\varphi: B \rightarrow A$, will, by abuse of language, be called "the diagram $\varphi$," and, if $T: N \rightarrow M$ is a module over it, we will likewise speak of "the module $T$."

2. Deformations. For simplicity, we use power series to define deformations, as in [G2]: Let $A$ be a $k$-algebra and denote by $A[[t]]$ the ring of all $\sum_{i=0}^{\infty} a_{i} t^{i}$ with $a_{i} \in A$. This is an algebra over $k[[t]]$. Denote the latter by $k_{t}$. If the multiplication in $A$ is given by $\alpha: A \times A \rightarrow A$, then a deformation of $A$ is a $k_{t}$-algebra $A_{t}$ which, as a module, is just $A[[t]]$ but with a multiplication of the form $\alpha_{t}=\alpha+t \alpha_{1}+t^{2} \alpha_{2}$ $+\cdots$. Each $\alpha_{i}: A \times A \rightarrow A$ is a $k$-bilinear map extended to be a $k_{t}$-bilinear map 
$A_{t} \times A_{t} \rightarrow A_{t}$ [G2]. (If $A$ is unital then so is $A_{t}$; cf. §20.) A deformation of a morphism $\varphi: B \rightarrow A$ is a $k_{t}$-algebra morphism $\varphi_{t}: B_{t} \rightarrow A_{t}$, where $B_{t}$ and $A_{t}$ are deformations of $B$ and $A$, respectively, and $\varphi_{t}$ has the form $\varphi+t \varphi_{1}+t^{2} \varphi_{2}+\cdots$. Each $\varphi_{i}$ is a $k$-linear map $B \rightarrow A$ extended to be a $k_{t}$-linear map $B_{t} \rightarrow A_{t}$. A deformation of a diagram $\mathbf{A}$ is a diagram $\mathbf{A}_{t}$ of $k_{t}$-algebras, $\left\{\mathbf{A}_{t}^{i}, \varphi_{t}^{i j}\right\}$, where each $\mathbf{A}_{t}^{i}$ is a deformation of $\mathbf{A}^{i}$ and $\varphi_{t}^{i j}: \mathbf{A}_{t}^{j} \rightarrow \mathbf{A}_{t}^{i}$ is a deformation of $\varphi^{i j}$. (If some $\varphi^{i j}$ is unital then so is $\varphi_{t}^{i j}$; $\mathrm{cf}$. $\S 20$.)

Since any linear map between power series modules is given by a power series, a deformation of a diagram, $\mathbf{A}: I \rightarrow$ ALG, is just a factorization $\mathbf{A}_{t}$ of $\mathbf{A}$ through the category of deformed algebras. Two deformations $\mathbf{A}_{t}$ and $\mathbf{A}_{t}^{\prime}$ are equivalent if there is a natural transformation $F_{t}: \mathbf{A}_{t} \rightarrow \mathbf{A}_{t}^{\prime}$ which reduces to the identity modulo $t$. Thus, there are algebra morphisms $F_{t}{ }^{i}: \mathbf{A}_{t}^{i} \rightarrow \mathbf{A}_{t}^{\prime i}$ satisfying: if $i \leqslant j$ then $F_{t}^{i} \varphi_{t}^{i j}=\varphi_{t}^{\prime i j} F_{t}^{j}$. Each $F_{t}^{i}$ has the form $F_{t}^{i}=\mathrm{id}+t F_{1}^{i}+t^{2} F_{2}^{i}+\cdots$, where each $F_{m}^{i}$ is a $k$-linear map $\mathbf{A}^{i} \rightarrow \mathbf{A}^{\prime i}$ extended to be a $k_{t}$-linear map $\mathbf{A}_{t}^{i} \rightarrow \mathbf{A}_{t}^{\prime i}$ (these being identical as $k_{t}$-modules). A deformation is trivial if it is equivalent to the "null deformation," in which every algebra $\mathbf{A}^{i}$ is replaced by $\mathbf{A}^{i}[[t]]$ with its usual multiplication and each $\varphi_{t}^{i j}$ : $\mathbf{A}^{j}[[t]] \rightarrow \mathbf{A}^{i}[[t]]$ is just the extension of the corresponding $\varphi^{i j}: \mathbf{A}^{j} \rightarrow \mathbf{A}^{i}$. A diagram is rigid if every deformation is trivial. As in [G6], reducing modulo $t^{n}$ permits us to define deformations of order $n-1$. A deformation is null to order $n-1$ if it coincides with the null deformation modulo $t^{n}$ and is trivial to order $n-1$ if it is equivalent to one which is null to order $n-1$. One which is trivial to order $n-1$ for every $n$ is called quasitrivial, following [G6], where it was shown, in particular, that for a single algebra $A$ over a field of characteristic zero, a quasitrivial deformation is trivial. The CCT will enable us to transfer this and other results for algebras to the case of diagrams over a wide class of posets. Finally, $\mathbf{A}_{t}$ and $\mathbf{A}_{t}^{\prime}$ are weakly equivalent if there are elements $a_{t}^{i j} \in \mathbf{A}^{i}[[t]](i<j)$, with $a_{0}^{i j}=e^{i}$ (the unit of $\mathbf{A}^{i}$ ), satisfying

$$
\alpha_{t}^{i}\left(\varphi_{t}^{\prime i j}\left(a^{j}\right), a_{t}^{i j}\right)=\alpha_{t}^{i}\left(a_{t}^{i j}, \varphi_{t}^{i j}\left(a^{j}\right)\right) \text { for all } a^{j} \in \mathbf{A}^{j} .
$$

(The deformations are "within an inner automorphism" of being equivalent.) We call a deformation inessential if it is weakly equivalent to the null deformation, and we define inessential to order $n-1$ as above. In the commutative case there are no (nontrivial) inessential deformations.

Let $p: I \rightarrow J$ be a functor between posets (i.e., an order-preserving function). For any category $Q$ there is an induced functor $p^{*}: Q^{J^{\text {op }}} \rightarrow \mathbb{Q}^{I^{\text {op }}}$ (the functor categories). When $\mathbb{Q}=\mathrm{ALG}, p^{*}$ carries a diagram $\mathbf{A}$ over $J$ to the diagram over $I$ given by $\left(p^{*} \mathbf{A}\right)^{i}=A^{p(i)}$. When $\mathbb{Q}$ is the category of deformed algebras, $p^{*}$ carries a deformation of $\mathbf{A}$ to one of $p^{*} \mathbf{A}$; as a functor it preserves equivalence. Hence the deformation theory of A: $J^{\text {op }} \rightarrow$ ALG maps to that of $p^{*} \mathbf{A}: I^{\text {op }} \rightarrow$ ALG. This is not generally an embedding; however, in one case of special interest it is. We sharpen a poset $I$ by adjoining a largest element: $\# I=I \cup\{\infty\}$. Taking $\mathcal{Q}=\mathrm{ALG}$ and $p=$ the inclusion $I \rightarrow \# I$, we find that $p^{*}$ has a section $\#: \mathrm{ALG}^{I^{\text {op }}} \rightarrow \mathrm{ALG}^{(\# I)^{\text {op }}}$ given by $(\# \mathbf{A})^{\infty}=k$ and $(\# \mathbf{A})^{\infty} \rightarrow(\# \mathbf{A})^{i}=k \rightarrow \mathbf{A}^{i}$, the structure map of $\mathbf{A}^{i}$. Then the deformation theory of \#A clearly embeds in that of $\mathbf{A}$. In $\$ 20$ we shall prove these theories isomorphic by showing that the algebras and morphisms in a deformation of $\mathbf{A}$ are unital. 
3. The case of a single morphism: infinitesimal deformations. When the diagram $\mathbf{A}$ consists of a single morphism $\varphi: B \rightarrow A$, the appropriate complex is a mapping cylinder.

If we have a morphism between two cochain complexes,

$$
\begin{array}{cccccccc}
X: & \cdots & \rightarrow & X^{n} & \stackrel{\delta_{X}^{n}}{\rightarrow} & X^{n+1} & \rightarrow & \cdots \\
f \downarrow & & & f^{n} \downarrow & & \downarrow & & \\
Y: & \cdots & \rightarrow & Y^{n} & \stackrel{\delta_{Y}^{n}}{\rightarrow} & Y^{n+1} & \rightarrow & \cdots
\end{array}
$$

then $Z^{*}=X^{*} \oplus Y^{*-1}$ (the elements of which we consider as column vectors of length 2 ) is a complex with coboundary operator

$$
\delta^{n}=\left(\begin{array}{cc}
\delta_{X}^{n} & 0 \\
f^{n} & -\delta_{Y}^{n}
\end{array}\right)
$$

(cf. [Ba2]). Explicitly, $\delta^{n}(x, y)=\left(\delta_{X}^{n} x, f^{n} x-\delta_{Y}^{n-1} y\right)$. This $Z^{*}$ is the mapping cylinder of $f$. There is an obvious short exact sequence of complexes, $0 \rightarrow Y^{*-1} \rightarrow Z^{*}$ $\rightarrow X^{*} \rightarrow 0$, inducing a long exact sequence in the cohomology

$$
\cdots \rightarrow H^{n-1}\left(Y^{*}\right) \rightarrow H^{n}\left(Z^{*}\right) \rightarrow H^{n}\left(X^{*}\right) \rightarrow H^{n}\left(Y^{*}\right) \rightarrow \cdots .
$$

Now suppose that we have a module $T: N \rightarrow M$ over the morphism $\varphi: B \rightarrow A$. Take $X^{*}=C^{*}(B, N) \oplus C^{*}(A, M)$ and $Y^{*}=C^{*}(B, M)$, where in the latter $M$ is viewed as a $B$-bimodule by virtue of $\varphi$. Suppose that $\Gamma^{B} \in C^{n}(B, M)$ and $\Gamma^{A} \in$ $C^{n}(A, M)$. To conform with later notation denote an element of $C^{n-1}(B, M)$ by $\Gamma^{A B}$. Define $f^{n}: X^{n} \rightarrow Y^{n}$ by $f^{n}\left(\Gamma^{B}, \Gamma^{A}\right)=T_{*} \Gamma^{B}-\varphi^{*} \Gamma^{A}$, where $T_{*}: C^{n}(B, N) \rightarrow$ $C^{n}(B, M)$ and $\varphi^{*}: C^{n}(A, M) \rightarrow C^{n}(B, M)$ are the morphisms induced by $T$ and $\varphi$, respectively. To simplify notation, we write $T \Gamma^{B}$ for $T_{*} \Gamma^{B}$ and $\Gamma^{A} \varphi$ for $\varphi^{*} \Gamma^{A}$, the latter reflecting the contravariance of $\varphi^{*}$. We also denote $T$ by $T^{A B}$ and $\varphi$ by $\varphi^{A B}$, so that $f\left(\Gamma^{B}, \Gamma^{A}\right)=T^{A B} \Gamma^{B}-\Gamma^{A} \varphi^{A B}$, which will conform with the notation for the general diagram. The resulting mapping cylinder with $Z^{n}=C^{n}(B, N) \oplus C^{n}(A, M)$ $\oplus C^{n-1}(B, M)$ will be denoted by $C^{*}(\varphi, T)$ and its cohomology by $H^{*}(\varphi, T)$. One has

$$
\delta\left(\Gamma^{B}, \Gamma^{A} ; \Gamma^{A B}\right)=\left(\delta \Gamma^{B}, \delta \Gamma^{A} ; T^{A B} \Gamma^{B}-\Gamma^{A} \varphi^{A B}-\delta \Gamma^{A B}\right) .
$$

Taking for $T$ the morphism $\varphi$ itself, we claim that $H^{2}(\varphi, \varphi)$ is, in a natural way, the module of infinitesimal deformations of $\varphi$.

For suppose that we have a deformation $\varphi_{t}: B_{t} \rightarrow A_{t}$ of $\varphi$. Let $\alpha_{t}=\alpha_{0}+t \alpha_{1}+$ $t^{2} \alpha_{2}+\cdots$ be the multiplication in $A_{t}$ and $\beta_{t}=\beta_{0}+t \beta_{1}+t^{2} \beta_{2}+\cdots$ be that in $B_{t}$. Write $\varphi_{t}=\varphi_{0}+t \varphi_{1}+t^{2} \varphi_{2}+\cdots$. (Here $\varphi_{0}=\varphi$.) The triple of first-order terms, $\left(\beta_{1}, \alpha_{1} ; \varphi_{1}\right)$, is, we claim, an element of $Z^{2}(\varphi, \varphi)$, the group of 2-cocycles, and its cohomology class may be viewed as the infinitesimal of the deformation. That $\delta \beta_{1}=\delta \alpha_{1}=0$ is standard (cf. [G2]). However, we also have for all $b, b^{\prime} \in B$ that

$$
\alpha_{t}\left(\varphi_{t} b, \varphi_{t} b^{\prime}\right)=\varphi_{t} \beta_{t}\left(b, b^{\prime}\right)
$$

Comparing first-order terms, we have $\alpha_{1}\left(\varphi b, \varphi b^{\prime}\right)+\left(\varphi_{1} b\right) \varphi b^{\prime}+\varphi b\left(\varphi_{1} b^{\prime}\right)=\varphi_{1}\left(b b^{\prime}\right)$ $+\varphi \beta_{1}\left(b, b^{\prime}\right)$, or $\varphi \beta_{1}-\alpha_{1} \varphi-\delta \varphi_{1}=0$. So $\left(\alpha_{1}, \beta_{1} ; \varphi_{1}\right) \in Z^{2}(\varphi, \varphi)$. That only the cohomology class is important follows from 
LEMMA. Replacing $\varphi_{t}$ by an equivalent deformation changes $\left(\beta_{1}, \alpha_{1} ; \varphi_{1}\right)$ by a coboundary. Conversely, every cohomologous cocycle is the infinitesimal of an equivalent deformation.

Proof. Suppose that we have an equivalence of deformations

$\begin{array}{ccc}B_{t} & \stackrel{\varphi_{t}}{\rightarrow} & A_{t} \\ G_{t} \downarrow & & \downarrow F_{t} \\ B_{t}^{\prime} & \stackrel{\varphi_{t}^{\prime}}{\rightarrow} & A_{t}^{\prime}\end{array}$

where $B_{t}$ is the $B_{t}$-module $B[[t]]$ with multiplication $\beta_{t}=\beta+t \beta_{1}+\cdots$, and $B_{t}^{\prime}$ is the same module with multiplication $\beta_{t}^{\prime}=\beta+t \beta_{1}^{\prime}+\cdots$. (Here $\beta$ is the original multiplication in B.) Similarly, let $A_{t}$ have multiplication $\alpha_{t}=\alpha+t \alpha_{1}+\cdots$ and $A_{t}^{\prime}$ have multiplication $\alpha_{t}^{\prime}=\alpha+t \alpha_{1}^{\prime}+\cdots$. Then $\beta_{t}^{\prime}\left(b_{1}, b_{2}\right)=G_{t} \beta_{t}\left(G_{t}^{-1} b_{1}, G_{t}^{-1} b_{2}\right)$, $\alpha_{t}^{\prime}\left(a_{1}, a_{2}\right)=F_{t} \alpha_{t}\left(F_{t}^{-1} a_{1}, F_{t}^{-1} a_{2}\right)$, and $\varphi_{t}^{\prime}=F_{t} \varphi_{t} G_{t}^{-1}$. Writing $F_{t}=\mathrm{id}_{A}+t F_{1}+\cdots$, $G_{t}^{\prime}=\mathrm{id}_{B}+t G_{1}^{\prime}+\cdots$, and $\varphi_{t}^{\prime}=\varphi+t \varphi_{1}^{\prime}+\cdots$, the previous equations give

$$
\begin{aligned}
\beta_{1}^{\prime}\left(b_{1}, b_{2}\right) & =\beta_{1}\left(b_{1}, b_{2}\right)+G_{1}\left(b_{1} b_{2}\right)-\left(G_{1} b_{1}\right) b_{2}-b_{1} G_{1}\left(b_{2}\right) \\
& =\left(\beta_{1}-\delta G_{1}\right)\left(b_{1}, b_{2}\right) \\
\alpha_{1}^{\prime}= & \alpha_{1}-\delta F_{1}, \text { and } \varphi_{1}^{\prime}=\varphi_{1}-\left(\varphi G_{1}-F_{1} \varphi\right)
\end{aligned}
$$

Thus $\left(\beta_{1}^{\prime}, \alpha_{1}^{\prime} ; \varphi_{1}^{\prime}\right)=\left(\beta_{1}, \alpha_{1} ; \varphi_{1}\right)-\delta\left(G_{1}, F_{1} ; 0\right)$, and $\left(\beta_{1}^{\prime}, \alpha_{1}^{\prime} ; \varphi_{1}^{\prime}\right) \sim\left(\beta_{1}, \alpha_{1} ; \varphi_{1}\right)$. Now let $(G, F ; a)$ be an arbitrary element of $C^{1}(\varphi, \varphi)$. Here $a$ is simply an element of $A$, since $A=C^{0}(B, A)=C^{0}(A, A)$. But then $\delta(0,-\delta a ; a)=0$, and so $\delta(G, F+\delta a ; 0)$ $=\delta(G, F ; a)$. It is sufficient, therefore, to show that the cocycle $\left(\beta_{1}, \alpha_{1} ; \varphi_{1}\right)$ associated with the deformation $\varphi_{t}: B_{t} \rightarrow A_{t}$ can be replaced by one of the form $\left(\beta_{1}, \alpha_{1} ; \varphi_{1}\right)-\delta(G, F ; 0)$ by passing to an equivalent deformation. To this end, define $G_{t}: B[[t]] \rightarrow B[[t]]$ by $G_{t}(b)=b+t G(b)$, extended to be $k_{t}$-linear, and define $F_{t}$ similarly. Define a new multiplication $\beta_{t}$ on $B[[t]]$ by $\beta_{t}^{\prime}\left(b_{1}, b_{2}\right)=$ $G_{t} \beta_{t}\left(G_{t}^{-1} b_{1}, G_{t}^{-1} b_{2}\right)$. Define $\alpha_{t}^{\prime}$ similarly on $A[[t]]$ and set $\varphi_{t}^{\prime}=F_{t} \varphi_{t} G_{t}^{-1}$. This gives the required equivalent deformation.

Note in the proof that we showed that every 1-cochain is cohomologous to one of the form $(F, G, 0)$.

For a commutative $k$-algebra $A$ and a symmetric-module $M$, Harrison [Ha] has described an important subcomplex of $C^{*}(A, M)$. Following Barr, we denote that complex by $\mathrm{Ch}^{*}(A, M)$ and its cohomology by $\operatorname{Har}^{*}(A, M)$. To describe it we remind the reader that if $J$ and $K$ are totally ordered sets then a shuffle of $J$ through $K$ is a permutation $\pi \in S_{J \cup K}$ whose restrictions $J \rightarrow J \cup K$ and $K \rightarrow J \cup K$ are order preserving [Ha]. The shuffle product of $J$ and $K$ is the formal sum $J * K=\Sigma(-1)^{\pi} \pi$. If $f \in C^{n}(A, M)$ we interpret $f\left(\left\langle a_{1}, \ldots, a_{l}\right\rangle *\left\langle a_{l+1}, \ldots, a_{n}\right\rangle\right)$ as $\Sigma(-1)^{\pi} f\left(a_{\pi 1}, \ldots, a_{\pi n}\right)$. A Harrison cochain is a Hochschild cochain which vanishes on shuffle products. Thus $\operatorname{Ch}^{0}(A, M)$ and $\operatorname{Ch}^{1}(A, M)$ coincide with the usual Hochschild groups, while $\mathrm{Ch}^{2}(A, M)$ consists of the symmetric 2-cochains. The inclusion of cochain complexes induces $\operatorname{Har}^{*}(A, M) \rightarrow H^{*}(A, M)$. It is easy to check that $\operatorname{Har}^{2}(A, A)$ is the module of infinitesimal deformations of $A$ to commutative algebras. 
Let $\varphi: B \rightarrow A$ be a morphism of commutative algebras and $T: N \rightarrow M$ a left $\varphi$-module. Then $T$ may be regarded as a $\varphi$-bimodule and restriction of our earlier cochain map produces $\mathrm{Ch}^{*}(B, N) \oplus \mathrm{Ch}^{*}(A, M) \rightarrow \mathrm{Ch}^{*}(B, M)$. The resulting mapping cylinder and cohomology are denoted $\operatorname{Ch}^{*}(\varphi, T)$ and $\operatorname{Har}^{*}(\varphi, T)$. As in the case of an algebra, the inclusion $\operatorname{Ch}^{*}(\varphi, T) \rightarrow C^{*}(\varphi, T)$ induces $\operatorname{Har}^{*}(\varphi, T) \rightarrow$ $H^{*}(\varphi, T)$ and $\operatorname{Har}^{2}(\varphi, \varphi)$ is the module of infinitesimal deformations of $\varphi$ to morphisms of commutative algebras.

4. A single morphism: obstructions. Consider a single algebra $A$ and an $A$-bimodule $M$. If $f^{m} \in C^{m}(A, M)$ and $g^{n} \in C^{n}(A, A)$, then we define $f^{m} \bar{o}_{i} g^{n} \in$ $C^{m+n-1}(A, M)$ by

(1)

$$
f^{m} \bar{o}_{i} g^{n}\left(a_{1}, \ldots, a_{m+n-1}\right)=f^{m}\left(a_{1}, \ldots, a_{i-1}, g\left(a_{i}, \ldots, a_{i+n-1}\right), a_{i+n}, \ldots, a_{m+n-1}\right)
$$

[G1]. We also define the cup product, $f^{m} \smile g^{n} \in C^{m+n}(A, M)$, to be

$$
f^{m} \smile g^{n}\left(a_{1}, \ldots, a_{m+n}\right)=f\left(a_{1}, \ldots, a_{m}\right) g\left(a_{m+1}, \ldots, a_{m+n}\right) .
$$

Note that this definition, unlike that of $\bar{o}_{i}$, makes sense when $f^{m} \in C^{m}(A, A)$ and $g^{n} \in C^{n}(A, M)$. The composition product, which we henceforth denote by $\bar{o}$ (instead of $\circ$ in [G1]), is

$$
f^{m \bar{o}} g^{n}=\sum_{i=1}^{m}(-1)^{(i-1)(n-1)} f^{m} \overline{\mathrm{o}}_{i} g^{n} .
$$

When $M=A$ this is a (graded right) pre-Lie product on $C^{*}(A, A)[\mathbf{G 1}]$. That is,

$$
\begin{aligned}
\left(f^{m} \bar{\circ} g^{n}\right) \bar{\circ} h^{p}-(-1)^{(n-1)(p-1)}\left(f^{m} \bar{\circ} h^{\dot{p}}\right) \bar{\circ} g^{n} \\
\quad=f^{m} \bar{\circ}\left(g^{n \circ} h^{p}-(-1)^{(n-1)(p-1)} h^{p} \bar{\circ} g^{n}\right) .
\end{aligned}
$$

Also when $M=A$, set

$$
\left[f^{m}, g^{n}\right]=f^{m} \bar{\circ} g^{n}-(-1)^{(m-1)(n-1)} g^{n} \bar{\circ} f^{m} .
$$

This was shown in [G1] to define a graded Lie product on $C^{*}(A, A)$, provided that we consider the degree of a cochain to be one less than its dimension. The cocycles form a sub-Lie algebra in which the coboundaries form an ideal. Thus, there is a graded Lie algebra structure on $H^{*}(A, A)$. Similarly, the cup product is well defined on the cohomology. Indeed, it gives $H^{*}(A, M)$ a bimodule structure over $H^{*}(A, A)$. There are several basic identities connecting the Lie and cup product structures in $H^{*}(A, A)$. These and the proof that the latter is a graded commutative product appear in [G1].

Now, let $\alpha_{t}=\alpha_{0}+t \alpha_{1}+t^{2} \alpha_{2}+\cdots$ be a deformation of $A$ (where $\alpha_{0}$ is the original multiplication). Then, it was shown in [G2] that $\alpha_{1} \in Z^{2}(A, A)$ and $\alpha_{1}{ }^{\circ} \alpha_{1}$ $=\delta \alpha_{2}$. Thus, for any $\alpha_{1} \in Z^{2}$, we may view the cohomology class of $\alpha_{1} \bar{\circ} \alpha_{1}$ (which is always a 3-cocycle), as the primary obstruction to the extension of $\alpha_{1}$ to a deformation. Note, incidentally, that $\left[\alpha_{1}, \alpha_{1}\right]=2 \alpha_{1} \bar{\alpha} \alpha_{1}$. The analogous primary 
obstruction to an infinitesimal deformation of a morphism $\varphi: B \rightarrow A$ is obtained by examining the second order terms in (3.1). These give

$$
\begin{aligned}
\alpha_{2}\left(\varphi b, \varphi b^{\prime}\right)+\alpha_{1}\left(\varphi_{1} b, \varphi b^{\prime}\right)+ & \alpha_{1}\left(\varphi b, \varphi_{1} b^{\prime}\right)\left(\varphi_{1} b\right)\left(\varphi_{1} b^{\prime}\right) \\
& +\left(\varphi_{2} b\right)\left(\varphi b^{\prime}\right)+(\varphi b)\left(\varphi_{2} b^{\prime}\right) \\
= & \varphi_{2}\left(b b^{\prime}\right)+\varphi_{1} \beta_{1}\left(b, b^{\prime}\right)+\varphi \beta_{2}\left(b, b^{\prime}\right) .
\end{aligned}
$$

Now, if we have $\varphi^{A B}: B \rightarrow A$, an $A$-bimodule $M$, and $\Gamma^{A} \in C^{m}(A, M), \Gamma^{B} \in$ $C^{n}(B, A)$, then there is only one reasonable way to define $\Gamma^{A} \bar{o}_{i} \Gamma^{B} \in$ $C^{m+n-1}(B, M)$, namely by

$$
\begin{aligned}
\left(\Gamma^{A} \overline{\mathrm{o}}_{i} \Gamma^{B}\right) & \left(b_{1}, \ldots, b_{m+n-1}\right) \\
& =\Gamma^{A}\left(\varphi b_{1}, \ldots, \varphi b_{i-1}, \Gamma^{B}\left(b_{i}, \ldots, b_{i+n-1}\right), \varphi b_{i+n}, \ldots, \varphi b_{m+n-1}\right) .
\end{aligned}
$$

Setting

$$
\Gamma^{A} \bar{\circ} \Gamma^{B}=\sum_{i=1}^{n}(-1)^{(i-1)(n-1)} \Gamma^{A} \bar{o}_{i} \Gamma^{B}
$$

as before, and taking for $M$ just $A$ itself, (5) is identical with

$$
\alpha_{1}{ }^{\circ} \varphi_{1}-\varphi_{1} \beta_{1}+\varphi_{1}-\varphi_{1}=\varphi \beta_{2}-\alpha_{2} \varphi-\delta \varphi_{2} .
$$

Since $\beta_{1} \bar{\circ} \beta_{1}=\delta \beta_{2}$ and $\alpha_{1} \bar{o} \alpha_{1}=\delta \alpha_{2}$, we have

$$
\left(\beta_{1} \bar{\circ} \beta_{1}, \alpha_{1} \bar{\circ} \alpha_{1} ; \alpha_{1} \bar{\circ} \varphi_{1}-\varphi_{1} \bar{\circ} \beta_{1}+\varphi_{1}-\varphi_{1}\right)=\delta\left(\beta_{2}, \alpha_{2} ; \varphi_{2}\right) \text {. }
$$

One would like now to introduce a pre-Lie product on $C^{*}(\varphi, \varphi)$ with properties similar to that on $C^{*}(A, A)$. In view of $(8)$ it is natural to define

$$
C^{m}(\varphi, \varphi) \times C^{n}(\varphi, \varphi) \rightarrow C^{m+n-1}(\varphi, \varphi)
$$

by

$$
\text { (9) } \begin{aligned}
\left(\Gamma^{B}, \Gamma^{A} ; \Gamma^{A B}\right) \cdot\left(\Gamma^{B}, \Gamma^{\prime A} ; \Gamma^{A B}\right)^{n} \\
=\left(\Gamma^{B} \bar{\circ} \Gamma^{\prime B}, \Gamma^{A} \bar{\circ} \Gamma^{\prime A} ; \Gamma^{A} \bar{\circ} \Gamma^{A B}+(-1)^{n-1} \Gamma^{A B} \bar{\circ} \Gamma^{B}+\Gamma^{A B} \smile \Gamma^{A B}\right) .
\end{aligned}
$$

On the left we write "." instead of " $\circ$ " because this is not a pre-Lie product, and, indeed, it does not appear possible to give one. However, the left side of (8) is just the square of $\left(\beta_{1}, \alpha_{1} ; \varphi_{1}\right)$ in this product, since the degree is odd.

Note that (9) is meaningful if we have a module $T: N \rightarrow M$ over a morphism $\varphi: B \rightarrow A$ and $\left(\Gamma^{\prime B}, \Gamma^{\prime A} ; \Gamma^{A B}\right) \in C^{n}(\varphi, \varphi)$, while $\left(\Gamma^{B}, \Gamma^{A} ; \Gamma^{A B}\right) \in C^{m}(\varphi, T)$.

Our theory will show that: (i) the square of a cocyle of even dimension is again a cocycle, and the same is true in odd dimensions when the characteristic is 2; (ii) the graded commutator of two cocycles is again a cocycle, and is a coboundary if one is; and (iii) the induced multiplication in $H^{*}(\varphi, \varphi)$ makes the latter into a graded Lie algebra. These can all be proved by direct computation, but that seems to be very difficult, particularly (iii), which is unmanageable without a reformulation of the Jacobi identity as given in [Sch]. 
As in [G2], having passed the primary obstruction, there is another, and so on. We will not write these down since the CCT reduces all these questions to the corresponding classical ones in the deformation theory of a single ring. We remark only that the higher obstructions have no expression in terms of ".".

5. A single morphism: cup products. As before, suppose that we have a module $T^{A B}: N \rightarrow M$ over $\varphi^{A B}: B \rightarrow A$. If $\Gamma=\left(\Gamma^{B}, \Gamma^{A} ; \Gamma^{A B}\right) \in C^{m}(\varphi, \varphi)$ and $\Gamma^{\prime}=$ $\left(\Gamma^{B}, \Gamma^{\prime A} ; \Gamma^{A B}\right) \in C^{n}(\varphi, T)$, then we set

$$
\Gamma \smile \Gamma^{\prime}=\left(\Gamma^{B} \smile \Gamma^{\prime B}, \Gamma^{A} \smile \Gamma^{A} ; \Gamma^{A B} \smile T^{A B} \Gamma^{\prime B}+(-1)^{m} \Gamma^{A} \varphi^{A B} \smile \Gamma^{A B} .\right),
$$

and

$$
\Gamma^{\prime} \smile \Gamma=\left(\Gamma^{B} \smile \Gamma^{B}, \Gamma^{\prime A} \smile \Gamma^{A} ; \Gamma^{\prime A B} \smile \varphi^{A B} \Gamma^{B}+(-1)^{n} \Gamma^{\prime A} \varphi^{A B} \smile \Gamma^{A B}\right) .
$$

An easy calculation shows that this makes $C^{*}(\varphi, \varphi)$ into an associative ring and $C^{*}(\varphi, T)$ into a $C^{*}(\varphi, \varphi)$-bimodule. Moreover, one can show readily that $\delta\left(\Gamma \smile \Gamma^{\prime}\right)=\delta \Gamma \smile \Gamma^{\prime}+(-1)^{m} \Gamma \smile \delta \Gamma^{\prime}$, and similarly for $\delta\left(\Gamma^{\prime} \smile \Gamma\right)$. Thus $H^{*}(\varphi, \varphi)$ is an associative ring over which $H^{*}(\varphi, T)$ is a bimodule. Applying $\delta$ to (4.5) and evaluating as in [G1] shows that the multiplication is graded commutative, but this direct computation also may be avoided by the CCT.

In [G2] the first author considered one-parameter families of automorphisms of a single algebra $A$, showing that their infinitesimals were derivations and defining their obstructions. When the characteristic is $p>0$, these obstructions appear only at the $p, p^{2}, p^{3}, \ldots$ places; cf. [G4]. This, of course, also holds here (and for general diagrams) by virtue of the CCT. (One needs also $§ 18$.)

6. Allowable morphisms; relative projectives and injectives. Let $A$ be a $k$-algebra and let E: $0 \rightarrow M^{\prime} \rightarrow M \rightarrow M^{\prime \prime} \rightarrow 0$ be an exact sequence in $A$-MOD. The $k$-linearity requirement on cochains prevents $C^{n}(A, \mathbf{E})$ from being right exact for $n>0$. If, however, one restricts attention to those $\mathbf{E}$ which are $k$-split, then $C^{n}(A, \mathbf{E})$ is exact and induces the usual long exact cohomology exact sequence-making $H^{*}(A,-)$ a $\delta$-functor on $k$-split E. Let $|-|: A$-MOD $\rightarrow k$-MOD be the forgetful functor. $(|M|$ is $M$, considered only as a $k$-module.) Then the $|-|$-allowable $\mathbf{E}$ are those for which $|\mathrm{E}|$ is split. Consequently, one says that $H^{*}(A,-)$ is a $|-|$-relative $\delta$-functor. A morphism $f: M \rightarrow N$ is allowable if there is a splitting $\lambda:|N| \rightarrow|M|$ (i.e., $|f| \lambda|f|$ $=|f|)[\mathbf{M}$, Chapters IX, X]. We wish to extend these concepts to the case of a diagram A.

A morphism $f: \mathbf{M} \rightarrow \mathbf{N}$ will be allowable if and only if each $f^{i}: \mathbf{M}^{i} \rightarrow \mathbf{N}^{i}$ is. Note: we do not require the splittings for the various $f^{i}$ to commute with the "internal" morphisms of $\mathbf{M}$ and $\mathbf{N}$. An exact sequence in A-MOD is allowable if all its morphisms are allowable. Let $U: \mathbf{A}-\mathrm{MOD} \rightarrow k$-MOD be given by $U(\mathbf{M})=$ $\Pi_{I}\left|\mathbf{M}^{i}\right|$. Then observe that the allowable short exact sequences $E$ are those for which $U(E)$ is split. In the next section we shall define $H^{*}(\mathbf{A},-)$ and show it to be a $U$-relative $\delta$-functor. 
An A-bimodule $\mathbf{P}$ is a $U$-relative projective if it satisfies the lifting criterion for allowable epimorphisms. there are enough relative projectives in A-MOD if for every $\mathbf{M}$ there is a relative projective $\mathbf{P}$ and an allowable epimorphism $\mathbf{P} \rightarrow \mathbf{M} \rightarrow 0$. This will follow from the existence of a left adjoint to $U$ [M, Chapter IX]. Dually, one defines $U$-relative injective and establishes the presence of enough relative injectives by exhibiting a right adjoint to $U$.

Define $L, R: k$-MOD $\rightarrow$ A-MOD as follows:

(1) $L(V)^{i}=\amalg_{j \geqslant i} \mathrm{~A}^{i e} \otimes V$ and, for $j \geqslant i, L(V)^{j} \rightarrow L(V)^{i}$ is given by tensoring with $\mathbf{A}^{i e}$ over $\mathbf{A}^{j e}$.

(2) $R(V)^{i}=\amalg_{j \leqslant i} \operatorname{Hom}_{k}\left(\mathbf{A}^{j e}, V\right)$ and, for $j \geqslant i, R(V)^{j} \rightarrow R(V)^{i}$ is the projection. [Recall that $\operatorname{Hom}_{k}\left(\mathbf{A}^{j e}, V\right)$ is an $\mathbf{A}^{j}$-bimodule under the action $(a f b)(c \otimes d)=$ $f(b c \otimes d a)$. The morphism $\varphi^{i j}$ then gives it an $\mathbf{A}^{i}$-structure for any $i \geqslant j$.] An easy calculation shows that $L$ and $R$ are, respectively, left and right adjoints to $U$. (When $I=$ a point, $\mathbf{A}=A$, a single algebra, and $L$ and $R$ are the familiar adjoints to $|-|$ [M, pp. 266, 287].)

Alternatively, let $\mathbf{M}$ be an A-bimodule. For each $\mathbf{M}^{j}$, choose a $|-|$-relative projective cover $P_{0}^{j}$ and a $|-|$-relative injective extension $I_{0}^{j}$. Then define $\mathbf{P}$ and $\mathbf{I}$ by (1.1) and (1.2), respectively. These will be a $U$-relative projective cover and a $U$-relative injective extension.

Henceforth, we shall consider allowable morphisms exclusively; consequently, by "a morphism $\mathbf{M} \rightarrow \mathbf{N}$," we shall tacitly mean an allowable one.

7. The general diagram: Hochschild cohomology. We shall make use of the geometric realization functor to construct the Hochschild complex for a general diagram.

If $I$ is a partially ordered set then $\Sigma=\Sigma(I)$ is the simplicial complex whose simplices are the linearly ordered subsets of $I$; so

$$
\Sigma_{p}=p \text {-simplices }=\left\{\sigma=\left(i_{p}<\cdots<i_{1}<i_{0}\right)\right\} .
$$

Define the $r$ th-face of $\sigma \in \Sigma_{p}$ to be $\sigma_{r}=\left(i_{p}<\cdots<\hat{i}_{r}<\cdots<i_{0}\right)$, the $(p-1)$ simplex which results from the omission of $i_{r}$. The group of $p$-chains on $I$ (the free abelian group generated by $\left.\Sigma_{p}\right)$ is denoted $C_{p}(I)$ or $C_{p}$, and the boundary of $\sigma \in \Sigma_{p}$ is

$$
\partial \sigma=\sigma_{p}+\cdots+(-1)^{p-r} \sigma_{r}+\cdots+(-1)^{p} \sigma_{0} \in C_{p-1} .
$$

(Of course $\partial^{2}=0$.) We shall employ two functions $\Sigma \rightarrow \Sigma_{0}$, namely $v \sigma=i_{p}$ and $u \sigma=i_{0}$, where $\sigma=\left(i_{p}<\cdots<i_{0}\right)$. By convention, $(-1)^{c}=(-1)^{p}$ if $c \in C_{p}$.

Now suppose that we have an arbitrary diagram $\mathbf{A}=\left\{\mathbf{A}^{i}, \varphi^{i j}\right\}$ and an $\mathbf{A}$-bimodule $\mathbf{M}=\left\{\mathbf{M}^{i}, T^{i j}\right\}$. We define the $n$-cochains of $C^{*}(\mathbf{A}, \mathbf{M})$ to be

$$
C^{n}(\mathbf{A}, \mathbf{M})=\prod_{p \leqslant n} \prod_{\sigma \in \Sigma_{p}} C^{n-p}\left(A^{u \sigma}, M^{v \sigma}\right) .
$$

Thus an $n$-cochain $\Gamma$ is a set of Hochschild-cochains indexed by the simplices; we write $\Gamma=\left\{\Gamma^{\sigma}\right\}$. (When $\Gamma$ is an $n$-cochain and $\sigma$ is a $p$-simplex for $p>n$ we shall 
interpret $\Gamma^{\sigma}$ as 0 .) Before defining $\delta: C^{n}(\mathbf{A}, \mathbf{M}) \rightarrow C^{n+1}(\mathbf{A}, \mathbf{M})$, we establish some notation: $T(i, j)=T^{i j}$ when $i \leqslant j$, and is 0 otherwise; $T_{v}(\sigma, \tau)=T(v \sigma, v \tau)$; $T_{u}(\sigma, \tau)=T(u \sigma, u \tau)$; and $T^{\sigma}=T(v \sigma, u \sigma)$. When $\sigma \in \Sigma_{p}$ and $\Gamma \in C^{n}(\mathbf{A}, \mathbf{M})(p \leqslant$ $n+1)$, define $\Gamma^{\partial \sigma}$ by

$$
\Gamma^{\partial \sigma}=\sum_{0 \leqslant r \leqslant p}(-1)^{p-r} T_{v}\left(\sigma, \sigma_{r}\right) \Gamma^{\sigma_{r}} \varphi_{u}\left(\sigma_{r}, \sigma\right) \in C^{n-p+1}\left(A^{u \sigma}, M^{v \sigma}\right) .
$$

[N.B.: $r \neq 0 \Rightarrow T_{v}\left(\sigma, \sigma_{r}\right)=\mathrm{id}$ and $r \neq p \Rightarrow \varphi_{u}\left(\sigma_{r}, \sigma\right)=\mathrm{id}$; so it is "safe" to think of $\Gamma^{\partial \sigma}$ as $\Sigma(-1)^{p-r} \Gamma^{\sigma_{r}}$.] The coboundary in $C^{*}(\mathbf{A}, \mathbf{M})$ is given by

$$
(\delta \Gamma)^{\sigma}=\Gamma^{\partial \sigma}+(-1)^{\sigma} \delta \Gamma^{\sigma} .
$$

The first summand vanishes when $\sigma=(i) \in \Sigma_{0}$; the second when $\sigma \in \Sigma_{n+1}$. Otherwise, a typical component of $\delta \Gamma$ follows the models

$$
(\delta \Gamma)^{i j}=T^{i j} \Gamma^{j}-\Gamma^{i} \varphi^{i j}-\delta \Gamma^{i j}
$$

and

$$
(\delta \Gamma)^{i j k}=T^{i j} \Gamma^{j k}-\Gamma^{i k}+\Gamma^{i j} \varphi^{j k}+\delta \Gamma^{i j k} .
$$

An easy computation confirms that $\delta^{2}=0$. Of course, when $\mathbf{A}=\varphi: B \rightarrow A$ and $\mathbf{M}=T: N \rightarrow M$, the complex introduced in $\S 3$ and $C^{*}(\mathbf{A}, \mathbf{M})$ are the same.

Recall that a Hochschild $n$-cochain $f$ is normal if $f\left(x_{1}, \ldots, x_{n}\right)=0$ whenever any $x_{i}$ is 1. The normal cochains form a subcomplex and the inclusion of complexes induces an isomorphism of the "normal cohomology" with the "full cohomology." This follows trivially from: If $\delta f$ is normal then there exists an $(n-1)$-cochain $\hat{f}$ such that $f-\delta \hat{f}$ is normal. We recall the proof here since the technique will be needed for an important lemma in $\S 17$ : Assume, inductively, that $f$ is normal through place $r-1$, i.e., $f\left(x_{1}, \ldots, x_{n}\right)=0$ whenever $x_{i}=1$, some $i<r$. Define an $(n-1)$-cochain $h$ by

$$
h\left(x_{1}, \ldots, x_{n-1}\right)=f\left(x_{1}, \ldots, x_{r-1}, 1, x_{r}, \ldots, x_{n-1}\right) .
$$

Then $h$ is normal through place $r-1$ and, hence, so is $f-\delta h$. But

$$
(f-\delta h)\left(x_{1}, \ldots, x_{r-1}, 1, x_{r}, \ldots, x_{n}\right)=(-1)^{r+1} \delta f\left(x_{1}, \ldots, x_{r-1}, 1,1, x_{r}, \ldots, x_{n}\right)
$$

and the latter is 0 since $\delta f$ is normal. Hence $f-\delta h$ is normal through place $r$ and the induction proceeds.

We shall call a diagram cochain $\Gamma$ normal if each $\Gamma^{\sigma}$ is normal. Once again the normal cochains form a subcomplex and the inclusion induces an isomorphism of cohomologies: suppose, as before, that $\delta \Gamma$ is normal. Assume, inductively, that $\Gamma^{\sigma}$ is normal for every $\sigma \in \Sigma_{r}, r<p$. If $\sigma \in \Sigma_{p}$ then $\delta \Gamma^{\sigma}=(-1)^{\sigma}\left[(\delta \Gamma)^{\sigma}-\Gamma^{\partial \sigma}\right]$, which is normal. Hence $\Gamma^{\sigma}-\delta \hat{\Gamma}^{\sigma}$ is normal for some $\hat{\Gamma}^{\sigma}$. Define $\Delta$ by $\Delta^{\sigma}=(-1)^{\sigma} \hat{\Gamma}^{\sigma}$ if $\sigma \in \Sigma_{p}$, and $\Delta^{\sigma}=0$ otherwise. Clearly, $(\Gamma-\delta \Delta)^{\sigma}$ is normal for every $\sigma \in \Sigma_{r}$, $r<p+1$, and the induction proceeds. 
It is important to recognize that $C^{*}(\mathbf{A}, \mathbf{M})$ is the total complex of a double complex: start by setting $C^{q}=\prod_{i \leqslant j} C^{q}\left(\mathbf{A}^{j}, \mathbf{M}^{i}\right)$. Then define $C^{q . p}$ and two anticommuting coboundaries $\delta_{\mathrm{I}}: C^{q, p} \rightarrow C^{q+1 . p}$ and $\delta_{\mathrm{II}}: C^{q, p} \rightarrow C^{q, p+1}$ as follows:

$$
\begin{aligned}
& C^{q . p}=\left\{\Gamma: \Sigma_{p} \rightarrow C^{q} \mid \Gamma^{\sigma} \in C^{q}\left(\mathbf{A}^{u \sigma}, \mathbf{M}^{v \sigma}\right)\right\} ; \\
& \left(\delta_{\mathrm{I}} \Gamma\right)^{\sigma}=(-1)^{\sigma} \delta \Gamma^{\sigma} ; \text { and }\left(\delta_{\mathrm{II}} \Gamma\right)^{\sigma}=\Gamma^{\partial \sigma} .
\end{aligned}
$$

(Note that this is not a subcomplex of $\operatorname{Hom}_{g p s}\left(C_{p}, C^{q}\right)$ since $\delta_{\mathrm{II}}$ is not the coboundary induced by $\partial: C_{p} \rightarrow C_{p-1}$.) It is clear that $C^{n}(\mathbf{A}, \mathbf{M})=\Pi_{q+p=n} C^{q . p}$ and $\delta=\delta_{\mathrm{I}}+\delta_{\mathrm{II}}$. Let $\mathbf{K}$ be the diagram determined by $\mathbf{K}^{i}=k$. Then this description of $C^{*}(\mathbf{K}, \mathbf{K})$ shows that $H^{*}(\mathbf{K}, \mathbf{K})$ is precisely the simplicial cohomology $H^{*}(\Sigma, k)-\mathrm{a}$ theme we shall discuss in a forthcoming paper.

Let $\mathrm{Cyl}{ }^{*}(\mathbf{A}, \mathbf{M})$ denote the mapping cylinder of $\prod_{i} C^{*}\left(\mathbf{A}^{i}, \mathbf{M}^{i}\right) \rightarrow \Pi_{i<j} C^{*}\left(\mathbf{A}^{j}, \mathbf{M}^{i}\right)$, $\left\{\Gamma^{i}\right\} \rightarrow\left\{T^{i j} \Gamma^{j}-\Gamma^{i} \varphi^{i j}\right\}$; its cohomology will be called $\mathrm{Hyl}^{*}(\mathbf{A}, \mathbf{M})$. Truncation gives a cochain map $C^{*}(\mathbf{A}, \mathbf{M}) \rightarrow \mathrm{Cyl}^{*}(\mathbf{A}, \mathbf{M})$ which is an isomorphism whenever $\Sigma_{p}=\varnothing$ for all $p \geqslant 2$. The definitions of $\bar{\circ},[-,-]$, and $\smile$ in $\$ \S 4-5$ carry over in an obvious way to $\mathrm{Cyl}^{*}(\mathbf{A}, \mathbf{A})$. Moreover, an easy extension of the arguments in $[\mathbf{S c h}]$ shows that these induce Lie and cup products on $\mathrm{Hyl}^{*}(\mathbf{A}, \mathbf{A})$. At the same time, if the CCT applies to $\mathbf{A}$, there are Lie and cup products on $H^{*}(\mathbf{A}, \mathbf{A})$. We conjecture that these products always exist and that $H^{*}(\mathbf{A}, \mathbf{A}) \rightarrow \mathrm{Hyl}^{*}(\mathbf{A}, \mathbf{A})$ is a morphism for each product. Added in proof: Explicit descriptions for $\bar{\circ}$ and $\smile$ on $C^{*}(\mathbf{A}, \mathbf{A})$ will appear in Simplicial cohomology is Hochschild cohomology, J. Pure Appl. Algebra.

Any bimodule morphism $\mathbf{M} \rightarrow \mathbf{N}$ (allowable or not) induces a cochain map $C^{*}(\mathbf{A}, \mathbf{M}) \rightarrow C^{*}(\mathbf{A}, \mathbf{N})$ and, so, the diagram-cohomology is a functor $H^{*}(\mathbf{A},-)$. More is true: an allowable short exact sequence of A-bimodules, $E: 0 \rightarrow \mathbf{M}_{1} \rightarrow \mathbf{M}_{2} \rightarrow \mathbf{M}_{3}$ $\rightarrow 0$, induces the usual long exact cohomology sequence

$$
\cdots \rightarrow H^{n}\left(\mathbf{A}, \mathbf{M}_{1}\right) \rightarrow H^{n}\left(\mathbf{A}, \mathbf{M}_{2}\right) \rightarrow H^{n}\left(\mathbf{A}, \mathbf{M}_{3}\right) \stackrel{\delta}{\rightarrow} H^{n+1}\left(\mathbf{A}, \mathbf{M}_{1}\right) \rightarrow \cdots .
$$

That is, we have

THEOREM. $H^{*}(\mathbf{A},-)$ is a relative $\delta$-functor.

Proof. The long exact sequence will be a consequence of the snake lemma once we show $C^{n}(\mathbf{A}, E)$ to be exact for all $n$. For this, simply note that for any $\sigma \in \Sigma$, $E^{v \sigma}: 0 \rightarrow \mathbf{M}_{1}^{v \sigma} \rightarrow \mathbf{M}_{2}^{v \sigma} \rightarrow \mathbf{M}_{3}^{v \sigma} \rightarrow 0$ is an allowable short exact sequence and, so, $C^{q}\left(\mathbf{A}^{u \sigma}, E^{v \sigma}\right)$ is exact for all $q$. But then

$$
C^{n}(\mathbf{A}, E)=\prod_{p \leqslant n} \prod_{\sigma \in \Sigma_{p}} C^{n-p}\left(\mathbf{A}^{u \sigma}, \mathbf{M}^{v \sigma}\right)
$$

is exact, as required.

A subcomplex of $C^{*}(\mathbf{A}, \mathbf{M})$ plays an important role in the next section-it classifies infinitesimal deformations. An $n$-cochain $\Gamma$ is simple if $\Gamma^{\sigma}=0$ for every $\sigma \in \Sigma_{n}$. The subcomplex of simple cochains is denoted by $C_{s}^{*}(\mathbf{A}, \mathbf{M})$; the notations $Z_{s}^{*}(\mathbf{A}, \mathbf{M}), B_{s}^{*}(\mathbf{A}, \mathbf{M})$, and $H_{s}^{*}(\mathbf{A}, \mathbf{M})$ have the expected interpretations. Of course, $H_{s}^{*}(\mathbf{A},-)$ is a relative $\delta$-functor, and the inclusion $C_{s}^{*}(\mathbf{A},-) \rightarrow C^{*}(\mathbf{A},-)$ induces a 
map of $\delta$-functors, $\zeta_{-}^{*}: H_{s}^{*}(\mathbf{A},-) \rightarrow H^{*}(\mathbf{A},-)$, whose kernel is given by

$$
\operatorname{ker} \zeta_{\mathbf{M}}^{*}=\left(B^{*}(\mathbf{A}, \mathbf{M}) \cap Z_{s}^{*}(\mathbf{A}, \mathbf{M})\right) / B_{s}^{*}(\mathbf{A}, \mathbf{M}) .
$$

This map is an isomorphism in every dimension beyond $1+\operatorname{dim} \Sigma$; it is an epimorphism in dimension equal to $\operatorname{dim} \Sigma$. In the commutative case it is also a monomorphism in every dimension: suppose $\Gamma$ is an $(n-1)$-cochain. Observe that if $\sigma \in \Sigma_{n-1}$ then $\Gamma^{\sigma}$ is just an element of $\mathbf{M}^{v \sigma}$, which is a symmetric $\mathbf{A}^{u \sigma}$-bimodule; so $\delta \Gamma^{\sigma}=0$. Define a simple $(n-1)$-cochain $\Gamma_{0}$ by $\Gamma_{0}^{\sigma}=\Gamma^{\sigma}$ for $\sigma \in \Sigma_{p}, p<n-1$. Then $\delta \Gamma=\delta \Gamma_{0}$ if and only if $\delta \Gamma \in Z_{s}^{n}(\mathbf{A}, \mathbf{M})$. In particular, $\operatorname{ker} \zeta_{\mathbf{M}}^{*}=0$.

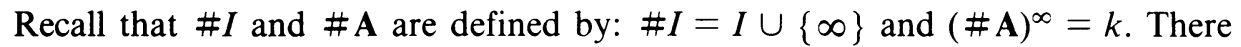
are natural isomorphisms \#: $H_{s}^{*}(\mathbf{A}, \mathbf{A}) \rightarrow H_{s}^{*}(\# \mathbf{A}, \# \mathbf{A})$ and $\#: H^{*}(\mathbf{A}, \mathbf{A}) \rightarrow$ $H^{*}(\# \mathbf{A}, \# \mathbf{A})$ which commute with $\zeta_{\mathrm{A}}^{*}$, namely, send a normal cocycle $\Gamma$ to $\# \Gamma$, where $(\# \Gamma)^{\sigma}=0$ if $u \sigma=\infty$, and $(\# \Gamma)^{\sigma}=\Gamma^{\sigma}$ otherwise.

It will regularly be convenient to use a suggestive representation for an $n$-cochain $\Gamma$. We write $\Gamma=\left\{\Gamma^{i} ; \Gamma^{i j} ; \Gamma^{i j k} ; \ldots\right\}$ where entry $p$ corresponds to a "generic" $(p-1)$-simplex. So a simple two-cochain $\Gamma$ may be written $\Gamma=\left\{\Gamma^{i} ; \Gamma^{i j} ; 0\right\}$.

Recall that $\mathbf{K}$ is the diagram determined by $\mathbf{K}^{i}=k$. There is a natural morphism $\mathbf{K} \stackrel{i}{\rightarrow} \mathbf{A}$ which induces a cochain map $C^{*}(\mathbf{A},-) \stackrel{i^{*}}{\rightarrow} C^{*}(\mathbf{K},-)$. We shall write $C_{N}^{*}(\mathbf{A}, \mathbf{M})$ for the subcomplex of $C^{*}(\mathbf{A}, \mathbf{M})$ consisting of the normal cochains. (N.B.: These complexes have the same cohomology.) If $\Gamma \in C_{N}^{n}(\mathbf{A}, \mathbf{M})$, then $i^{*} \Gamma=\left\{0 ; 0 ; \ldots ; \Gamma^{\sigma}\right\}$, which is normal. Conversely, a normal cochain $\Delta \in C_{N}^{n}(\mathbf{K}, \mathbf{M})$ has the form $\Delta=$ $\left\{0 ; 0 ; \ldots ; \Delta^{\sigma}\right\}$ and, so, may be viewed as an element of $C_{N}^{n}(\mathbf{A}, \mathbf{M})$. Thus there are $k$-module morphisms $C_{N}^{n}(\mathbf{K},-) \rightarrow C_{N}^{n}(\mathbf{A},-)$ which clearly split $i^{*}$. However, these morphisms comprise a cochain map if and only if $\mathbf{A}$ is commutative. This proves the second assertion of the

Lemma. $0 \rightarrow \operatorname{ker} \zeta_{-}^{*} \rightarrow H_{s}^{*}(\mathbf{A},-) \rightarrow H^{*}(\mathbf{A},-) \stackrel{i^{*}}{\rightarrow} H^{*}(\mathbf{K},-)$ is exact. Moreover, in the commutative case we have a split exact sequence

$$
0 \rightarrow H_{s}^{*}(\mathbf{A},-) \rightarrow H^{*}(\mathbf{A},-) \stackrel{i^{*}}{\rightarrow} H^{*}(\mathbf{K},-) \rightarrow 0 .
$$

Proof. We need only establish the exactness of the first sequence at $H^{*}(\mathbf{A},-)$. We may and do assume all cochains to be normal. Suppose $\Gamma \in C_{N}^{n}(\mathbf{A}, \mathbf{M})$ and $i^{*} \Gamma=\delta_{\mathbf{K}} \Delta$ with $\Delta=\left\{0 ; 0 ; \ldots ; \Delta^{\sigma}\right\}$. Then viewing $\Delta$ in $C^{n-1}(\mathbf{A}, \mathbf{M})$ we see that $\Gamma^{\sigma}=\left(\delta_{\mathbf{K}} \Delta\right)^{\sigma}=$ $\left(\delta_{\mathrm{A}} \Delta\right)^{\sigma}$ for $\sigma \in \Sigma_{n}$. So $\Gamma-\delta_{\mathrm{A}} \Delta$ is a simple cochain and we have the required exactness.

In the commutative case we may replace the Hochschild complexes by Harrison complexes in the discussions above. The resulting complexes and their cohomologies are denoted $\mathrm{Ch}^{*}(\mathbf{A}, \mathbf{M}), \mathrm{Ch}_{s}^{*}(\mathbf{A}, \mathbf{M}), \operatorname{Har}^{*}(\mathbf{A}, \mathbf{M})$, and $\operatorname{Har}_{s}^{*}(\mathbf{A}, \mathbf{M})$. As expected, there are morphisms $\operatorname{Har}^{*}(\mathbf{A}, \mathbf{M}) \rightarrow H^{*}(\mathbf{A}, \mathbf{M})$ and $\operatorname{Har}_{s}^{*}(\mathbf{A}, \mathbf{M}) \rightarrow H_{s}^{*}(\mathbf{A}, \mathbf{M})$.

Note that if $M$ is an $A$-bimodule, then $H^{0}(A, M)$ is the "center" of $M$, i.e., $\{m \in M \mid a m=m a$, all $a \in A\}=\operatorname{Hom}_{A}(A, M)$. For diagrams, $H^{0}(\mathbf{A}, \mathbf{M})$ consists of all sets of central elements $\left\{m^{i} \in \mathbf{M}^{i}\right\}$ with $T^{i j} m^{j}=m^{i}$ whenever $i \leqslant j$. This is precisely the same as the set of A-bimodule morphisms $\mathbf{A} \rightarrow \mathbf{M}, \operatorname{Hom}_{\mathbf{A}}(\mathbf{A}, \mathbf{M})$. (Send $a^{i} \in \mathbf{A}^{i}$ to $a^{i} m^{i}$.) Of course, $H_{s}^{0}(\mathbf{A}, \mathbf{M})=0$. 
8. Infinitesimals and diagram extensions. For any $k$-algebra $A$ and $A$-bimodule $M$ there are two classical descriptions of $H^{2}(A, M)$ : (1) it is the module of additively split singular extensions of $A$ by $M$ [Ho]; and (2) (when $M=A$ ) it is the module of infinitesimal deformations of $A$ [G2]. We shall prove below that neither of these descriptions generally applies to the diagram cohomology $H^{2}(\mathbf{A}, \mathbf{M})$; however, both extend to $H_{s}^{2}(\mathbf{A}, \mathbf{M})$. Hence it is natural to think of an infinitesimal as a "diagram extension" rather than as a representative of a diagram cohomology class. Given these results it is fair to ask why we prefer $H^{*}(\mathbf{A},-)$ to $H_{s}^{*}(\mathbf{A},-)$. The answer is contained in the next section wherein we prove that $H^{*}(\mathbf{A},-)$ is universal and, hence, coincides with the (relative) Yoneda theory of A-MOD.

We first attend to the infinitesimals. Let $\mathbf{A}_{t}$ be a deformation of $\mathbf{A}$. Let $\alpha_{t}^{i}=\alpha^{i}+$ $\alpha_{1}^{i} t+\alpha_{2}^{i} t^{2}+\cdots$ be the deformation of $\mathbf{A}^{i}$ and write $\varphi_{t}^{i j}=\varphi^{i j}+\varphi_{1}^{i j}+\varphi_{2}^{i j} t^{2}+\cdots$. The $n$ th-cochain of $\mathbf{A}_{t}$ is $\Gamma_{n}=\left\{\alpha_{n}^{i} ; \varphi_{n}^{i j} ; 0\right\} \in C^{2}(\mathbf{A}, \mathbf{A})$. One immediately sees that $\Gamma_{1}$ — the infinitesimal-lies in $Z_{s}^{2}(\mathbf{A}, \mathbf{A}) \cdot\left[\varphi^{i j} \alpha_{1}^{j}-\alpha_{1}^{i} \varphi^{i j}-\delta \varphi_{1}^{i j}=0\right.$ since $\varphi_{t}^{i j}$ is an algebra morphism; $\varphi^{i j} \varphi_{1}^{j k}-\varphi_{1}^{i k}+\varphi_{1}^{i j} \varphi^{j k}=0$ because $\varphi_{t}^{i j} \varphi_{t}^{j k}=\varphi_{t}^{i k}$.] Morecver, every element of $Z_{s}^{2}(\mathbf{A}, \mathbf{A})$ defines a deformation (modulo $t^{2}$ ) and, so, is a possible infinitesimal. Notice, however, that every other element of $Z^{2}(\mathbf{A}, \mathbf{A})$ is a priori ineligible to be an infinitesimal-so it would be unreasonable to construe $Z^{2}(\mathbf{A}, \mathbf{A})$ as the infinitesimals. Still worse, the lemma of $\S 3$ does not generalize to $H^{2}(\mathbf{A}, \mathbf{A})$; however, it does extend to $H_{s}^{2}(\mathbf{A}, \mathbf{A})$. Specifically,

Lemma. There is a deformation equivalent to $\mathbf{A}_{t}$ having infinitesimal $\left\{\Gamma^{i} ; \Gamma^{i j} ; 0\right\}$ if and only if $\left\{\alpha_{1}^{i} ; \varphi_{1}^{i j} ; 0\right\}-\left\{\Gamma^{i} ; \Gamma^{i j} ; 0\right\} \in B_{s}^{2}(\mathbf{A}, \mathbf{A})$. Hence, $H_{s}^{2}(\mathbf{A}, \mathbf{A})$ is the module of infinitesimal deformations.

Proof. For the lemma in $\S 3$ (with $\mathbf{A}=$ a morphism) we did two things: (1) we proved this lemma; and (2) we showed that $B^{2}(\varphi, \varphi)=B_{s}^{2}(\varphi, \varphi)$. The first proof, with some obvious modifications, works for arbitrary $\mathbf{A}$. The second statement is false in general-even if $Z^{2}(\mathbf{A}, \mathbf{A})=Z_{s}^{2}(\mathbf{A}, \mathbf{A})$; that is why we must settle for $H_{s}^{2}(\mathbf{A}, \mathbf{A})$ rather than a submodule of $H^{2}(\mathbf{A}, \mathbf{A})$.

This lemma prevents us from viewing $H^{2}(\mathbf{A}, \mathbf{A})$ as containing the infinitesimal deformations-unless $\zeta_{\mathrm{A}}^{2}: H_{s}^{2}(\mathbf{A}, \mathbf{A}) \rightarrow H^{2}(\mathbf{A}, \mathbf{A})$ is a monomorphism. The deformations whose infinitesimals lie in $\operatorname{ker} \zeta_{\mathbf{A}}^{2}$ are precisely the ones which are inessential to order 1.

LEMMA. If $\mathbf{A}_{t}$ and $\overline{\mathbf{A}}_{t}$ are deformations of $\mathbf{A}$ and $\Gamma_{r}=\bar{\Gamma}_{r}$ for $r<n$, then $\Gamma_{n}-\bar{\Gamma}_{n} \in$ $Z_{s}^{2}(\mathbf{A}, \mathbf{A})$. Moreover, if they are inequivalent there is a deformation $\hat{\mathbf{A}}_{t}$ and an integer $n$ such that: $\hat{\mathbf{A}}_{t}$ is equivalent to $\overline{\mathbf{A}}_{t} ; \hat{\Gamma}_{r}=\Gamma_{r}, r<n ; \hat{\Gamma}_{n}-\Gamma_{n} \notin B_{s}^{2}(\mathbf{A}, \mathbf{A})$.

Proof. The argument that $\Gamma_{1} \in Z_{s}^{2}(\mathbf{A}, \mathbf{A})$ also shows $\Gamma_{n}-\bar{\Gamma}_{n} \in Z_{s}^{2}(\mathbf{A}, \mathbf{A})$. If $\Gamma_{n}-\bar{\Gamma}_{n}=\delta \Delta$, where $\Delta=\left\{\Delta^{i} ; 0\right\}$, define $F_{t, n}^{i}: \mathbf{A}^{i}[[t]] \rightarrow \mathbf{A}^{i}[[t]]$ by $F_{t, n}^{i}\left(a^{i}\right)=a^{i}-$ $\Delta^{i}\left(a^{i}\right) t^{n}$ and $\hat{\alpha}_{t}^{i}$ by $F_{t, n}^{i} \bar{\alpha}_{t}^{i}\left(a_{1}, a_{2}\right)=\hat{\alpha}_{t}^{i}\left(F_{t, n}^{i} a_{1}, F_{t, n}^{i} a_{2}\right)$. Then $F_{t, n}: \overline{\mathbf{A}}_{t} \rightarrow \hat{\mathbf{A}}_{t}$ is an equivalence and $\hat{\Gamma}_{r}=\Gamma_{r}$ for $r<n+1$. Suppose we can continue in this fashion. Then $F_{t}^{i}=\cdots \circ F_{t, n+1}^{i} \circ F_{t, n}^{i}$ is a well-defined power series and gives an equivalence $\overline{\mathbf{A}}_{t} \rightarrow \hat{A}_{t}$. Hence this process must terminate at some $n$ with the required deformation. 
This lemma will be used in the proof of the theorem in $\S 21$, where the case $\mathbf{A}=\mathrm{a}$ finite diagram is of particular importance.

We now turn our attention to diagram extensions. Specifically, (E): $0 \rightarrow \mathbf{M} \rightarrow$ $\mathbf{E} \stackrel{\sigma}{\rightarrow} \mathbf{A} \rightarrow 0$ is a singular extension of $\mathbf{A}$ by $\mathbf{M}$ if: $\mathbf{E}$ is a diagram of $k$-algebras and $\sigma$ is a natural transformation; $\left(\mathbf{M}^{i}\right)^{2}=0$ in $\mathbf{E}^{i}$; and the operations of $\mathbf{E}^{i}$ and $\mathbf{A}^{i}$ on $\mathbf{M}^{i}$ coincide (e.g., $m^{i} e^{i}=m^{i} \boldsymbol{\sigma}^{i}\left(e^{i}\right)$ ) (see [Ho]). It is additively split if there are $k$-module morphisms $s^{i}: \mathbf{A}^{i} \rightarrow \mathbf{E}^{i}$ splitting $\sigma^{i}$. [N.B.: $\left\{s^{i}\right\}$ need not define a K-module morphism $\mathbf{A} \rightarrow \mathbf{E}$.] Two extensions, $(\mathbf{E})$ and $(\tilde{\mathbf{E}})$, are equivalent if there is a natural transformation $t: \mathbf{E} \rightarrow \tilde{\mathbf{E}}$ inducing the identities on $\mathbf{M}$ and $\mathbf{A}$. The set of equivalence classes of additively split singular extensions is a $k$-module in which addition is given by Baer sum $[\mathbf{M}]$. We denote it by exal( $\mathbf{A}, \mathbf{M})$. Using pushouts and pullbacks we see that exal(-,-) is a covariant functor of the second variable and a contravariant functor of the first variable.

LEMMA. exal $(\mathbf{A}, \mathbf{M}) \cong H_{s}^{2}(\mathbf{A}, \mathbf{M})$

Proof. Let (E) represent a class in exal $(\mathbf{A}, \mathbf{M})$, say $\mathbf{E}=\left\{\mathbf{E}^{i}, \psi^{i j}\right\}$. Choose splittings $\left\{s^{i}\right\}$ and define $\Gamma=\Gamma_{\mathbf{E}} \in C_{s}^{2}(\mathbf{A}, \mathbf{M})$ by $\Gamma^{i}\left(a, a^{\prime}\right)=s^{i}(a) s^{i}\left(a^{\prime}\right)-s^{i}\left(a a^{\prime}\right)$ and $\Gamma^{i j}=\psi^{i j} s^{j}-s^{i} \varphi^{i j}$. Showing $\Gamma$ to be a two-cocycle is routine. A different choice of splittings, say $\tilde{s}^{i}$, would determine a different cocycle, say $\tilde{\Gamma}$; however, $\Gamma=\tilde{\Gamma}+$ $\delta\left\{\tilde{s}^{i}-s^{i} ; 0\right\}$. If $t: \mathbf{E} \rightarrow \tilde{\mathbf{E}}$ is an equivalence choose $t^{i} s^{i}$ as the splitting $\mathbf{A}^{i} \rightarrow \tilde{\mathbf{E}}^{i}$. Then $\Gamma_{E}=\Gamma_{\tilde{E}}$ and, so, we have a morphism exal $(\mathbf{A}, \mathbf{M}) \rightarrow H_{s}^{2}(\mathbf{A}, \mathbf{M})$.

Now let $\Gamma=\left\{\Gamma^{i} ; \Gamma^{i j} ; 0\right\}$ be a simple two-cocycle. Define $\mathbf{E}_{\Gamma}=\left\{\mathbf{E}^{i}, \psi^{i j}\right\}$ as follows: as a $k$-module $\mathbf{E}^{i}=\mathbf{A}^{i} \times \mathbf{M}^{i}$; in $\mathbf{E}^{i}$,

$$
\begin{aligned}
(a, m)\left(a^{\prime}, m^{\prime}\right) & =\left(a a^{\prime}, a m^{\prime}+m a^{\prime}+\Gamma^{i}\left(a, a^{\prime}\right)\right) ; \\
\psi^{i j}((a, m)) & =\left(\varphi^{i j}(a), T^{i j}(m)+\Gamma^{i j}(a)\right) .
\end{aligned}
$$

Routine calculations show that $\mathbf{E}_{\Gamma}$ is a diagram of algebras and $\left(\mathbf{E}_{\Gamma}\right)$ is an additively split singular extension. If $\Delta \in C_{s}^{1}(A, M)$ then $\left(\mathbf{E}_{\Gamma}\right)$ is equivalent to $\left(\mathbf{E}_{\Gamma+\delta \Delta}\right)$ : just define $\mathbf{E}_{\Gamma} \stackrel{t}{\rightarrow} \mathbf{E}_{\Gamma+\delta \Delta}$ by $t^{i}((a, m))=\left(a, m-\Delta^{i}(a)\right)$. Hence there is a morphism $H_{s}^{2}(\mathbf{A}, \mathbf{M}) \rightarrow \operatorname{exal}(\mathbf{A}, \mathbf{M})$.

Standard arguments now show the two morphisms we have constructed to be inverse $k$-module homomorphisms.

The kernel of $\zeta_{\mathbf{M}}^{2}: H_{s}^{2}(\mathbf{A}, \mathbf{M}) \rightarrow H^{2}(\mathbf{A}, \mathbf{M})$ consists of the inessential extensions. In the commutative case there are no inessential extensions. Likewise, there are none when $\mathbf{A}$ is an algebra or a morphism-for then $\zeta_{\mathbf{M}}^{2}$ is an isomorphism. For the simplest nontrivial inessential extension let $I$ be the poset $\{0,1,2\}$ with $1>0,2>0$. Let $A$ be a noncommutative algebra-say $a b \neq b a$. Define $\mathbf{A}$ and $\mathbf{M}$ by $\mathbf{A}^{i}=A$, $\varphi^{i j}=\mathrm{id}, \mathbf{M}^{1}=0=\mathbf{M}^{2}$, and $\mathbf{M}^{0}=A$. Finally, define $\mathbf{E}$ by: $\mathbf{E}^{1}=A=\mathbf{E}^{2}, \mathbf{E}^{0}=$ $A \oplus A, \psi^{01}(x)=(x, 0)$ and $\psi^{02}(x)=(x, x b-b x)=(x,(\delta b) x)$. Observe that restricting $(\mathbf{E})$ to any proper subset of $I$ produces a split extension, but (E) itself is nontrivial. 
9. Yoneda cohomology. If $M_{0}$ and $M$ are bimodules over a single $k$-algebra $A$, the set of Yoneda equivalence classes of exact sequences $0 \rightarrow M \rightarrow M_{n} \rightarrow \cdots \rightarrow M_{0} \rightarrow 0$ $(n \geqslant 1)$ in $A$-MOD forms in a natural way a $k$-module usually denoted $\operatorname{Ext}^{n}\left(M_{0}, M\right)$; one sets $\operatorname{Ext}^{0}\left(M_{0}, M\right)=\operatorname{Hom}_{A}\left(M_{0}, M\right)$. We shall, however, reserve the notation $\mathrm{Ext}^{n}$ for the submodule of classes of $|-|$-allowable sequences. Since $A$-MOD has enough $|-|$-relative projectives and injectives, $\operatorname{Ext}^{*}(-,-)$ is a universal $|-|$-relative $\delta$-functor in each argument [M, pp. 391-392]. Now

$$
\operatorname{Ext}^{0}(A,-)=\operatorname{Hom}_{A}(A,-)=H^{0}(A,-) .
$$

So, universality in the second argument implies the existence of a unique extension of id: $\operatorname{Ext}^{0}(A,-) \rightarrow H^{0}(A,-)$ to $\operatorname{Ext}^{*}(A,-) \rightarrow H^{*}(A,-)$. In fact, this is a natural isomorphism [M, X.3]. In particular, then, if $n>0, H^{n}(A,-)$ vanishes on $|-|$-relative injectives.

For any diagram A one can form Yoneda equivalence classes of $U$-allowable exact sequences in $\mathbf{A}-\mathrm{MOD}$. The resulting bifunctor $\operatorname{Ext}_{\mathbf{A}}^{*}(-,-)$ is universal in both arguments, as A-MOD has enough $U$-relative projectives and injectives.

TheOREM. $\operatorname{Ext}_{\mathbf{A}}^{*}(\mathbf{A},-) \cong H^{*}(\mathbf{A},-)$.

Proof. $\operatorname{Ext}_{\mathbf{A}}^{0}(\mathbf{A},-)=\operatorname{Hom}_{\mathbf{A}}(\mathbf{A},-)=H^{0}(\mathbf{A},-)$. So there is a natural transformation $\operatorname{Ext}_{\mathbf{A}}^{*}(\mathbf{A},-) \rightarrow H^{*}(\mathbf{A},-)$ extending the identity. If $H^{*}(\mathbf{A},-)$ is also universal, then this will be the required isomorphism. But $H^{*}(\mathbf{A},-)$ is universal if and only if, for $n>0$, it vanishes on enough $U$-relative injectives. Since $H^{*}\left(\mathbf{A}, \Gamma \mathbf{M}_{i}\right)=\Pi H^{*}\left(\mathbf{A}, \mathbf{M}_{i}\right)$ and each such injective is a product of $j$-primitive ones, it suffices to show $H^{n}(\mathbf{A}, \mathbf{I})=0$ for $n>0$ and $\mathbf{I}$ a primitive relative injective.

If $\mathbf{M}$ is any $\mathbf{A}$-bimodule there are natural morphisms $H^{*}(\mathbf{A}, \mathbf{M}) \rightarrow H^{*}\left(\mathbf{A}^{i}, \mathbf{M}^{i}\right)$ induced by the cochain maps $\Gamma \mapsto \Gamma^{i}$. Suppose that we have chosen an index in $I$ - which we shall denote by 0 , that $M$ is an $A^{0}$-bimodule, and that $\mathbf{M}^{i}=M$ for $i \in I_{0}=\{j \geqslant 0\}, \mathbf{M}^{i}=0$ otherwise. (Here $T^{i j}: \mathbf{M}^{j} \rightarrow \mathbf{M}^{i}$ is the identity if $j \geqslant i \geqslant 0$ and is zero otherwise.) Then $H^{*}(\mathbf{A}, \mathbf{M}) \rightarrow H^{*}\left(\mathbf{A}^{0}, M\right)$ is an isomorphism, as we now prove.

If $\Gamma \in C^{n}(\mathbf{A}, \mathbf{M})$ and $\sigma \notin \Sigma\left(I_{0}\right)$ then $\Gamma^{\sigma}=0$, since $\mathbf{M}^{v \sigma}=0$; hence, to describe a cochain we need only define $\Gamma^{\sigma}$ for $\sigma \in \Sigma\left(I_{0}\right)$. Observe that $C^{*}\left(\mathbf{A}^{0}, M\right) \rightarrow C^{*}(\mathbf{A}, \mathbf{M})$, $h \rightarrow\left\{h \varphi^{0 i} ; 0 ; 0 ; \ldots\right\}$ is a cochain map which clearly splits $C^{*}(\mathbf{A}, \mathbf{M}) \rightarrow C^{*}\left(\mathbf{A}^{0}, M\right)$. Hence, $H^{*}(\mathbf{A}, \mathbf{M}) \rightarrow H^{*}\left(\mathbf{A}^{0}, M\right)$ is an epimorphism which we wish to show is also a monomorphism. To this end pick $\Gamma \in Z^{n}(\mathbf{A}, \mathbf{M})$ and let $\Delta=\left\{\Gamma^{0 i} ; \Gamma^{0 i j} ; \Gamma^{0 i j k} ; \ldots\right\}$, that is, when $\sigma=\left(i_{p}<\cdots<i_{0}\right)$ set $0 \sigma=\left(0<i_{p}<\cdots<i_{0}\right)$ and $\Delta^{\sigma}=\Gamma^{0 \sigma}$. (If $i_{p}=0$ then $0 \sigma$ is degenerate and $\Delta^{\sigma}=\Gamma^{0 \sigma}=0$.) We claim that $\Gamma-\delta \Delta=$ $\left\{\Gamma^{0} \varphi^{0 i} ; \ldots\right\}$. Notice that this implies that $C^{*}(\mathbf{A}, \mathbf{M}) \rightarrow C^{*}\left(\mathbf{A}^{0}, M\right) \rightarrow C^{*}(\mathbf{A}, \mathbf{M})$ induces the identity on $H^{*}(\mathbf{A}, \mathbf{M})$ and, so, $H^{*}(\mathbf{A}, \mathbf{M}) \rightarrow H^{*}\left(\mathbf{A}^{0}, M\right)$ is a monomorphism, as desired.

Now, it is clear that $(\Gamma-\delta \Delta)^{0}=\Gamma^{0}$. When $i \neq 0$ we see $(\delta \Delta)^{i}=\delta \Delta^{i}=\delta \Gamma^{0 i}$, while $(\delta \Gamma)^{0 i}=\Gamma^{i}-\Gamma^{0} \varphi^{0 i}-\delta \Gamma^{0 i}$. But $\Gamma$ is a cocycle; so $(\delta \Gamma)^{0 i}=0$ and we have $(\Gamma-\delta \Delta)^{i}=\Gamma^{0} \varphi^{0 i}$. Finally, if $\sigma \in \Sigma_{0}$ then

$$
(\delta \Delta)^{\sigma}=\Delta^{\partial \sigma}+(-1)^{\sigma} \delta \Delta^{\sigma}=\Gamma^{0 \partial \sigma}+(-1)^{\sigma} \delta \Gamma^{0 \sigma} .
$$


On the other hand, since $\Gamma$ is a cocycle we see that

$$
\begin{aligned}
0 & =(\delta \Gamma)^{0 \sigma}=\Gamma^{\partial(0 \sigma)}+(-1)^{0 \sigma} \delta \Gamma^{0 \sigma} \\
& =\Gamma^{\sigma}-\Gamma^{0 \partial \sigma}-(-1)^{\sigma} \delta \Gamma^{0 \sigma}=\Gamma^{\sigma}-(\delta \Delta)^{\sigma},
\end{aligned}
$$

as required.

Of course, the isomorphism establishes the universality of $H^{*}(\mathbf{A},-)$. For if $\mathbf{M}$ is a 0 -primitive relative injective, then $M$ is an $\mathbf{A}^{0}$-relative injective and $H^{n}\left(\mathbf{A}^{0}, M\right)=0$, $n>0$.

Observe that $\mathbf{K}$ is a $\mathbf{K}$-relative projective if and only if $I$ has a maximal element and that, in this case, $H^{*}(\mathbf{K},-)=\operatorname{Ext}_{\mathbf{K}}^{*}(\mathbf{K},-)=0$ (by universality). The lemma of $\S 7$ then shows $H_{s}^{*}(\mathbf{A},-) \rightarrow H^{*}(\mathbf{A},-)$ to be an epimorphism-an isomorphism in the commutative case. Hence when $I$ has a largest element, $H^{2}(\mathbf{A}, \mathbf{A})$ is the module of "essential" infinitesimal deformations.

For a commutative algebra $A, \operatorname{Har}^{*}(A,-)$ is given neither by extensions nor by right-derived functors of $\operatorname{Hom}_{A}(A,-)$, so we should not anticipate an analog of this theorem for $\operatorname{Har}^{*}(\mathbf{A},-)$.

10. The functor !. Let us consider the diagram $\varphi: B \rightarrow A$ and examine the category of left $\varphi$-modules. It is clearly bicomplete and abelian. We denote a sample module by $T: N \rightarrow M$ and use $\langle f, g\rangle: T \rightarrow T^{\prime}$ to mean $f: N \rightarrow N^{\prime}, g: M \rightarrow M^{\prime}$ and $f T^{\prime}=T g$.

$N$.B.: Until the next theorem only, for simplicity, we shall compose morphisms in the diagrammatic order, i.e. $(f g) x=g(f(x))$.

Whenever convenient, we shall use matrix notation for morphisms. Thus, $\left(f_{i j}\right): \bigoplus_{i=1}^{n} X_{i} \rightarrow \bigoplus_{j=1}^{m} Y_{j}$ is described by $\left(f_{i j}\right)\left(x_{1}, \ldots, x_{n}\right)=\left(x_{1}, \ldots, x_{n}\right)\left(f_{i j}\right)$. (Interpret $x_{i} f_{i j}$ as $f_{i j}\left(x_{i}\right)$.) A column matrix may be denoted by a row matrix with a superscript " $t$ ".

It is clear that $(\varphi 0): B \rightarrow A \oplus A$ is a projective object. In fact, it is a small projective. That is, any morphism $\left\langle f,\left(\begin{array}{ll}g_{1} & g_{2}\end{array}\right)^{t}\right\rangle:\left(\begin{array}{ll}\varphi & 0\end{array}\right) \rightarrow \amalg_{\Lambda} T_{\lambda}$ factors through $\amalg_{\Lambda^{\prime}} T_{\lambda}$, where $\Lambda^{\prime} \subseteq \Lambda$ is finite. (Simply let $\Lambda^{\prime}=\left\{\lambda, \lambda^{\prime}\right\}$ where $f(1) \in N_{\lambda}$ and $\left.g_{2}(1) \in M_{\lambda^{\prime}}\right)$ Moreover, $(\varphi 0)$ is a generator. This means that if $\beta=\left\langle\beta_{1}, \beta_{2}\right\rangle$ : $T \rightarrow T^{\prime} \neq 0$, then there is a morphism $\alpha=\left\langle f_{1},\left(g_{1} g_{2}\right)^{t}\right\rangle:(\varphi 0) \rightarrow T$ such that $\alpha \beta \neq 0$. (Observe that $\operatorname{Hom}_{\varphi}((\varphi 0), T) \cong N \oplus M$ by $\alpha \mapsto\left(f(1), g_{2}(1)\right)$. If $\alpha \beta=0$ for all $\alpha$ then $N \subseteq \operatorname{ker} \beta_{1}$ and $M \subseteq \operatorname{ker} \beta_{2}$. So $\beta=0$.)

Any right-complete abelian category with a small projective generator $P$ is equivalent to a category of left modules - the ring being $\operatorname{End}(P)[\mathbf{F r}$, p. 106]. Hence, there is an exact, full embedding of left $\varphi$-modules in the category of left $\operatorname{End}((\varphi 0))$-modules. It is given by $T \mapsto \operatorname{Hom}_{\varphi}((\varphi 0), T)$.

The ring $\operatorname{End}((\varphi 0))$ has a tidy description. Since $B=\operatorname{End}_{B} B$ and $A=\operatorname{End}_{A} A$, an endomorphism of $(\varphi 0)$ has the form $\left\langle b,\left(a_{i j}\right)\right\rangle$, where $b \in B,\left(a_{i j}\right) \in M_{2}(A)$, and

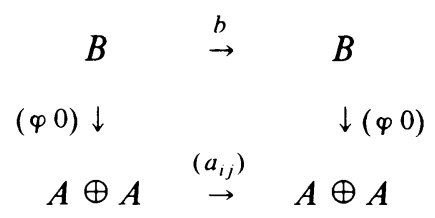


commutes. Tracing $1_{B} \in B$ through the diagram gives $(\varphi(b) 0)=\left(a_{11} a_{12}\right)$. So, as a $k$-module, $\operatorname{End}((\varphi 0))=B \oplus A \oplus A$, under the correspondence $\left\langle b,\left(a_{i j}\right)\right\rangle \mapsto$ $\left(b, a_{22}, a_{21}\right)$. The multiplication in $\operatorname{End}((\varphi 0))$ is just composition. Now, $\left\langle b,\left(a_{i j}\right)\right\rangle\left\langle b^{\prime},\left(a_{i j}^{\prime}\right)\right\rangle=\left\langle b b^{\prime},\left(c_{i j}\right)\right\rangle$, where $c_{11}=\varphi\left(b b^{\prime}\right), c_{12}=0, c_{22}=a_{22} a_{22}^{\prime}$, and $c_{21}=a_{21} \varphi\left(b^{\prime}\right)+a_{22} a_{21}^{\prime}$. In particular, $(0,0,1) \cdot(b, 0,0)=(0,0, \varphi(b))$. Since $(0,0,1)$ "behaves" like $\varphi$, we denote it by $\varphi$. Furthermore, since $(0, a, 0) \varphi=(0, a, 0)(0,0,1)$ $=(0,0, a)$, we write $(0,0, a)=a \varphi$ and represent $\operatorname{End}((\varphi 0))$ as $B \oplus A \oplus A \varphi$. The multiplication is determined by associativity, the products in $B$ and $A$, and the conditions

$$
b a=a b=\varphi a=b \varphi=\varphi^{2}=0 \text { and } \varphi b=\varphi(b) \varphi .
$$

We shall denote this ring by $\varphi$ ! and refer to it as the mapping ring. The unit is $1_{B}+1_{A}$, corresponding to $\langle 1, \mathrm{id}\rangle$.

A similar calculation shows that the left $\varphi$ !-action on $\operatorname{Hom}_{\varphi}((\varphi 0), T)=N \oplus M$ satisfies $b m=a n=\varphi m=0$ and $\varphi n=T(n)$. The embedding is, in fact, an isomorphism: If $V$ is a left $\varphi$ !-module, define $N=1_{B} V, M=1{ }_{A} V$, and $T=$ left multiplication by $\varphi$.

There is one serious drawback to this isomorphism of categories: the image of the left $\varphi$-module $\varphi$ is not the $\varphi$ !-module $\varphi$ !; it is $B \oplus A$. We can remedy this problem by taking a cue from $\varphi$ ! itself. For a left $\varphi$-module $T$ define $T$ ! by $T !=N \oplus M \oplus M \varphi$, with $\varphi n=T(n) \varphi$ and all other products as expected. Now suppose that $T$ is in the full subcategory $\varphi$-MOD (bimodules!). Then, using the right $B$ and $A$ actions, one can parallel (1) and give $T$ ! a right $\varphi$ !-structure. Hence, we have a functor !: $\varphi$-MOD $\rightarrow \varphi$ !-MOD.

We note, in passing, that $\varphi$-MOD is isomorphic to the category of left $\varphi^{e}$-modules, where $\varphi^{e}: B^{e} \rightarrow A^{e}$ is the induced morphism of enveloping algebras. Hence, $\varphi$-MOD $\cong$ left $\varphi^{e}$ !-modules. This suggests the following (open) question: Is there a functor $R$ from morphisms to algebras such that $\varphi$-MOD $\cong R(\varphi)$-MOD? We conjecture that the answer is negative.

If $\mathbf{A}$ is an arbitrary diagram, let $\mathbf{G}$ be defined by $\mathbf{G}^{i}=\amalg_{j \geqslant i} \mathbf{A}^{j}$. Then $\mathbf{G}$ is a projective generator in the category of left $\mathbf{A}$-modules. It is small if and only if $\mathbf{A}$ is finite. The embedding $\operatorname{Hom}_{\mathbf{A}}(\mathbf{G},-)$ is an isomorphism if and only if $\mathbf{G}$ is small. We define the diagram ring of $\mathbf{A}$ to be $\mathbf{A} !=\operatorname{End}(\mathbf{G})$. Proceeding as before, one finds the following description for $\mathbf{A}$ !: As a $k$-module, $\mathbf{A} !=\prod_{i \in I} \amalg_{j \geqslant i} \mathbf{A}^{i} \varphi^{i j}$; multiplication is defined by $\left(a^{i} \varphi^{i j}\right)\left(a^{k} \varphi^{k l}\right)=0$ if $j \neq k$ and

$$
\left(a^{i} \varphi^{i j}\right)\left(a^{j} \varphi^{j k}\right)=a^{i} \varphi^{i j}\left(a^{j}\right) \varphi^{i k}
$$

This can be extended linearly to all of $\mathbf{A}$ !. Let $e^{i}$ be the unit of $\mathbf{A}^{i}$. The unit of $\mathbf{A}$ ! corresponds to id $\in \operatorname{End}(\mathbf{G})$ and is the tuple $\left\langle e^{i} \varphi^{i i}\right\rangle$. Using (infinite) sum notation we denote this by $\Sigma e^{i} \varphi^{i i}$. Observe that $\left\{e^{i} \varphi^{i i}\right\}$ is a set of orthogonal idempotents. Also, $\mathbf{A}^{i} \varphi^{i i}$ is a subalgebra isomorphic to $\mathbf{A}^{i}$. We, therefore, identify these two algebras and abbreviate $a \varphi^{i i}$ to $a$. (So the unit of $\mathbf{A}$ ! is $\sum e^{i}$.) Likewise, we abbreviate $e^{i} \varphi^{i j}$ to $\varphi^{i j}$. These morphisms are thus formally elements of $\mathbf{A}$ ! and we have $\varphi^{i j} \varphi^{j k}=\varphi^{i k}$ (as usual), but $\varphi^{i j} \varphi^{k l}=0$ for $j \neq k$. One must distinguish carefully between $\varphi^{i j}(a)$ and $\varphi^{i j} a$ for $a \in \mathbf{A}^{j}$. (The latter equals $\varphi^{i j}(a) \varphi^{i j}$.) Consequently, we sometimes write $a^{\varphi}$ for $\varphi(a)$ and have $\varphi a=a^{\varphi} \varphi$. 
As before, $\operatorname{Hom}_{\mathbf{A}}(\mathbf{G}, \mathbf{M})=\prod_{I} \mathbf{M}^{i}$ and we wish to modify the embedding. Define !: A-MOD $\rightarrow \mathbf{A}$ !-MOD as follows: As an abelian group, $\mathbf{M} !=\prod_{i \in I} \amalg_{j \geqslant i} \mathbf{M}^{i} \varphi^{i j}$. The operation of $\mathbf{A}$ ! is defined by

$$
\begin{gathered}
\left(a^{i} \varphi^{i j}\right)\left(m^{j} \varphi^{j k}\right)=a^{i} T^{i j}\left(m^{j}\right) \varphi^{i k}, \quad\left(m^{i} \varphi^{i j}\right)\left(a^{j} \varphi^{j k}\right)=m^{i} \varphi^{i j}\left(a^{j}\right) \varphi^{i k}, \\
\left(a^{i} \varphi^{i j}\right)\left(m^{k} \varphi^{k l}\right)=0=\left(m^{i} \varphi^{i j}\right)\left(a^{k} \varphi^{k l}\right) \quad \text { for } j \neq k .
\end{gathered}
$$

We identify $\mathbf{M}^{i}$ with $\mathbf{M}^{i} \varphi^{i i}$ and abbreviate $m^{i} \varphi^{i i}$ to $m^{i}$. Note that $\mathbf{M}$ ! is unital. If $g=\left\langle g^{i}\right\rangle \in \operatorname{Hom}_{\mathbf{A}}(\mathbf{M}, \mathbf{N})$, define $g ! \in \operatorname{Hom}_{\mathbf{A} !}(\mathbf{M} !, \mathbf{N} !)$ by $g !\left(m^{i} \varphi^{i j}\right)=g^{i}\left(m^{i}\right) \varphi^{i j}$.

Note that $\mathbf{M}^{i} \varphi^{i j}$ is a left- $\mathbf{A}^{i}$, right- $\mathbf{A}^{j}$ bimodule. The right module structure is the same as that induced on $\mathbf{M}^{i}$ by $\varphi^{i j}: \mathbf{A}^{j} \rightarrow \mathbf{A}^{i}$.

Let $\mathbf{A}^{\prime}: I \rightarrow$ ALG and $\mathbf{A}: J \rightarrow$ ALG be diagrams. A morphism $\mathbf{A}^{\prime} \rightarrow \mathbf{A}$ will consist of an order-preserving function $p: I \rightarrow J$ (i.e., a functor) and algebra morphisms $f^{i}$ : $\mathbf{A}^{\prime i} \rightarrow \mathbf{A}^{p(i)}$ such that $f^{j} \varphi^{p(i) p(j)}=\varphi^{i j} f^{i}$ whenever $i \leqslant j$. In this way diagrams form a category DIAG. Clearly, !: DIAG $\rightarrow$ ALG is a functor-indeed, an embedding. We also have

THEOREM. !: A-MOD $\rightarrow \mathbf{A}$ !-MOD is a full exact embedding.

Proof. It is clear that ! is an exact embedding. To see that it is full, first observe that multiplication on the right by $\varphi^{i j}$ is an additive isomorphism from $\mathbf{M}^{i}$ to $\mathbf{M}^{i} \varphi^{i j}$. Suppose that $\mathbf{M}, \mathbf{N} \in \mathbf{A}-\mathbf{M O D}$ and $g \in \operatorname{Hom}_{\mathbf{A} !}(\mathbf{M} !, \mathbf{N} !)$. Since $g\left(m^{i} \varphi^{i j}\right)=g\left(m^{i}\right) \varphi^{i j}$ for $m^{i} \in \mathbf{M}^{i}$, one sees that $g$ is determined by its restrictions to the $\mathbf{M}^{i}$. Next, if $m^{i} \in \mathbf{M}^{i}$, then $g\left(m^{i}\right)=g\left(e^{i} m^{i} e^{i}\right)=e^{i} g\left(m^{i}\right) e^{i}$. But $e^{i} \mathbf{N} ! e^{i}=\mathbf{N}^{i}$; so, $g\left(\mathbf{M}^{i}\right) \subseteq \mathbf{N}^{i}$. Define $g^{i}: \mathbf{M}^{i} \rightarrow \mathbf{N}^{i}$ to be $\left.g\right|_{\mathbf{M}^{i}}$. These will unite to define an element of $\operatorname{Hom}_{\mathbf{A}}(\mathbf{M}, \mathbf{N})$ if, for all $j \geqslant i, g^{i} T_{\mathbf{M}}^{i j}=T_{\mathbf{N}}^{i j} g^{j}$. (We use $T_{\mathbf{M}}^{i j}$ and $T_{\mathbf{N}}^{i j}$ to denote the internal morphisms of $\mathbf{M}$ and $\mathbf{N}$, respectively.) Now let $m$ be an element of $\mathbf{M}^{j}$. Then

$$
\varphi^{i j} \cdot g(m)=g\left(\varphi^{i j} m\right)=g\left(T_{\mathbf{M}}^{i j}(m)\right) \varphi^{i j}=g^{i} T_{\mathbf{M}}^{i j}(m) \varphi^{i j} .
$$

But we also have

$$
\varphi^{i j} \cdot g(m)=\varphi^{i j} \cdot g^{j}(m)=T_{N}^{i j} g^{j}(m) \varphi^{i j} .
$$

Since right multiplication by $\varphi^{i j}$ is an additive isomorphism between $\mathbf{M}^{i}$ and $\mathbf{M}^{i} \varphi^{i j}$, it follows that $g^{i} T_{\mathbf{M}}^{i j}(m)=T_{\mathrm{N}}^{i j} g^{j}(m)$, as required.

Since $!$ is a full embedding, there is a natural isomorphism $\omega^{0}: \operatorname{Hom}_{\mathbf{A}}(-,-) \rightarrow$ $\operatorname{Hom}_{A !}(-!,-!)$.

(An observation of $\mathrm{C}$. A. Weibel: $\mathbf{A}$ ! is the incidence matrix ring of a small additive category $\mathbf{C A}$. To obtain $\mathbf{C A}$, first note that every algebra is the endomorphism ring of the single object of a unique additive one object category. So A may be construed as a functor from $I$ to the category of small categories and the Grothendieck construction [Rm, Example 1.1a] may be applied. The resulting category may then be enriched in an obvious way to give $\mathbf{C A}$.)

11. Statement of the Cohomology Comparison Theorem (CCT). The exactness of ! makes it clear that $\operatorname{Ext}_{\mathbf{A} !}^{*}(-!,-!)$ is a $U$-relative $\delta$-functor on A-MOD. Hence, the universality of $\operatorname{Ext}_{\mathbf{A}}^{*}(-,-)$ guarantees that $\omega^{0}$ has a unique extension $\omega^{*}$ : $\operatorname{Ext}_{\mathbf{A}}^{*}(-,-)$ $\rightarrow \operatorname{Ext}_{\mathbf{A} !}^{*}(-!,-!)$. 
Now every allowable exact sequence $E: 0 \rightarrow \mathbf{M} \rightarrow \mathbf{M}_{n} \rightarrow \cdots \rightarrow \mathbf{M}_{1} \rightarrow \mathbf{N} \rightarrow 0$ $(n>0)$ of A-bimodules gives rise to an allowable exact sequence $E$ ! in $\mathbf{A}$ !-MOD. The correspondence $E \mapsto E$ ! clearly preserves equivalence and splicing and is a natural transformation with extends $\omega^{0}$. So it must be $\omega^{*}$. A priori, $\omega^{*}$ need not be an isomorphism - there may be exact sequences connecting $\mathbf{M}$ ! and $\mathbf{N}$ ! in $\mathbf{A}$ !-MOD which do not have the form $E$ !. Nevertheless, we will see that if $\mathbf{A}$ is finite then $\omega^{n}$ is an isomorphism for all $n$. This is the hardest case of the CCT. To state the others we need some definitions.

If we have an algebra morphism $\varphi: B \rightarrow A$ and right $A$-modules $M$ and $N$, then we may consider these as $B$-modules, and we have an inclusion $\operatorname{Hom}_{A}(M, N) \rightarrow$ $\operatorname{Hom}_{B}(M, N)$. This is generally not onto. When it is, we call $\varphi$ a right Hom preserving (H.p.) morphism. Onto morphisms and the canonical morphism of a commutative ring into a localization with respect to some multiplicatively closed set are H.p. If $\varphi$ is both right H.p. and makes $A$ into a flat left $B$-module then we call $\varphi$ a flat H.p. (f.H.p.) morphism; the sidedness will be understood. Flatness is, of course, automatic in the case of localization.

Cohomology Comparison Theorem (CCT). $\omega^{*}$ is an isomorphisin in each of the following cases:

(i) I is finite.

(ii) $I_{p}$ is finite for all $p \in I$; there is a unique maximal element $\infty$ in $I$; and $\mathbf{A}^{p}$ is an f.H.p. $\mathbf{A}^{\infty}$-module for each $p$.

(iii) For all $p, I_{p}$ is finite and $k \rightarrow \mathbf{A}^{p}$ is f.H.p. (Recall that $I_{p}=\{i \in I \mid i \geqslant p\}$.)

We shall refer to these as the "finite," "f.H.p.," and " $k$-f.H.p." cases, respectively. In the last, we can extend $\mathbf{A}$ to an f.H.p. diagram $\mathbf{A}_{+}$by setting $\mathbf{A}_{+}^{\infty}=k$. An A-bimodule $\mathbf{M}$ can be extended to an $\mathbf{A}_{+}$-bimodule $\mathbf{M}_{+}$with $\mathbf{M}_{+}^{\infty}=0$.

The CCT would be trivial if ! preserved either projectives or injectives. In fact it preserves neither. For projectives, let $\mathbf{A}$ be $\varphi: B \rightarrow A$ and $\mathbf{M}$ be $\varphi^{e}: B^{e} \rightarrow A^{e}$, a primitive projective. Then $\varphi !=B \oplus A \oplus A \varphi$ and $\mathbf{M} !=B^{e} \oplus A^{e} \oplus A^{e} \varphi$. In $(\varphi !)^{e}$ consider the ideal $\mathfrak{N}=B^{e} \oplus A \varphi \otimes B^{\mathrm{op}} \oplus A^{e} \oplus A \otimes(A \varphi)^{\mathrm{op}}$, which we view as a $\varphi$ !-bimodule. There is an epimorphism $f: \mathfrak{N} \rightarrow \mathbf{M}$ ! sending $a \varphi \otimes b$ to $\left(a \otimes b^{\varphi}\right) \varphi$ and which is otherwise obvious. However, there is no right inverse $g$ to $f$. For the only possible preimage of $1_{B} \otimes 1_{B}$ is itself, considered as an element of $\mathfrak{T}$, and similarly for $1_{A} \otimes 1_{A}$. If $g$ existed then we would have

$$
g\left(\varphi\left(1_{B} \otimes 1_{B}\right)\right)=g\left(\left(1_{A} \otimes 1_{A}\right) \varphi\right)=g\left(1_{A} \otimes 1_{A}\right) \varphi=\left(1_{A} \otimes 1_{A}\right) \varphi,
$$

while $\varphi g\left(1_{B} \otimes 1_{B}\right)=\varphi\left(1_{B} \otimes 1_{B}\right)=1_{A} \varphi \otimes 1_{B}$, which is different. For injectives, choose an (allowable) $A$-bimodule monomorphism $A \otimes A \rightarrow I$ where $I$ is an $A$-injective, let $\bar{I}$ denote $I$ considered as a $B$-bimodule by means of $\varphi$, and let $\mathbf{I}$ be id: $\bar{I} \rightarrow I$. This is a primitive injective, and $\mathbf{I} !=\bar{I} \oplus I \oplus I \varphi$. Now consider another ideal of $(\varphi !)^{e}$, namely the $\varphi$ !-bimodule

$$
\Re=B \otimes A^{\mathrm{op}} \oplus A \varphi \otimes A^{\mathrm{op}} \oplus B \otimes(A \varphi)^{\mathrm{op}} \oplus A \varphi \otimes(A \varphi)^{\mathrm{op}} .
$$

The submodule $A \varphi \otimes A^{\text {op }} \oplus A \varphi \otimes(A \varphi)^{\text {op }}$ has a monomorphism into I! sending $a \varphi \otimes a^{\prime}$ to $a \otimes a^{\prime} \in I$ and $a \varphi \otimes a^{\prime} \varphi$ to $\left(a \otimes a^{\prime}\right) \varphi \in I \varphi$. This cannot, however, be 
extended to $f: \Re \rightarrow \mathbf{I}$ !, since we would then have $f\left(B \otimes A^{\mathrm{op}}\right)=0$, whence

$$
f\left(\varphi\left(B \otimes A^{\mathrm{op}}\right)\right)=f\left(A \varphi \otimes A^{\mathrm{op}}\right)=0 .
$$

Note that though ! does not preserve all projectives (or all injectives), it remains an open question whether it preserves enough projectives (or enough injectives). Either would suffice for the proof of the theorem.

In the f.H.p. case, if $\mathbf{I}$ is a primitive injective, then $\mathbf{I}$ ! - while not generally injective in $\mathbf{A}$ !-MOD - will be an injective left module over a subalgebra $\mathbf{A} \uparrow$ of the enveloping algebra of $\mathbf{A}$ !. This will yield case (ii) of the CCT.

We should comment that ! also induces a $k$-module morphism $\bar{\omega}_{\mathbf{A}, \mathbf{M}}: \operatorname{exal}(\mathbf{A}, \mathbf{M}) \rightarrow$ $\operatorname{exal}\left(\mathbf{A}\right.$ !, $\mathbf{M}$ !). When $\omega_{\mathbf{A}, \mathbf{M}}^{2}$ is an isomorphism the final lemma of $\S 7$ shows: (1) $\operatorname{ker} \bar{\omega}_{\mathbf{A}, \mathbf{M}}=\operatorname{ker} \zeta_{\mathbf{M}}^{2}=$ the module of inessential extensions, and, (2) $\bar{\omega}_{\mathbf{A}, \mathbf{M}}$ is an epimorphism if $H^{2}(\mathbf{K}, \mathbf{M})=0$.

12. The ring $\mathbf{A} \uparrow$. We shall denote the enveloping algebra $\mathbf{A} !^{e}$ of $\mathbf{A}$ ! by $\mathbf{A} ! \otimes \mathbf{A}$ ! rather than $\mathbf{A} ! \otimes \mathbf{A} !^{\text {op }}$. So multiplication in the second tensor factor is reversed-even though we have dropped the "op". Now define $\mathbf{A} \uparrow, \mathbf{A} \downarrow \subseteq \mathbf{A} !^{e}$ by

$$
\mathbf{A} \uparrow=\prod_{i \in I} \coprod_{j \geqslant i} \prod_{k \geqslant j} \coprod_{l \geqslant k} \mathbf{A}^{i} \varphi^{i j} \otimes \mathbf{A}^{k} \varphi^{k l}
$$

and

$$
\mathbf{A} \downarrow=\prod_{i \in I} \coprod_{j \geqslant i} \prod_{k \neq j} \coprod_{l \geqslant k} \mathbf{A}^{i} \varphi^{i j} \otimes \mathbf{A}^{k} \varphi^{k l}
$$

Then $\mathbf{A} \uparrow$ is a subring and $\mathbf{A} \downarrow$ is a two-sided ideal in $\mathbf{A} !^{e}$. There is an exact sequence $0 \rightarrow \mathbf{A} \downarrow \rightarrow \mathbf{A} !^{e} \rightarrow \mathbf{A} \uparrow \rightarrow 0$; so $\mathbf{A} !^{e}$ is a semidirect product of $\mathbf{A} \downarrow$ and $\mathbf{A} \uparrow$. The unit element of $\mathbf{A} \uparrow$ is $e=\sum_{i \leqslant j} e^{i} \otimes e^{j}$. Note that $\mathbf{A} \downarrow e=0$, so $(\mathbf{A} \downarrow)(\mathbf{A} \uparrow)=0$.

If $\mathscr{N}$ is any left $\mathbf{A} !^{e}$-module, then $e^{\mathscr{R}}$ is a left module over $e \mathbf{A} !^{e} e=\mathbf{A} \uparrow$. So there is a functor $\mathbf{A} !-M O D \rightarrow$ left $\mathbf{A} \uparrow-\mathrm{MOD}, \mathfrak{T} \mapsto e^{\mathfrak{T}}$, which is clearly exact, but may fail to be full. As before, the forgetful functor may be used to define a relative cohomology $\operatorname{Ext}_{\mathbf{A} \uparrow}^{*}(-,-)$ on left $\mathbf{A} \uparrow$-MOD. The universality of $\operatorname{Ext}_{\mathbf{A} !}^{*}(-,-)$ guarantees a unique natural transformation $\alpha^{*}: \operatorname{Ext}_{\mathbf{A} !}^{*}(-,-) \rightarrow \operatorname{Ext}_{\mathbf{A} \uparrow}^{*}(e-, e-)$.

If $\mathbf{M}$ is in $\mathbf{A}-\mathbf{M O D}$, then $e \mathbf{M} !=\mathbf{M}$ !. It is easy to see that the composition of functors A-MOD $\rightarrow \mathbf{A}$ !-MOD $\rightarrow$ left $\mathbf{A} \uparrow-$ MOD, $\mathbf{M} \mapsto \mathbf{M}$ ! is an exact, full embedding. The universality of $\operatorname{Ext}_{\mathbf{A}}^{*}(-,-)$ quarantees a unique extension of the isomorphism $\operatorname{Hom}_{\mathbf{A}}(-,-) \stackrel{\sim}{\rightarrow} \operatorname{Hom}_{\mathbf{A} \uparrow}(-!,-!)$ to a natural transformation $\eta^{*}: \operatorname{Ext}_{\mathbf{A}}(-,-) \rightarrow$ $\operatorname{Ext}_{\mathbf{A} \uparrow}^{*}(-!,-!)$. It follows from uniqueness that $\eta^{*}=\alpha^{*} \omega^{*}$.

Now, if $\mathfrak{N}$ is in $\mathbf{A}$ !-MOD, then $e^{\mathscr{N}}$ is still a module over $\mathbf{A} !^{e}$, as $\mathbf{A} \downarrow e=0$. Meanwhile, $\mathbf{A} !^{e} \rightarrow \mathbf{A} \uparrow$ induces an exact functor left $\mathbf{A} \uparrow-$ MOD $\rightarrow \mathbf{A}$ !-MOD. (The two possible structures on $e^{\mathfrak{R}}$ coincide.) Once again, universality guarantees a unique $\beta^{*}: \operatorname{Ext}_{\mathbf{A} \uparrow}^{*}(-,-) \rightarrow \operatorname{Ext}_{\mathbf{A} !}^{*}(-,-)$.

We wish to examine $\alpha^{*} \beta^{*}$ and $\beta^{*} \alpha^{*}$. If $\Re$ and $\mathscr{T}$ are left $\mathbf{A} \uparrow$-modules, then $e^{\mathscr{T}}=\mathfrak{T}$ and $e \mathfrak{T}=\mathfrak{T}$. Thus, $\alpha^{*} \beta^{*}$ is the unique extension of id: $\operatorname{Hom}_{\mathbf{A} \uparrow}(-,-) \rightarrow$ $\operatorname{Hom}_{\mathbf{A} \uparrow}(-,-)$; that is, $\alpha^{*} \beta^{*}=$ id. Now let $\mathscr{E}: 0 \rightarrow \mathfrak{N} \rightarrow \mathfrak{N}_{n} \rightarrow \cdots \rightarrow \mathfrak{N}_{1} \rightarrow \mathfrak{X} \rightarrow 0$ be an exact sequence of $\mathbf{A}$ !-bimodules. Then

$$
e \varepsilon: 0 \rightarrow e^{\Re} \rightarrow e^{\Re_{n}} \rightarrow \cdots \rightarrow e \Re \Re_{1} \rightarrow e \Re \rightarrow 0
$$


is an exact sequence of left $\mathbf{A} \uparrow$-modules (and, so, is exact in $\mathbf{A}$ !-MOD). Now $\mathcal{E} \mapsto e \mathcal{E}$ is a natural transformation which extends $\operatorname{Hom}_{\mathrm{A} !}(-,-) \rightarrow \operatorname{Hom}_{\mathrm{A} !}(e-, e-)$. So does $\beta^{*} \alpha^{*}$. Thus, $\beta^{*} \alpha^{*}$ must be given by $\mathcal{E} \mapsto e \mathcal{E}$. There is always a morphism of extensions $\mathcal{E} \rightarrow e \mathcal{E}$ in $\mathbf{A}$ !-MOD. When $\Re=e^{\Re}$ and $\Re=e \Re$ this is an equivalence of extensions, as it has equality at both ends. For such $\Re$ and $\Re, \beta^{*} \alpha^{*}$ thus becomes the identity. This applies, in particular, when $\Re=\mathbf{M}$ ! and $\Re=\mathbf{N}$ !. Hence, $\alpha^{*}$ is a natural isomorphism. Since $\eta^{*}=\alpha^{*} \omega^{*}$, we see that $\omega^{*}$ is an isomorphism if and only if $\eta^{*}$ is.

If $\mathbf{A}-\mathrm{MOD} \rightarrow$ left $\mathbf{A} \uparrow-\mathrm{MOD}$ preserves enough injectives, then for each $\mathbf{N}$, $\operatorname{Ext}_{\mathbf{A} \uparrow}^{*}\left(\mathbf{N}\right.$ !, - !) will be a universal $\delta$-functor on A-MOD. Hence, $\eta^{*}$ will be an isomorphism. When $\mathbf{N}$ is projective, it follows that $\operatorname{Ext}_{\mathbf{A} \uparrow}^{n}(\mathbf{N}$ !, $\mathbf{M}$ !) vanishes for all $\mathbf{M}$ and $n>0$. Thus, Ext $t_{\mathbf{A} \uparrow}^{*}\left(-!, \mathbf{M}\right.$ !) is universal for each $\mathbf{M}$ and $\eta^{*}$ is a natural isomorphism of bifunctors. Now, in A-MOD there are enough relative injectives which are products of primitives $(\$ 2)$. Hence, the CCT will follow from: If $\mathbf{I}$ is a primitive relative injective in $\mathbf{A}-\mathrm{MOD}$, then $\mathbf{I}$ ! is a relative injective as a left A $\uparrow$-module.

The next section is devoted to proving this statement in the f.H.p. case.

13. Proof of the CCT in the f.H.p. case. Let $\Re$ and $\Re$ be left $\mathbf{A} \uparrow$-modules. Define $\mathcal{K}^{i j}$ to be $e^{i} \otimes e^{j} \mathcal{K}$, an $e^{i} \otimes e^{j} \mathbf{A} \uparrow$-module. Note that only the $\mathbf{A}^{i} \otimes \mathbf{A}^{j}$ piece of $e^{i} \otimes e^{j} \mathbf{A} \uparrow$ acts nontrivially on $\Re^{i j}$. For $\Re=\mathbf{N}$ !, $\Re^{i j}=\mathbf{N}^{i} \varphi^{i j}$ and, so, $\Re=$ $\Pi_{i \in I} \amalg_{j \geqslant i} \Re^{i j}$. If $g \in \operatorname{Hom}_{\mathbf{A} \uparrow}(\Re, \Re)$, then $g\left(\Re^{i j}\right) \subseteq \Re^{i j}$. When $\Re=\Pi_{i} \Pi_{j \geqslant i} \Re^{i j}$, one readily sees that $\operatorname{Hom}_{\mathbf{A} \uparrow}(\Re, \Re)$ is the subgroup of $\Pi_{i} \Pi_{j \geqslant i} \operatorname{Hom}_{\mathbf{A}^{i} \otimes \mathbf{A}^{j}}\left(\mathscr{T}^{i j}, \Re^{i j}\right)$ consisting of those $\left\{g^{i j}\right\}$ which satisfy:

$$
\begin{aligned}
& \text { for all } m \in \Re^{i j}, h \leqslant i, \text { and } k \geqslant j, \\
& g^{h k}\left(\varphi^{h i} \otimes \varphi^{j k} \cdot m\right)=\varphi^{h i} \otimes \varphi^{j k} g^{i j}(m) .
\end{aligned}
$$

This applies, in particular, for $\Re=\mathbf{N}$ ! - if $I_{p}$ is finite for all $p$. Consequently, we shall assume this throughout the remainder of this section. We further assume that the index set has a unique maximal element $\infty$, and that $\varphi^{i \infty}: \mathbf{A}^{\infty} \rightarrow \mathbf{A}^{i}$ is f.H.p. for every $i$.

From the results of the last section, we see that we need only show: if $I$ is a primitive relative injective in A-MOD, then $\operatorname{Hom}_{A \uparrow}(-, \mathbf{I} !)$ preserves the exactness of allowable exact sequences. For convenience, let 0 denote an arbitrary index, let $I$ be a relative injective in $\mathbf{A}^{0}$-MOD, and let $\mathbf{I}$ be given by $\mathbf{I}^{i}=I$ if $i \geqslant 0$, and $\mathbf{I}^{i}=0$ otherwise. (Note that $T^{i j}: \mathbf{I}^{j} \rightarrow \mathbf{I}^{i}$ is either the identity or 0 .) Of course, $\mathbf{I} !^{i j}=I \varphi^{i j}$ if $0 \leqslant i \leqslant j$, and is 0 otherwise. An element of $\operatorname{Hom}_{\mathbf{A} \uparrow}(\mathfrak{N}, \mathbf{I} !)$ is determined by a consistent tuple $\left\{g^{i j}\right\}$ for $i \geqslant 0$. We claim that there is a natural isomorphism

$$
\operatorname{Hom}_{\mathbf{A} \uparrow}(\mathfrak{R}, \mathbf{I} !) \rightarrow \operatorname{Home}_{\mathbf{A}^{0} \otimes \mathbf{A}^{\infty}}\left(\mathscr{T}^{0 \infty}, \mathbf{I} !^{0 \infty}\right) .
$$

First note, from (1), that if $g$ is in the former group, then $\varphi^{0 i} \otimes \varphi^{j \infty} g^{i j}(m)=$ $g^{0 \infty}\left(\varphi^{0 i} \otimes \varphi^{j \infty} m\right)$. But left multiplication by $\varphi^{0 i} \otimes \varphi^{j \infty}$ is a group isomorphism $I \varphi^{i j} \rightarrow I \varphi^{0 \infty}$. Hence, given any $g^{0 \infty} \in \operatorname{Hom}_{\mathbf{A}^{0} \otimes \mathbf{A}^{\infty}}\left(\mathscr{T}^{0 \infty}, \mathbf{I} !^{0 \infty}\right)$, the last equation will uniquely define a collection $\left\{g^{i j}\right\}$, which clearly satisfies (1). (The H.p. hypothesis is 
necessary to insure that $g^{i j}$ is an $\mathbf{A}^{j}$-morphism.) Thus, it suffices to show that $\mathbf{I} !^{0 \infty}=I \varphi^{0 \infty}$ is a relative injective left $\mathbf{A}^{0} \otimes \mathbf{A}^{\infty}$-module.

Let $M$ be a bimodule over an algebra $A$. If $\varphi: B \rightarrow A$ is an algebra morphism, use $\bar{M}$ to denote $M$ with the left $A \otimes B^{\text {op }}$-module structure: $a \otimes b \cdot x=a x \varphi(b)$. The CCT in the f.H.p. case follows from

Lemma. If $\varphi: B \rightarrow A$ gives $A$ the structure of a flat left $B$-module and $I$ is a (relative) injective $A$-bimodule, then $\bar{I}$ is a (relative) injective left $A \otimes B^{\mathrm{op}}$-module.

Proof. Let $M^{\prime} \rightarrow M$ be an (allowable) inclusion of $A \otimes B^{\mathrm{op}}$-modules and let $\theta$ : $M^{\prime} \rightarrow \bar{I}$ be any morphism. Then $\theta$ factors as

$$
M^{\prime} \stackrel{\theta_{1}^{\prime}}{\rightarrow} M^{\prime} \otimes_{B} A \stackrel{\theta_{2}}{\rightarrow} \bar{I} \otimes_{B} A \stackrel{\theta_{3}}{\rightarrow} I \stackrel{\theta_{4}}{\rightarrow} \bar{I}
$$

where $\theta_{1}^{\prime}(m)=m \otimes 1, \theta_{2}=\theta \otimes \mathrm{id}, \theta_{3}(x \otimes a)=x a$, and $\theta_{4}=$ id. Note that $\theta_{3} \theta_{2}$ is an $A$-bimodule morphism. Since $A$ is flat, there is an (allowable) $A$-bimodule inclusion $M^{\prime} \otimes_{B} A \rightarrow M \otimes_{B} A$. Then $\theta_{3} \theta_{2}$ can be extended to give $\hat{\theta}: M \otimes_{B} A \rightarrow I$. Let $\theta_{1}$ : $M \rightarrow M \otimes_{B} A$ be $\theta_{1}(m)=m \otimes 1$. Then the required extension of $\theta$ is $\theta_{4} \hat{\theta} \theta_{1}$.

14. Proof of the CCT in the $k$-f.H.p. case. We continue the assumption that $I_{p}$ is finite for all $p$ and replace the other assumptions of $\S 13$ by $k \rightarrow \mathbf{A}_{i}$ is f.H.p. for all $i$. If $\omega^{*}$ is a natural isomorphism of bifunctors on A-MOD we shall simply say " $\omega$ * is an isomorphism for A."

Let A: $I \rightarrow$ ALG be a diagram and let $m$ be a maximal (but not necessarily

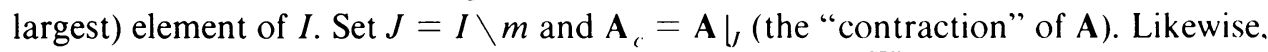
set $\mathbf{M}_{c}=\mathbf{M} \bigsqcup_{b}$ for any $\mathbf{M} \in \mathbf{A}$-MOD. An $\mathbf{A}_{c}$-bimodule $\overline{\mathbf{M}}$ can be extended to an A-bimodule $\overline{\mathbf{M}}_{+}$by setting $\overline{\mathbf{M}}_{+}^{m}=0$. Let $\bar{\pi}$ be an $\mathbf{A}_{c} \uparrow$-module and, for each $i<m$.

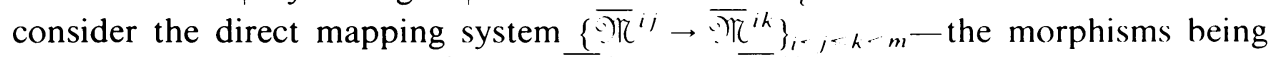
left multiplication by $e^{i} \otimes \varphi^{j k}$. Then $\overline{M r}_{+}^{i m}=\lim \bar{N}^{i j}{ }^{i j}$ has a natural structure as a left $\mathbf{A}^{i} \otimes \mathbf{A}^{m}$ module. If $i \nless m$, let $\bar{\Re}_{+}^{i m}=0$ and define an $\mathbf{A} \uparrow$-module as $\overline{\eta_{+}}=$ $\overline{\mathfrak{M}} \times \Pi_{i} \overline{\mathfrak{M}}_{+}^{i m}$. One then has $\overline{\mathbf{M}}_{+} !=\overline{\mathbf{M}} !_{+}$for any $\overline{\mathbf{M}} \in \mathbf{A}_{-}-$MOD. The extension functors $+: \mathbf{A}_{c}-$ MOD $\rightarrow \mathbf{A}$-MOD and $+: \mathbf{A}_{c} \uparrow$-left modules $\rightarrow \mathbf{A} \uparrow$-left modules are both full exact embeddings.

As remarked in $\$ 11$, a $k$-f.H.p. diagram can be extended to an f.H.p. one, for which we know that $\omega^{*}$ is an isomorphism. The problem here is to prove from this that it is an isomorphism for the original diagram. Taking the extended diagram as $A$, this is a special case of the following

THEOREM. Suppose that $\omega^{*}$ is an isomorphism for a diagram A: $I \rightarrow$ ALG. Let $m$ be any maximal element of $I$ and let $J=I \backslash\{m\}$. Then $\omega^{*}$ is an isomorphism for $\mathbf{A}_{c}=\left.\mathbf{A}\right|_{J}$.

Proof. The hypothesis is equivalent to: $\eta_{\mathbf{A}}^{*}: \operatorname{Ext}_{\mathbf{A}}^{*}(-,-) \rightarrow \operatorname{Ext}_{\mathbf{A}}^{*}(-!,-!)$ is an isomorphism. It will suffice to show the same is true of

$$
\eta^{*}: \operatorname{Ext}_{\mathbf{A}_{c}}^{*}(-,-) \rightarrow \operatorname{Ext}_{\mathbf{A}_{c t}}^{*}(-!,-!) .
$$


(i) (epi). Let $8: 0 \rightarrow \mathbf{M} ! \rightarrow \mathcal{O}_{n-1} \rightarrow \cdots \rightarrow \mathrm{O}_{1} \rightarrow \mathbf{N} ! \rightarrow 0$ represent a class in $\operatorname{Ext}_{\mathbf{A}_{,},}^{n}(\mathbf{N} !, \mathbf{M} !)$. Then, since $\eta_{\mathbf{A}}^{n}$ is an epimorphism and $\mathbf{M} !_{+}=\mathbf{M}_{+} !, \varepsilon_{+}$is equivalent to some $E$ !, where $E: 0 \rightarrow \mathbf{M} \rightarrow \mathbf{M}_{n-1} \rightarrow \cdots \rightarrow \mathbf{M}_{1} \rightarrow \mathbf{N} \rightarrow 0$ is in A-MOD. So $\stackrel{s}{=} \bar{E}_{+c}$ is equivalent to $E_{c} !$

(ii) (mono). Let $E$ represent a class in $\operatorname{Ext}_{\mathbf{A}_{r}}^{n}(\mathbf{N}, \mathbf{M})$. If $E$ ! is equivalent to 0 , then so is $E !_{+}$. But $E !_{+}=E_{+}$! and $\eta_{\mathrm{A}}^{n}$ is a monomorphism. Hence, $E_{+}$is equivalent to 0 . Then $E=E_{+c}$ represents the trivial class as well.

The $k$-f.H.p. case is a kind of "affine" case of the CCT. For if we have an affine variety, let $k$ be its function ring, and $\mathbf{A}^{i}$ the localizations corresponding to the basic opens. In this way, one obtains a $k$-f.H.p. diagram. (Since $k$ will be noetherian, the finiteness hypotheses will follow.)

15. Projective complexes over elementary modules. An A-bimodule $\mathbf{N}$ will be called q-elementary if $\mathbf{N}^{i}=0$ for $i \neq q$ (i.e., $\mathbf{N}$ is in the image of the functor $F^{q}$ ). In this section, we construct and compare projective complexes over $\mathbf{N}$ and $\mathbf{N}$ ! for elementary modules $\mathbf{N}$. While these complexes are always allowable resolutions, we postpone the proof of this to the next section. They will then be used to prove that $\omega^{*}: \operatorname{Ext}_{\mathbf{A}}^{*}(\mathbf{N},-) \rightarrow \operatorname{Ext}^{*}{ }_{\mathbf{A} !}(\mathbf{N} !,-!)$ is an isomorphism whenever $\mathbf{N}$ is elementary. Finally, an induction will deliver the CCT in the finite case.

To set the context for the eventual comparison of complexes, let $\mathbb{Q}$ be an abelian category with a distinquished class of allowable morphisms and enough relative projectives [M, Chapter XII]. Let $A$ be an object in $\mathbb{Q}$ and choose an arbitrary allowable (relative) projective resolution $X$. of $A: \cdots \rightarrow X_{n+1} \rightarrow X_{n} \rightarrow \cdots \rightarrow X_{0} \rightarrow$ $A \rightarrow 0$. [That is, each $X_{n}$ is a relative projective and every morphism is allowable.] Then $\operatorname{Ext}_{\mathscr{*}}^{*}(A,-)$ is naturally isomorphic to $H^{*}\left(\operatorname{Hom}_{\mathbb{Q}}(X .,-)\right)$ [M, p.269].

Let $\mathbf{N}$ be an object in A-MOD and choose arbitrary allowable (relative) projective resolutions $X . \rightarrow \mathbf{N} \rightarrow 0$ and $Y . \rightarrow \mathbf{N} ! \rightarrow 0$. Since $!$ is exact, $X . ! \rightarrow \mathbf{N} ! \rightarrow 0$ is a resolu-

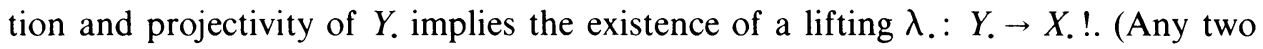
such liftings are chain homotopic.) Thus, there is a map of complexes

$$
\operatorname{Hom}_{\mathbf{A} !}(X . !,-) \rightarrow \operatorname{Hom}_{\mathbf{A} !}(Y . !,-) .
$$

Composing (1) with the isomorphism $\operatorname{Hom}_{\mathbf{A}}(-,-) \rightarrow \operatorname{Hom}_{\mathbf{A} !}(-!,-!)$ and invoking the description of Ext above produces

$$
\operatorname{Ext}_{\mathbf{A}}^{*}(\mathbf{N},-) \rightarrow \operatorname{Ext}_{\mathbf{A} !}^{*}(\mathbf{N} !,-!),
$$

which is independent of the lifting $\lambda$. . The universality of $\operatorname{Ext}_{\mathbf{A}}^{*}(-,-)$ now guarantees that (2) coincides with $\omega^{*}$. Similar remarks apply with $\mathbf{A}$ ! replaced by $\mathbf{A} \uparrow$ and $\omega^{*}$ by $\eta^{*}$.

Suppose now that $\mathbf{N}$ is $q$-elementary. Let $\Sigma=\Sigma^{q}=\Sigma\left(I^{q}\right)$, where $I^{q}$ is the ideal $I^{q}=\{i \mid i<q\} \subset I$. Set $\Sigma_{-1}=\{(q)\}$ and extend the definition of the boundary $\partial$ to the 0 -chains by $\partial(i)=(q)$. The projective complex over $\mathbf{N}$ will be built from one over $F^{q}\left(\mathbf{A}^{q e}\right)$, the $q$-elementary bimodule determined by $\mathbf{A}^{q e}$.

For $i \in I$, let $\mathbf{P}(i)$ be the primitive (relative) projective given by $\mathbf{P}(i)^{j}=\mathbf{A}^{j e}$ if $j \leqslant i$, and $\mathbf{P}(i)^{j}=0$ otherwise. If $i \geqslant k \geqslant j$, then the internal morphism $\mathbf{A}^{k e} \rightarrow \mathbf{A}^{j e}$ of 
$\mathbf{P}(i)$ is just $\varphi^{j k} \otimes \varphi^{j k}$. For every $\sigma \in \Sigma$, let $\mathbf{P}(\sigma)$ denote a copy of $\mathbf{P}(v \sigma)$ indexed by $\boldsymbol{\sigma}$ and set $\mathbf{P}_{n}=\amalg_{\sigma \in \Sigma_{n-1}} \mathbf{P}(\sigma)$. Note that $\mathbf{P}_{1}$ is just the coproduct of all $\mathbf{P}(i)$ with $i \in I^{q}$ and $\mathbf{P}_{0}=\mathbf{P}(q)$. The boundary $\partial_{n+1}: \mathbf{P}_{n+1} \rightarrow \mathbf{P}_{n}$ is determined by its restrictions to $\mathbf{P}(\sigma)$ for $\sigma \in \Sigma_{n}$. Let $a \in \mathbf{P}(\sigma)^{l}$ for some $l \leqslant v \sigma$. Then $\partial_{n+1}^{l} a=\left(a_{\tau}\right) \in \mathbf{P}_{n}^{l}=$ $\amalg_{\Sigma_{n-1}} \mathbf{P}(\tau)^{l}$, where $a_{\tau}=(-1)^{n-r} a$ if $\tau=\sigma_{r}$ for some $r$, and $a_{\tau}=0$ otherwise. (Notice that this is meaningful since $v \sigma_{r} \geqslant v \sigma$ for every $r$ and, so, $\mathbf{P}\left(\sigma_{r}\right)^{l}=\mathbf{P}(\sigma)^{l}$.) It is easy to verify that $\partial_{n+1}$ is a morphism and that $\partial_{n} \partial_{n+1}=0$ for $n \geqslant 1$. There is an allowable epimorphism $\varepsilon: \mathbf{P}_{0} \rightarrow F^{q}\left(A^{q e}\right)$ defined by $\varepsilon^{q}=\mathrm{id}$ and $\varepsilon^{i}=0$ for $i \neq q$. It is immediate that $\varepsilon$ is a cokernel of $\partial_{1}$, and, so, we have a projective complex

$$
\text { P. } \rightarrow F^{q}\left(\mathbf{A}^{q e}\right) \rightarrow 0 \text {. }
$$

Before describing the projective complex over $\mathbf{N}$, we turn to that over

$$
F^{q}\left(\mathbf{A}^{q e}\right) !=\coprod_{k \geqslant q} \mathbf{A}^{q} \otimes \mathbf{A}^{q} \varphi^{q k} .
$$

For $i \leqslant q$, set

$$
\mathscr{P}(i)=\mathbf{A} \uparrow e^{i} \otimes e^{q}=\prod_{h \leqslant i} \coprod_{k \geqslant q} \mathbf{A}^{h} \varphi^{h i} \otimes \mathbf{A}^{q} \varphi^{q k} .
$$

This is a relative projective left $\mathbf{A} \uparrow$-module, as it is generated by an idempotent. As before, we define $\mathscr{P}(\sigma)$ and $\mathscr{P}_{n}$ to be $\mathcal{P}(v \sigma)$ and $\amalg_{\sigma \in \Sigma_{n-1}} \mathscr{P}(\sigma)$, respectively. Again, the boundary $\partial_{n+1}: \mathscr{P}_{n+1} \rightarrow \mathscr{P}_{n}$ is determined by its restrictions $\partial_{n+1}^{\sigma}$ to $\mathscr{P}(\sigma)$ for $\sigma \in \Sigma_{n}$. Recall that $\varphi(i, j)=\varphi^{i j}$ for $i \leqslant j$ and $\varphi(\sigma, \tau)=\varphi(v \sigma, v \tau)$ whenever $v \sigma \leqslant$ $v \tau$. Then define the $\mathbf{A} \uparrow$-morphism $\partial_{n+1}^{\sigma}$ by

$$
\partial_{n+1}^{\sigma} a e^{v \sigma} \otimes e^{q}=\left(a_{\tau}\right) \in \mathscr{P}_{n}=\coprod_{\Sigma_{n-1}} \mathscr{P}(\tau),
$$

where $a_{\tau}=(-1)^{n-r} a \varphi\left(\sigma, \sigma_{r}\right) \otimes e^{q}$ if $\tau=\sigma_{r}$ for some $r$, and $a_{\tau}=0$ otherwise. Clearly, $\partial_{n} \partial_{n+1}=0$ for $n \geqslant 1$. The image of $\partial_{1}$ in $\mathscr{P}_{0}=\mathbf{A} \uparrow e^{q} \otimes e^{q}$ is $\prod_{h<q} \amalg_{k \geqslant q} \mathbf{A}^{h} \varphi^{h q} \otimes \mathbf{A}^{q} \varphi^{q k}$. So the quotient morphism $\varepsilon^{\prime}: \mathscr{P}_{0} \rightarrow F^{q}\left(\mathbf{A}^{q e}\right)$ ! is a cokernel of $\partial_{1}$ and is allowable. We now have a projective complex

$$
\text { P. } \rightarrow F^{q}\left(\mathbf{A}^{q e}\right) ! \rightarrow 0 .
$$

Even though we have not yet shown (3) to be an allowable resolution, we construct an explicit comparison morphism $\lambda .: \mathscr{P}_{.} \rightarrow$ P. !. We begin by defining, for each $i \leqslant q$, an $\mathbf{A} \uparrow$-morphism $\lambda^{i}: \mathscr{P}(i) \rightarrow \mathbf{P}(i)$ !. Now $\lambda^{i}$ is determined by the destination of $e^{i} \otimes e^{q}$ in $\mathbf{P}(i) !$. Set $\lambda^{i}\left(e^{i} \otimes e^{q}\right)=e^{i} \otimes e^{i} \varphi^{i q}$. Since the morphism $\mathbf{A}^{i e} \rightarrow \mathbf{A}^{h e}$ is just $\varphi^{h i} \otimes \varphi^{h i}$, we see that the product of $\varphi^{h i} \otimes e^{q} \in \mathbf{A} \uparrow$ with $e^{i} \otimes e^{i} \varphi^{i q} \in \mathbf{P}(i)$ ! is just $e^{h} \otimes e^{h} \varphi^{h q} \in \mathbf{P}(i)$ ! and, consequently, $\lambda^{i}\left(a^{h} \varphi^{h i} \otimes b^{q} \varphi^{q k}\right)=a^{h} \otimes \varphi^{h q}\left(b^{q}\right) \varphi^{h k}$. Next, let $\lambda^{\sigma}: \mathcal{P}(\sigma) \rightarrow \mathbf{P}(\sigma)$ ! be $\lambda^{v \sigma}$ and define $\lambda_{n}$ to be $\lambda_{n}=\amalg_{\sigma \in \Sigma_{n-1}} \lambda^{\sigma}$. It is easy to verify that $\lambda$. is a chain map.

After assembling some facts and notation, we shall turn our attention to an arbitrary $q$-elementary bimodule $\mathbf{N}$. If $\Omega$ is any $k$-algebra and $V$ is a left $\Omega$-module, then $\Omega \otimes|V|$ is a relative left $\Omega$-projective, since $\Omega \otimes-$ is the left adjoint to an obvious forgetful functor $|-|$. Then multiplication, $\Omega \otimes|V| \rightarrow V$, is an allowable 
epimorphism. (The $k$-splitting is $v \mapsto 1 \otimes v$.) We define $\Omega$-modules $K_{n}$ inductively by $K_{0}=V$ and

$$
0 \rightarrow K_{n} \rightarrow \Omega \otimes\left|K_{n-1}\right| \rightarrow K_{n-1} \rightarrow 0 \text { for } n>0 .
$$

(At each stage, we select a specific kernel.) Although we shall not need it here, we remark that splicing these short exact sequences yields an allowable relative projective resolution of $V$.

When $\mathbf{M}$ is an A-bimodule and $W$ is a $k$-module, $\mathbf{M} \otimes W$ will be the bimodule given by $(\mathbf{M} \otimes W)^{i}=\mathbf{M}^{i} \otimes W$. Note that $F^{q}(M) \otimes W=F^{q}(M \otimes W)$ for any $\mathbf{A}^{q}$-bimodule $M$. If $\mathbf{M}$ is a $j$-primitive relative projective, then $\mathbf{M} \otimes W$ is also.

For each $r \geqslant 1$, let $\mathbf{Q}_{r}$ be a kernel of $\partial_{r-1}: \mathbf{P}_{r-1} \rightarrow \mathbf{P}_{r-2}$. So $\mathbf{Q}_{1}=\operatorname{ker} \varepsilon$. Let $M$ be an arbitrary $\mathbf{A}^{q}$-bimodule. Since $\varepsilon$ is allowable, tensoring with $|\boldsymbol{M}|$ produces an allowable exact sequence

$$
0 \rightarrow \mathbf{Q}_{1} \otimes|M| \rightarrow \mathbf{P}_{0} \otimes|M| \rightarrow F^{q}\left(\mathbf{A}^{q e}\right) \otimes|M| \rightarrow 0 .
$$

Composing the last morphism with the allowable epimorphism $F^{q}\left(\mathbf{A}^{q e} \otimes|M|\right) \rightarrow$ $F^{q}(M)$ yields an allowable epimorphism $\varepsilon_{M}: \mathbf{P}_{0} \otimes|M| \rightarrow F^{q}(M)$. We now apply (5) with $\Omega=\mathbf{A}^{q e}$ and $V=N$. For simplicity, we abbreviate $\varepsilon_{K_{n}}$ and $\mathrm{id}_{K_{n}}$ to $\varepsilon_{n}$ and id $\mathrm{id}_{n}$. We define $\mathbf{L}_{n}$ to be a kernel of $\varepsilon_{n-1}$ when $n \geqslant 1$. Since $F^{q}$ is exact, there is a commutative diagram of exact sequences:

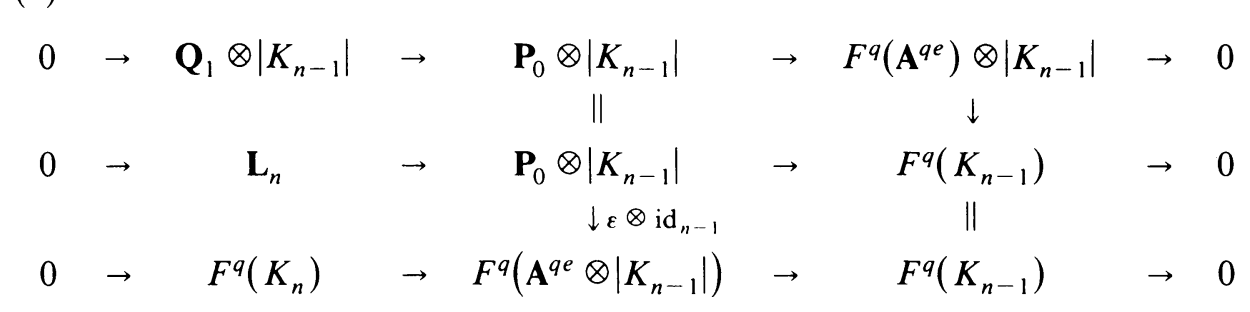

There are canonical morphisms completing the left column of (7) to an allowable short exact sequence. Note that we have a morphism $\partial_{1} \otimes \mathrm{id}_{n-1}: \mathbf{P}_{1} \otimes\left|K_{n-1}\right| \rightarrow$ $\mathbf{Q}_{1} \otimes\left|K_{n-1}\right|$. Also, since $\mathbf{P}_{0} \otimes\left|K_{n}\right|$ is a relative projective and $\varepsilon \otimes \mathrm{id}_{n-1}: \mathbf{L}_{n} \rightarrow$ $F^{q}\left(K_{n}\right)$ is an allowable epimorphism, $\varepsilon_{n}$ lifts to give $\hat{\varepsilon}_{n}: \mathbf{P}_{0} \otimes\left|K_{n}\right| \rightarrow \mathbf{L}_{n}$. (There will be many choices for $\hat{\varepsilon}_{n}$. We fix one for the remainder of this discussion.) Thus, continuing the use of matrix notation for morphisms, $\left(\partial_{1} \otimes \mathrm{id}_{n-1} \hat{\varepsilon}_{n}\right)$ is a morphism $\mathbf{P}_{1} \otimes\left|K_{n-1}\right| \oplus \mathbf{P}_{0} \otimes\left|K_{n}\right| \rightarrow \mathbf{L}_{n}$. Now, for each $i$ there is a $k$-splitting $\mathbf{L}_{n}^{i}=\mathbf{Q}_{1}^{i} \otimes$ $\left|K_{n-1}\right| \oplus V^{i}$ with $\rho^{i}: F^{q}\left(K_{n}\right)^{i} \stackrel{\sim}{\rightarrow} V^{i}$. So $\operatorname{im}\left(\hat{\varepsilon}_{n}^{i}-\rho^{i} \varepsilon^{i}\right) \subseteq \mathbf{Q}_{1}^{i} \otimes\left|K_{n-1}\right|$. But im $\rho^{i} \cap \mathbf{Q}_{1}^{i}$ $\otimes\left|K_{n-1}\right|=(0)$, and, hence, im $\hat{\varepsilon}_{n}^{i} \cap \mathbf{Q}_{1}^{i} \otimes\left|K_{n-1}\right|=(0)$. This implies that $\hat{\varepsilon}_{n}$ is allowable and that

$$
\operatorname{ker}\left(\partial_{1} \otimes \mathrm{id}_{n-1} \hat{\varepsilon}_{n}\right)=\operatorname{ker}\left(\partial_{1} \otimes \mathrm{id}_{n-1}\right) \oplus \operatorname{ker} \varepsilon_{n}=\operatorname{ker}\left(\partial_{1} \otimes \mathrm{id}_{n-1}\right) \oplus \mathbf{L}_{n+1}
$$

Furthermore, if $\partial_{1}$ is allowable and (3) is exact at $\mathbf{P}_{0}$, then $\left(\partial_{1} \otimes \mathrm{id}_{n-1} \hat{\varepsilon}_{n}\right)$ is an allowable epimorphism.

We can now describe the projective complex over $\mathbf{N}=F^{q}(N)$. Define $\mathbf{P}_{n}(N)$ by

$$
\mathbf{P}_{n}(N)=\coprod_{i=0}^{n} \mathbf{P}_{i} \otimes\left|K_{n-1}\right| .
$$


The boundary $\partial_{n+1}(N): \mathbf{P}_{n+1}(N) \rightarrow \mathbf{P}_{n}(N)$ is determined by its restrictions to $\mathbf{P}_{i} \otimes\left|K_{n+1-i}\right|$. For $i \geqslant 1$,

$$
\partial_{n+1}(N)=\partial_{i} \otimes \mathrm{id}_{n+1-i}: \mathbf{P}_{i} \otimes\left|K_{n+1-i}\right| \rightarrow \mathbf{P}_{i-1} \otimes\left|K_{n+1-i}\right| .
$$

For $i=0, \partial_{n+1}(N)=\hat{\varepsilon}_{n+1}$. (We abuse notation by using $\hat{\varepsilon}_{n}$ to represent $\mathbf{P}_{0} \otimes\left|K_{n}\right| \rightarrow$ $\mathbf{L}_{n} \rightarrow \mathbf{P}_{0} \otimes\left|K_{n-1}\right|$.) Note that $\partial_{n+1}(N)$ is allowable if every $\partial_{i}$ is. Also, we see that

$$
\operatorname{ker} \partial_{n}(N)=\coprod_{i \geqslant 1} \operatorname{ker}\left(\partial_{i} \otimes \operatorname{id}_{n-i}\right) \oplus \mathbf{L}_{n+1} .
$$

Further, $\partial_{n+1}(N)$ factors through $\amalg_{i \geqslant 2} \mathbf{Q}_{i} \otimes\left|K_{n+1-i}\right| \oplus \mathbf{L}_{n+1}$. Thus, $\partial_{n}(N) \partial_{n+1}(N)$ $=0$ and we have a (relative) projective complex

$$
\mathbf{P} .(N) \rightarrow \mathbf{N} \rightarrow 0 \quad \text { with } \mathbf{P}_{n}(N)=\coprod \mathbf{P}_{i} \otimes\left|K_{n-i}\right| .
$$

When (3) is an allowable resolution,

$$
\operatorname{ker}\left(\partial_{i} \otimes \mathrm{id}_{n-i}\right)=\mathbf{Q}_{i+1} \otimes\left|K_{n-i}\right| \quad \text { and } \quad \operatorname{im} \partial_{n+1}(N)=\coprod_{i \geqslant 2} \mathbf{Q}_{i} \otimes\left|K_{n+1-i}\right| \oplus \mathbf{L}_{n+1} \text {. }
$$

So (8) is an allowable resolution as well. Finally, note that this complex is not functorial in $N$. It depends upon the choices of the $K_{n}$ and $\hat{\varepsilon}_{n}$. (The first of these problems can be surmounted since the categories are selective [M,p. 256]. The second, however, seems inescapable.)

Naturally, we now wish to describe a projective complex over N!. We make two preliminary observations. First, if $\mathbf{M}$ is an $\mathbf{A}$-bimodule and $W$ is a $k$-module, then $(\mathbf{M} \otimes W) !=\mathbf{M} ! \otimes W$. Second, for an arbitrary $\mathbf{A}^{q}$-bimodule $M$, we have an allowable epimorphism $F^{q}\left(\mathbf{A}^{q e} \otimes|M|\right) ! \rightarrow F^{q}(M)$ !. We can now proceed as before-with a slight twist. We let $Q_{r}$ be the kernel of $\partial_{r-1}: P_{r-1} \rightarrow P_{r-2}$ and define $\varepsilon_{M}^{\prime}$ to be the allowable epimorphism $\mathscr{P}_{0} \otimes|M| \rightarrow F^{q}\left(\mathbf{A}^{q e}\right) ! \otimes|M| \rightarrow F^{q}(M)$ !. Note that $\varepsilon_{M}^{\prime}=$ $\varepsilon_{M} !\left(\lambda_{0} \otimes \mathrm{id}_{M}\right)$. Again, for all $n \geqslant 1$, define $L_{n}$ as $L_{n}=\operatorname{ker} \varepsilon_{n-1}^{\prime}$. This time we must exercise some care in our choice of the liftings $\hat{\varepsilon}_{n}^{\prime}$, as follows:

$$
\left(\varepsilon ! \otimes \mathrm{id}_{n-1}\right)\left(\lambda_{0} \otimes \mathrm{id}_{n-1}\right)=\varepsilon^{\prime} \otimes \mathrm{id}_{n-1}
$$

and, consequently, the restrictions to $L_{n}$ coincide. Also, $\varepsilon_{n} !\left(\lambda_{0} \otimes \mathrm{id}_{n}\right)=\varepsilon_{n}^{\prime}$. Thus, $\hat{\varepsilon}_{n} !\left(\lambda_{0} \otimes \mathrm{id}_{n}\right)$ is a lifting of $\varepsilon_{n}^{\prime}$ to a morphism $\mathscr{P}_{0} \otimes\left|K_{n}\right| \rightarrow \mathbf{L}_{n}$ ! whose image is contained in the image of $\lambda_{0} \otimes \mathrm{id}_{n-1}: L_{n} \rightarrow \mathbf{L}_{n}$ !. This can then be lifted further to give $\varepsilon_{n}^{\prime}: \mathscr{P}_{0} \otimes\left|K_{n}\right| \rightarrow L_{n}$ satisfying $\left(\lambda_{0} \otimes \mathrm{id}_{n-1}\right) \hat{\varepsilon}_{n}^{\prime}=\hat{\varepsilon}_{n} !\left(\lambda_{0} \otimes \mathrm{id}_{n}\right)$. Now, with these choices for $\hat{\varepsilon}_{n}^{\prime}$, we proceed precisely as before and obtain a projective complex

$$
\mathscr{P}_{.}(N) \rightarrow \mathbf{N} ! \rightarrow 0 \quad \text { with } \mathscr{P}_{n}(N)=\coprod \mathscr{P}_{i} \otimes\left|K_{n-i}\right| .
$$

Naturally, on $\mathscr{P}_{i} \otimes\left|K_{n+1-i}\right|$ for $i \geqslant 1$, the boundary is $\partial_{n+1}(N)=\partial_{i} \otimes \mathrm{id}_{n+1-i}$; for $i=0, \partial_{n+1}(N)=\hat{\varepsilon}_{n+1}^{\prime}$. When (4) is an allowable resolution, (9) is as well.

Finally, we need the comparison morphism $\lambda .(N)$ : $\mathcal{P}_{.}(N) \rightarrow$ P. $(N)$ !. Since $\mathbf{P}_{n}(N) !=\amalg \mathbf{P}_{i} ! \otimes\left|K_{n-i}\right|$, it is not surprising that we define $\lambda_{n}(N)$ by

$$
\lambda_{n}(N)=\coprod_{i \leqslant n} \lambda_{i} \otimes \mathrm{id}_{n-i} .
$$

This will give a chain map if $\lambda_{i-1}(N) \partial_{i}(N)=\partial_{i}(N) ! \lambda_{i}(N)$ for all $i \geqslant 1$. (We have already noted that $\varepsilon_{N}^{\prime}=\varepsilon_{N} ! \lambda_{0}(N)$.) Since $\lambda$. is itself a chain map, we need only show 
$\hat{\varepsilon}_{n} !\left(\lambda_{0} \otimes \mathrm{id}_{n}\right)=\left(\lambda_{0} \otimes \mathrm{id}_{n-1}\right) \hat{\varepsilon}_{n}^{\prime}$. But our choices for the liftings $\hat{\varepsilon}_{n}^{\prime}$ were designed to satisfy this equation and, so, $\lambda .(N)$ is a chain map.

16. A restricted CCT and the CCT for finite diagrams. We wish to show that the relative projective complexes constructed in the last section are allowable resolutions. As we have remarked, it suffices to show this for (3) and (4) of that section. Once again, we make some preliminary observations.

If $J$ is a partially ordered set with a smallest element 0 , then $\Sigma(J)$ is the cone on $\Sigma(J \backslash\{0\})$. Consequently, it is contractible and its chain complex is exact. Denote the chain complex by

$$
\cdots \rightarrow C_{n}(J) \stackrel{\partial_{n}}{\rightarrow} \cdots \rightarrow C_{1}(J) \stackrel{\partial_{1}}{\rightarrow} C_{0}(J) \stackrel{\varepsilon}{\rightarrow} \mathbf{Z} \rightarrow 0 .
$$

Recall that $C_{n}(J)$ is the free abelian group generated by $\Sigma_{n}(J)$. So tensoring (1) with any ring will result in another exact sequence. We note for future use that (1) has a contracting homotopy: for $n \geqslant 0$, define $\mathcal{K}_{n}: C_{n}(J) \rightarrow C_{n+1}(J)$ by

$$
\mathscr{K}_{n}\left(i_{n}<\cdots<i_{0}\right)=(-1)^{n}\left(0<i_{n}<\cdots<i_{0}\right)
$$

if $i_{n} \neq 0$ and $\mathcal{K}_{n}\left(0<\cdots<i_{0}\right)=0$. Also, define $\mathcal{K}_{-1}: \mathbf{Z} \rightarrow C_{0}(J)$ by $\mathcal{K}_{-1} 1=(0)$. One easily checks the conditions for a contracting homotopy, to wit: $\varepsilon \mathscr{K}_{-1}=\mathrm{id}$, $\partial_{1} \mathcal{K}_{0}+\mathcal{K}_{-1} \varepsilon=\mathrm{id}$, and $\partial_{n+1} \mathcal{K}_{n}+\mathcal{K}_{n-1} \partial_{n}=$ id for $n>0$.

Let $\Theta$ and $\Re$ be abelian categories, and let $F: \Theta \rightarrow$ $\mathbb{R}$ be an exact, additive, faithful functor. [Examples. (i) $Q=A-M O D, ~ \Re=k$-MOD, $F=U$ (as defined in §7); (ii) $\mathbb{Q}=\Omega$-left mod, $\mathfrak{N}=k$-MOD, $F=|-|$.] Suppose that $X$. $\rightarrow A$ is a complex in $Q$. Then it is an $F$-allowable resolution if and only if $F(X$.) $\rightarrow F(A)$ has a contracting homotopy [M, p. 265].

Now consider the complex $\mathbf{P} . \rightarrow F^{q}\left(\mathbf{A}^{q e}\right) \rightarrow 0$. For any $\mathbf{A}$-bimodule $\mathbf{M}, U(\mathbf{M})=$ $\Pi\left|M^{i}\right|$. We need a contracting homotopy for the complex $U(\mathbf{P}.) \rightarrow U\left(F^{q}\left(\mathbf{A}^{q e}\right)\right) \rightarrow 0$ in $k$-MOD. But this complex is just the product of the complexes

$$
\left|\mathbf{P}^{p}\right| \rightarrow\left|F^{q}\left(\mathbf{A}^{q e}\right)^{p}\right| \rightarrow 0 .
$$

It then suffices to exhibit the contracting homotopy for each of these. Since $\mathbf{P}(\sigma)^{p}=\mathbf{P}(v \sigma)^{p}=0$, if $p \$ v \sigma,(2)$ is the zero complex if $p \notin I^{q}$. When $p=q$, (2) reduces to $0 \rightarrow\left|\mathbf{A}^{q e}\right| \rightarrow\left|\mathbf{A}^{q e}\right| \rightarrow 0$ and the identity is a contracting homotopy. Finally, if $p<q$, let $J=I^{q} \cap I_{p}=\{i \mid p \leqslant i<q\}$ and $\Sigma^{\prime}=\Sigma(J)$. [Note that $p$ is the smallest element of $J$.] Then

$$
\mathbf{P}_{n}^{p}=\coprod_{\sigma \in \Sigma_{n-1}^{\prime}} \mathbf{P}(\boldsymbol{\sigma})^{p}=\mathbf{A}^{p e} \otimes C_{n-1}(J), \quad \mathbf{P}_{0}^{p}=\mathbf{A}^{p e}, \quad F^{q}\left(\mathbf{A}^{q e}\right)^{p}=0,
$$

and (2) reduces to $\left|\mathbf{A}^{p e}\right| \otimes C .(J) \rightarrow\left|\mathbf{A}^{p e}\right| \rightarrow 0$. Since this is also the result of tensoring (1) with $\mathbf{A}^{p e}$ and applying $|-|$, the required homotopy is $\operatorname{id}_{\mathbf{A}^{p e}} \otimes \mathcal{K}_{.}$.

Next, we examine $\mathscr{P}_{\text {. }} \rightarrow F^{q}\left(\mathbf{A}^{q e}\right) ! \rightarrow 0$. For each $p \leqslant q$, let $E_{p} \in \mathbf{A} \uparrow$ be the idempotent $E_{p}=\sum_{j \geqslant q} e^{p} \otimes e^{j}$. Observe that

$$
E_{p} \mathscr{P}(i)= \begin{cases}\coprod_{k \geqslant q} \mathbf{A}^{p} \varphi^{p i} \otimes \mathbf{A}^{q} \varphi^{q k} & \text { if } p \leqslant i, \\ 0 & \text { otherwise. }\end{cases}
$$


Hence, for any $i \leqslant q$,

$$
\mathscr{P}(i)=\left(\sum_{p \leqslant q} E_{p}\right) \mathscr{P}(i)=\prod_{p \leqslant q} E_{p} \mathscr{P}(i)
$$

and, so, $\mathscr{P}_{n}=\prod_{p \leqslant q} E_{p} \mathscr{P}_{n}$. Likewise, $E_{p} F^{q}\left(\mathbf{A}^{q e}\right) !$ is $F^{q}\left(\mathbf{A}^{q e}\right)$ ! when $p=q$, and is 0 otherwise. So, as before, it suffices to exhibit a contracting homotopy for each of the complexes

$$
\left|E_{p} \mathscr{P} .\right| \rightarrow\left|E_{p} F^{q}\left(\mathbf{A}^{q e}\right) !\right| \rightarrow 0 .
$$

When $p=q,(3)$ reduces to

$$
0 \rightarrow\left|\coprod_{k \geqslant q} \mathbf{A}^{q} \otimes \mathbf{A}^{q} \varphi^{q k}\right| \rightarrow\left|F^{q}\left(\mathbf{A}^{q e}\right) !\right| \rightarrow 0,
$$

and the identity is a contracting homotopy. If $p<q$, let $J=I_{p} \cap I^{q}$ and $\Sigma^{\prime}=\Sigma(J)$. Then $\left|E_{p} \mathscr{P}_{n}\right|$ is isomorphic as a $k$-module to $\left|\amalg_{k \geqslant q} \mathbf{A}^{p} \otimes \mathbf{A}^{q} \varphi^{q k}\right| \otimes C_{n-1}(J)$. Now

$$
F^{q}\left(\mathbf{A}^{p} \otimes \mathbf{A}^{q}\right) !=\coprod_{k \geqslant q} \mathbf{A}^{p} \otimes \mathbf{A}^{q} \varphi^{q k} .
$$

We see that (3) reduces to

$$
\left|F^{q}\left(\mathbf{A}^{p} \otimes \mathbf{A}^{q}\right) !\right| \otimes C .(J) \rightarrow\left|F^{q}\left(\mathbf{A}^{p} \otimes \mathbf{A}^{q}\right) !\right| \rightarrow 0 .
$$

This is also the result of tensoring (1) with $\mathbf{A}^{p} \otimes \mathbf{A}^{q}$ and applying, in succession, the exact functors $F^{q}, !$, and $|-|$. Hence, it is exact and the required homotopy is id $\otimes K_{.}$.

The allowable resolutions of $\mathbf{N}$ and $\mathbf{N}$ ! (for elementary $\mathbf{N}$ ), are the essential ingredients in the proof of the next theorem. We shall need two others.

The first is classical adjoint associativity: Let $R$ and $S$ be rings with a morphism $R \rightarrow S$. Suppose that $A$ is a left-S, right- $R$-module; $B$ is a left-R-module; and $C$ is a left-S-module. Then $\operatorname{Hom}_{S}\left(A \otimes_{R} B, C\right) \cong \operatorname{Hom}_{S}\left(A, \operatorname{Hom}_{R}(B, C)\right)$.

The second is a lemma whose statement requires a preliminary definition. Now set $E^{h}=\sum_{i \geqslant h} e^{h} \otimes e^{i}$. We shall call an A-module $\Re$ special if: (1) $\Re \rightarrow \Pi_{h} E^{h} \Re$, $m \mapsto\left\langle E^{h} m\right\rangle$, is a $k$-isomorphism; and (2) for all $h$ and all $i \geqslant h$, (left) multiplication by $e^{h} \otimes \varphi^{h i}$ is a $k$-isomorphism $\mathfrak{N}^{h h} \rightarrow \mathfrak{N}^{h i}$. The simplest example of a special module is $\mathbf{M}$ !, where $\mathbf{M}$ is an arbitrary A-bimodule. So the full subcategory of special modules $\mathcal{S}$ contains the image of the functor !. We define a functor $r$ : $\mathcal{S} \rightarrow$ A-MOD as follows: $(r \mathfrak{K})^{i}=\mathfrak{N}^{i i}$ and, for $i \leqslant j, T^{i j}:(r \mathfrak{N})^{j} \rightarrow(r \mathfrak{N})^{i}$ is the unique $k$-morphism defined by $e^{i} \otimes \varphi^{i j} T^{i j}(x)=\varphi^{i j} \otimes e^{j} x$. It is routine to check that $T^{i j}$ is an $\mathbf{A}^{j}$-morphism and that there is an $\mathbf{A} \uparrow$-monomorphism $\varepsilon_{\mathfrak{N}}:(r \Re) ! \rightarrow \Re$. (Indeed, $r$ is the right adjoint to !: A-MOD $\rightarrow \mathcal{S}$ and $\varepsilon_{\mathfrak{I}_{\pi}}$ is the counit of the adjunction.) One can easily show that if $I_{p}$ is finite for all $p$, then $\varepsilon_{\mathscr{N}}$ is an isomorphism for all $\mathfrak{N}$ and, so, $\mathcal{S}$ is precisely the image of !. Conversely, if $I_{p}$ is infinite for some $p$, then $\Pi_{p} \Pi_{j \geqslant p} \mathbf{A}^{p} \varphi^{p j}$ is special but is not in the image of !. Clearly, $\mathcal{S}$ is closed under the formation of kernels, cokernels, and direct sums and, thus, is abelian. The product as $\mathbf{A} \uparrow$-modules of an arbitrary collection of special modules is special; the dual statement is false. (If $\mathfrak{T}$ is the coproduct as $\mathbf{A} \uparrow$-modules of a 
family $\left\{\Re_{\lambda}\right\}_{\Lambda}$ in $\S$, then $\Pi_{h} E^{h} \mathfrak{N}$ is their coproduct in $\delta$. Also, $\Re \rightarrow \Pi_{h} E^{h} \mathfrak{N}$, while always a monomorphism, is an isomorphism if and only if $\Lambda$ is finite. Note that the coproduct in $\mathcal{S}$ of $\left\{\mathbf{M}_{\lambda} !\right\}_{\Lambda}$ is $\left(\amalg_{\Lambda} \mathbf{M}_{\lambda}\right) !$.)

Our interest in special modules springs from the next lemma. Let $V$ be a $k$-bimodule and $\mathbf{M}$ an A-bimodule. Define another A-bimodule $\operatorname{Hom}_{k}(V, \mathbf{M})$ by $\operatorname{Hom}_{k}(V, \mathbf{M})^{i}=\operatorname{Hom}_{k}\left(V, \mathbf{M}^{i}\right)$. Of course, $\operatorname{Hom}_{k}(V, \mathbf{M})^{j} \rightarrow \operatorname{Hom}_{k}(V, \mathbf{M})^{i}$ is just composition with $\mathbf{M}^{j} \rightarrow \mathbf{M}^{i}$. There is an evident adjoint associativity:

$$
\operatorname{Hom}_{\mathbf{A}}(\mathbf{N} \otimes V, \mathbf{M}) \cong \operatorname{Hom}_{\mathbf{A}}\left(\mathbf{N}, \operatorname{Hom}_{k}(V, \mathbf{M})\right) \text {. }
$$

It is easy to see that $r \operatorname{Hom}_{k}(V, \mathbf{M} !)=\operatorname{Hom}_{k}(V, \mathbf{M})$ and, so, we have $\varepsilon$ : $\operatorname{Hom}_{k}(V, \mathbf{M}) ! \rightarrow \operatorname{Hom}_{k}(V, \mathbf{M} !)$. If $I_{p}$ is finite for every $p$ with $\mathbf{M}^{p} \neq 0$, then $\mathbf{M} !=$ $\Pi_{i} \Pi_{j \geq i} \mathbf{M}^{i} \varphi^{i j}$ and $\varepsilon$ is an isomorphism. In general, however, $\operatorname{Hom}_{k}(V, \mathbf{M} !)$ is not in the image of !-but it is in $\delta$, as the following lemma asserts.

Lemma. If $\mathfrak{T}$ is special, then $\operatorname{Hom}_{k}(V, \mathbf{M})$ is as well.

Proof. Let $\Re=\operatorname{Hom}_{k}(V, \mathfrak{R})$ and note that for any $i \leqslant j, \Re^{i j}=e^{i} \otimes e^{j} \Re=$ $\operatorname{Hom}_{k}\left(V, \mathfrak{T}^{i j}\right)$. It follows that $E^{h} \mathfrak{\Re}=\operatorname{Hom}_{k}\left(V, E^{h} \mathfrak{\Re}\right)$ and, hence, that $\mathfrak{R} \rightarrow$ $\Pi_{h} E^{h} \Re$ is a $k$-isomorphism. Moreover, the $k$-isomorphism $\mathfrak{N}^{h h} \rightarrow \mathfrak{N}^{h i}$ induces a $k$-isomorphism $\operatorname{Hom}_{k}\left(V, \mathfrak{T}^{h h}\right) \rightarrow \operatorname{Hom}_{k}\left(V, \mathfrak{T}^{h i}\right)$. Employing the remark above, we see that this is exactly the required isomorphism $\Re^{h h} \rightarrow \Re^{h i}$.

THEOREM. If $\mathbf{N}$ is elementary, then $\omega^{*}: \operatorname{Ext}_{\mathbf{A}}^{*}(\mathbf{N},-) \rightarrow \operatorname{Ext}_{\mathbf{A} !}^{*}(\mathbf{N} !,-!)$ is an isomorphism.

Proof. Set $\mathbf{N}=F^{q}(N)$. The theorem will follow from the opening remarks of $\S 15$ if we show that $\lambda .(N)$ induces an isomorphism of complexes $\operatorname{Hom}_{\mathbf{A} \uparrow}(\mathbf{P} .(N) !,-!) \rightarrow$ $\operatorname{Hom}_{\mathbf{A} \uparrow}\left(\mathscr{P}_{.}(N),-\right.$ !). This in turn will follow if we show that $\lambda_{i} \otimes \mathrm{id}_{n-i}$ induces an isomorphism $\operatorname{Hom}_{\mathbf{A} \uparrow}\left(\mathbf{P}_{i} ! \otimes\left|K_{n-i}\right|,-!\right) \rightarrow \operatorname{Hom}_{\mathbf{A} \uparrow}\left(\mathscr{P}_{i} \otimes\left|K_{n-1}\right|,-!\right)$. But by adjoint associativity, this is true if and only if $\lambda_{i}$ induces an isomorphism

$$
\operatorname{Hom}_{\mathbf{A} \uparrow}\left(\mathbf{P}_{i} !, \operatorname{Hom}_{k}\left(\left|K_{n-i}\right|,-!\right)\right) \rightarrow \operatorname{Hom}_{\mathbf{A} \uparrow}\left(\mathscr{P}_{i}, \operatorname{Hom}_{k}\left(\left|K_{n-i}\right|,-!\right)\right) .
$$

Since $\operatorname{Hom}_{k}\left(\left|K_{n-i}\right|, \mathbf{M} !\right)$ is special for all $\mathbf{M}$, it suffices to prove: if $\Re$ is special, then $\lambda$. induces an isomorphism of complexes

$$
\operatorname{Hom}_{\mathbf{A} \uparrow}(\mathbf{P} . !, \Re) \rightarrow \operatorname{Hom}_{\mathbf{A} \uparrow}\left(\mathscr{P}_{\text {. }}, \mathfrak{\Re}\right) \text {. }
$$

The definitions of the projectives and $\lambda$. allow us to reduce this still further to: if $\mathcal{O}$ is special, then $\lambda^{i}$ induces an isomorphism $\operatorname{Hom}_{\mathbf{A} \uparrow}(\mathbf{P}(i) !, \mathscr{T}) \rightarrow \operatorname{Hom}_{\mathbf{A} \uparrow}(\mathscr{P}(i), \mathscr{T})$. An element of the latter group is determined by the image of $e^{i} \otimes e^{q}$. Thus, $\lambda^{i}$ induces an isomorphism if and only if an element of the former group is determined by the image of $\lambda^{i}\left(e^{i} \otimes e^{q}\right)=e^{i} \otimes e^{i} \varphi^{i q}$. We demonstrate this by a simple calculation invoking the "special" nature of $\Re$.

Let $f: \mathbf{P}(i) ! \rightarrow \Re$ be an $\mathbf{A} \uparrow$-morphism. We have

$$
f\left(e^{i} \otimes e^{i} \varphi^{i q}\right)=e^{i} \otimes \varphi^{i q} f\left(e^{i} \otimes e^{i}\right),
$$

and for any $h \leqslant i, f\left(e^{h} \otimes e^{h}\right) \in \mathfrak{T}^{h h}$. But multiplication by $e^{i} \otimes \varphi^{i q}$ is an isomorphism $\mathscr{N}^{i i} \rightarrow \mathfrak{M}^{i q}$. So it is sufficient to show that $f$ is determined by $f\left(e^{i} \otimes e^{i}\right)$. Now

$$
f\left(a^{h} \otimes b^{h} \varphi^{h k}\right)=a^{h} \otimes b^{h} \varphi^{h k} f\left(e^{h} \otimes e^{h}\right) \in E^{h} \Re .
$$


Since $\mathfrak{T}=\Pi E^{h} \mathfrak{T}, \mathbf{P}(i) !=\prod E^{h} \mathbf{P}(i) !$, and $E^{h} \mathbf{P}(i) !=\amalg_{k \geqslant h} \mathbf{A}^{h e} \varphi^{h k}$, we see that $f$ is determined by the collection $\left\{f\left(e^{h} \otimes e^{h}\right) \in \mathscr{T}^{h h}\right\}_{h \leqslant i}$, subject to a coherence condition. To discover the condition, let $h \leqslant l \leqslant i$. Then

$$
e^{h} \otimes \varphi^{h l} f\left(e^{h} \otimes e^{h}\right)=f\left(e^{h} \otimes e^{h} \varphi^{h l}\right)=f\left(\varphi^{h l} \otimes e^{l} \cdot e^{l} \otimes e^{l}\right)=\varphi^{h l} \otimes e^{l} f\left(e^{l} \otimes e^{l}\right) .
$$

Thus, $\left\{f\left(e^{h} \otimes e^{h}\right)\right\}$ is a collection $\left\{m^{h} \in \mathfrak{N}^{h h}\right\}$ satisfying

$$
e^{h} \otimes \varphi^{h l} m^{h}=\varphi^{h l} \otimes e^{l} m^{l} \quad \text { whenever } h \leqslant l \leqslant i .
$$

Conversely, any collection satisfying (5) defines an element $f \in \operatorname{Hom}_{\mathbf{A} \uparrow}(\mathbf{P}(i)$ !, 이 $)$ by $f\left(e^{h} \otimes e^{h}\right)=m^{h}$. Now fix $m^{i}$ and consider (5) with $l=i$. Since multiplication by $e^{h} \otimes \varphi^{h i}$ is an isomorphism $)^{h h} \rightarrow M^{h i}, m^{i}$ determines a unique collection $\left\{m^{h}\right\}$ satisfying (5) with $l=i$. It is then an easy matter to verify that $\left\{m^{h}\right\}$ satisfies (5) for all $l$, and so $m^{i}$ determines a unique morphism $\mathbf{P}(i) ! \rightarrow \mathfrak{N}$. That is, $f$ is determined by $f\left(e^{i} \otimes e^{i}\right)$ as claimed.

The support of an $\mathbf{A}$-bimodule $\mathbf{M}$ is the set $\operatorname{supp} \mathbf{M}=\left\{i \mid M^{i} \neq 0\right\}$. If $\mathbf{N}$ is $q$-elementary-say $\mathbf{N}=F^{q}(N)$-then $\operatorname{supp} \mathbf{P}_{0}(N)=I^{q} \cup\{q\}$ and $\operatorname{supp} \mathbf{P}_{n}(N) \subseteq$ $\operatorname{supp} \mathbf{P}_{0}(N)$. So when $\operatorname{supp} \mathbf{M} \cap \operatorname{supp} \mathbf{P}_{0}(N)=\varnothing$, we find $\operatorname{Hom}_{\mathbf{A}}(\mathbf{P} .(N), \mathbf{M})=0$ and $\operatorname{Ext}_{\mathbf{A}}^{*}(\mathbf{N}, \mathbf{M})=0$.

The restricted CCT mentioned in the section title is the following

Corollary. If $\operatorname{supp} \mathbf{N}$ is finite, then $\omega^{*}: \operatorname{Ext}_{\mathbf{A}}^{*}(\mathbf{N},-) \rightarrow \operatorname{Ext}_{\mathbf{A} !}^{*}(\mathbf{N} !,-!)$ is an isomorphism.

Proof. We proceed by induction on the cardinality $n$ of $\operatorname{supp} \mathbf{N}$. (The case $n=1$ is the theorem.) Let $q$ be any minimal element in supp $\mathbf{N}$. Define $\mathbf{N}^{\prime}$ to be the $q$-elementary bimodule $\mathbf{N}^{\prime}=F^{q}\left(\mathbf{N}^{q}\right)$. Then we have an allowable short exact sequence $0 \rightarrow \mathbf{N}^{\prime} \rightarrow \mathbf{N} \rightarrow \mathbf{N}^{\prime \prime} \rightarrow 0$, and $\operatorname{supp} \mathbf{N}^{\prime \prime}$ has cardinality $n-1$. Since $\omega^{*}$ : $\operatorname{Ext}_{\mathbf{A}}^{*}(-, \mathbf{M}) \rightarrow \operatorname{Ext}_{\mathbf{A} !}^{*}(-!, \mathbf{M} !)$ is a transformation of (relative) $\delta$-functors, the fivelemma shows that it is an isomorphism at $\mathbf{N}$ if it is at $\mathbf{N}^{\prime}$ and $\mathbf{N}^{\prime \prime}$. The first of the required isomorphisms follows from the theorem; the second is the induction hypothesis.

Finally, if $\mathbf{A}$ is a finite diagram, then every module is, perforce, finitely supported. Hence, the proof of the CCT in the finite case is concluded.

Here are a few exercises which are not needed later. Fix $p$ and $q$ in $I$. If $p \$ q$, then $\operatorname{Ext}_{\mathbf{A}}^{*}\left(F^{q}(N), F^{p}(M)\right)=0$. Otherwise, let $\mathbf{A}^{\prime}$ be the restriction of $\mathbf{A}$ to $I_{p q}=\{i \mid$ $p \leqslant i \leqslant q$ \}. The restriction functor $\mathbf{A}-\mathrm{MOD} \rightarrow \mathbf{A}^{\prime}-\mathbf{M O D}, \mathbf{M} \mapsto \mathbf{M}^{\prime}$ is exact, but not full. Nonetheless, if $\operatorname{supp} \mathbf{M} \cup \operatorname{supp} \mathbf{N} \subseteq I_{p q}$, then $\operatorname{Ext}_{\mathbf{A}}^{*}(\mathbf{N}, \mathbf{M}) \cong \operatorname{Ext}_{\mathbf{A}^{\prime}}^{*}\left(\mathbf{N}^{\prime}, \mathbf{M}^{\prime}\right)$. Moreover (hard), if $I_{p q}$ is finite, linearly ordered, and has cardinality $d+1$, then $\operatorname{Ext}_{\mathbf{A}}^{*}\left(F^{q}(N), F^{p}(M)\right) \cong \operatorname{Ext}_{\mathbf{A}^{q}}^{*-d}(N, M)$.

17. The cochain map $\tau: C^{*}(\mathbf{A},-) \rightarrow C^{*}(\mathbf{A} !,-!)$. Let $\mathbf{A}$ be an arbitrary diagram and use $\psi^{*}$ to denote the composite natural transformation

$$
H^{*}(\mathbf{A},-) \stackrel{\sim}{\rightarrow} \operatorname{Ext}_{\mathbf{A}}^{*}(\mathbf{A},-) \stackrel{\omega^{*}}{\rightarrow} \operatorname{Ext}_{\mathbf{A} !}^{*}(\mathbf{A} !,-!) \stackrel{\sim}{\rightarrow} H^{*}(\mathbf{A} !,-!) .
$$

Note that $\omega^{*}$ is an isomorphism if and only if $\psi^{*}$ is. In this section we describe an explicit cochain map $\tau: C^{*}(\mathbf{A},-) \rightarrow C^{*}(\mathbf{A} !,-!)$ and show that $H^{*}(\tau)=\psi^{*}$. 
For any A-bimodule $\mathbf{M}$, a cochain $F \in C^{m}(\mathbf{A}$ !, $\mathbf{M}$ !) with $m \geqslant 1$ will be called strict if:

(i) $F\left(x_{m}, \ldots, x_{1}\right)=0$ whenever any $x_{r}$ is the unit element $e^{i}$ of some $\mathbf{A}^{i}$.

(ii) $F\left(a_{m} \varphi^{i_{m} j_{m}}, \ldots, a_{2} \varphi^{i_{2} j_{2}}, a_{1} \varphi^{i_{1} j_{1}}\right)=0$ unless $j_{m}=i_{m-1}, \ldots, j_{3}=i_{2}, j_{2}=i_{1}$.

(iii) $F\left(a_{m} \varphi^{i_{m} i_{m-1}}, \ldots, a_{2} \varphi^{i_{2} i_{1}}, a_{1} \varphi^{i_{1} i_{0}}\right) \in \mathbf{M}^{i_{m}} \varphi^{i_{m} i_{0}}$.

(We shall refer to such "matched" elements of $\mathbf{A}$ ! as strict $m$-tuples.)

For $m=0, C^{0}(\mathbf{A} !, \mathbf{M} !)=\mathbf{M}$ !; the elements of $\Pi \mathbf{M}^{i}$ will be called strict. If $F$ and $G$ are strict, then it is easy to verify that so are $\delta F, F \smile G, F{ }_{r} G$ for all $r, F{ }^{-} G$, and, when $\mathbf{M}=\mathbf{A},[F, G]$ (definitions in $\S 4$ ). An $m$-cochain will be called semistrict if (ii) and (iii) hold. When $I$ is finite, strict is equivalent to semistrict and normal: first, if $F$ is strict it is semistrict and

$$
F\left(x_{m}, \ldots, 1, \ldots, x_{1}\right)=\sum_{p \in I} F\left(x_{m}, \ldots, e^{p}, \ldots, x_{1}\right)=0,
$$

which is normality. Conversely, if $F$ is semistrict then (ii) implies

$$
F\left(x_{m}, \ldots, a^{i} \varphi^{i j}, e^{p}, a^{k} \varphi^{k l}, \ldots, x_{1}\right)=0
$$

unless $j=p=k$. But then

$$
\begin{aligned}
0 & =F\left(x_{m}, \ldots, 1, \ldots, x_{1}\right)=F\left(x_{m}, \ldots, \sum e^{p}, \ldots, x_{1}\right) \\
& =F\left(x_{m}, \ldots, a^{i} \varphi^{i j}, e^{j}, a^{j} \varphi^{j k}, \ldots, x_{1}\right) .
\end{aligned}
$$

Hence (i) holds and $F$ is strict.

Denote the subcomplex of strict cochains of $C^{*}\left(\mathbf{A}\right.$ !, $\mathbf{M}$ !) by $C_{s}^{*}(\mathbf{A}$ !, $\mathbf{M} !)$. Observe that properties (i)-(iii) are natural in $\mathbf{M}$; that is, for any $\mathbf{M} \rightarrow \mathbf{N}, C^{*}(\mathbf{A} !, \mathbf{M} !) \rightarrow$ $C^{*}\left(\mathbf{A}\right.$ !, $\mathbf{N}$ !) restricts to give $C_{s}^{*}(\mathbf{A} !, \mathbf{M} !) \rightarrow C_{s}^{*}(\mathbf{A}$ !, $\mathbf{N}$ !). The strict cohomology will be denoted $H_{s}^{*}(\mathbf{A},-!)$.

The description of the cochain map $\tau_{\mathbf{M}}: C^{*}(\mathbf{A}, \mathbf{M}) \rightarrow C^{*}(\mathbf{A} !, \mathbf{M} !)$ will be facilitated by some notation. First we define $\Delta_{q}$ to be the set of (possibly) degenerate $q$-simplices in $I: \Delta_{q}=\left\{\sigma=\left(i_{q} \leqslant \cdots \leqslant i_{0}\right)\right\}$. (If $\sigma \in \Delta_{q} \backslash \Sigma_{q}$ we set $\Gamma^{\sigma}=0$.) For $\sigma \in \Delta_{q}$ we let $\pi_{\sigma}$ be the multiplication cochain in $C^{q}\left(\mathbf{A}^{v \sigma}, \mathbf{A}^{v \sigma}\right): \pi_{\sigma}\left(a_{q}, \ldots, a_{1}\right)=$ $a_{q} \cdots a_{1}$. We shall write $\sigma \leqslant \sigma^{\prime}$ to mean $\sigma$ is a truncation of $\sigma^{\prime}$ satisfying $v \sigma=v \sigma^{\prime}$; i.e. $\sigma^{\prime}=\left(i_{q} \leqslant \cdots \leqslant i_{0}\right)$ while $\sigma=\left(i_{q} \leqslant \cdots \leqslant i_{k}\right)$. Finally, if $f \in C^{m}\left(\mathbf{A}^{p}, \mathbf{M}^{q}\right)$ and $i_{m}, \ldots, i_{1} \in I_{p}$, we interpret $f\left(a^{i_{m}}, \ldots, a^{i_{1}}\right)$ in the only reasonable fashion, namely,

$$
f\left(a^{i_{m}}, \ldots, a^{i_{1}}\right)=f\left(\varphi^{p i_{m}}\left(a^{i_{m}}\right), \ldots, \varphi^{p i_{1}}\left(a^{i_{1}}\right)\right) .
$$

Now for $\Gamma \in C^{n}(\mathbf{A}, \mathbf{M})$ we define $\tau_{\mathbf{M}}^{n} \Gamma$ by

(1) $\tau_{\mathbf{M}}^{n} \Gamma$ is strict and

$$
\tau_{\mathbf{M}}^{n} \Gamma\left(a_{n} \varphi^{i_{n} i_{n-1}}, \ldots, a_{1} \varphi^{i_{1} i_{0}}\right)=\sum_{\sigma \leqslant \sigma^{\prime}} \pi_{\sigma} \smile \Gamma^{\sigma}\left(a_{n}, \ldots, a_{1}\right) \varphi^{i_{n} i_{0}}
$$

where $\boldsymbol{\sigma}^{\prime}=\left(i_{n} \leqslant \cdots \leqslant i_{0}\right)$ and $a_{r} \in \mathbf{A}^{i_{r}}$.

This extends to $\mathbf{A}$ ! by (infinite) linearity.

The verification that $\tau_{\mathbf{M}}$ is a cochain map is a tedious computation requiring only intestinal fortitude and a few sheets of large paper. It is clear that $\tau_{\mathbf{M}}$ is natural in $\mathbf{M}$ and, so, $\tau$ induces a transformation $H^{*}(\tau): H^{*}(\mathbf{A},-) \rightarrow H^{*}(\mathbf{A} !,-!)$. Moreover, it is 
easy to see that $H^{0}(\tau)=\psi^{0}$. Now $H^{*}(\tau)$ is the unique extension of $H^{0}(\tau)$, as $H^{*}(\mathbf{A},-)$ is universal. So we have, as asserted,

THEOREM. $H^{*}(\tau)=\psi^{*}$.

Since, by definition, $\tau$ factors as $C^{*}(\mathbf{A},-) \rightarrow C_{s}^{*}(\mathbf{A} !,-!) \rightarrow C^{*}(\mathbf{A} !,-!)$, it induces a monomorphism $H^{*}(\mathbf{A},-) \rightarrow H_{s}^{*}(\mathbf{A} !,-$ !) and the inclusion induces an epimorphism $H_{s}^{*}(\mathrm{~A} !,-!) \rightarrow H^{*}(\mathrm{~A} !,-!)$. In fact, when $I$ is finite the latter is an isomorphism.

LEMMA. Suppose that I is finite. If $g \in C^{n}(\mathbf{A} !, \mathbf{M} !)$ is semistrict and $\delta g$ is strict, then there is a semistrict $h \in C^{n-1}(\mathbf{A} !, \mathbf{M} !)$ for which $g-\delta h$ is strict.

Proof. We must show that there is a semistrict $h$ for which $(g-\delta h)$ is normal. Since $\delta g$ is strict, it is normal. In $\S 7$ we gave an iterative procedure for "normalizing" $g$ when $\delta g$ is normal. A quick look at the cochains used there to adjust $g$ reveals them to be semistrict whenever $g$ is.

Proposition. When $I$ is finite $H_{s}^{*}(\mathbf{A} !,-!) \rightarrow H^{*}(\mathbf{A} !,-!)$ is an isomorphism.

Proof. We need only show $B^{*}(\mathbf{A} !, \mathbf{M} !) \cap C_{s}^{*}(\mathbf{A} !, \mathbf{M} !)=B_{s}^{*}(\mathbf{A} !, \mathbf{M} !)$. Let $f$ be a strict $n$-cochain and suppose that $f=\delta \hat{g}$. Define $g$ by

$$
g\left(x_{n-1}, \ldots, x_{1}\right)=\sum_{\Delta_{n-1}} e^{i_{n-1}} \hat{g}\left(e^{i_{n-1}} x_{n-1} e^{i_{n-2}}, e^{i_{n-2}} x_{n-2} e^{i_{n-3}}, \ldots, e^{i_{1}} x_{1} e^{i_{0}}\right) e^{i_{0}} .
$$

It is immediate that $g$ is semistrict and we claim $f=\delta g$. For this write $X=$ $\left(a_{m} \varphi^{i_{m} j_{m}}, \ldots, a_{1} \varphi^{i_{1} j_{1}}\right)$ and observe that $\delta g(X)=e^{i_{m}} \delta \hat{g}(X) e^{j_{1}}$. Hence, since $f$ is strict, $(f-\delta g)(X)=e^{i_{m}}[(f-\delta \hat{g})(X)] e^{j_{1}}=0$. Now $g$ will not be normal; however, the lemma above provides a semistrict $h$ such that $g-\delta h$ is strict. Then $f=\delta(g-\delta h)$ $\in B_{s}^{*}(\mathbf{A} !, \mathbf{M} !)$, as required.

The proposition implies that when $I$ is finite, $H^{*}(\mathbf{A},-) \rightarrow H_{s}^{*}(\mathbf{A} !,-!)$ is an isomorphism. We do not know if the same is true for arbitrary $I$; however, we can exhibit a cochain map $\hat{\tau}^{*}: C_{s}^{*}(\mathbf{A} !,-!) \rightarrow C^{*}(\mathbf{A},-)$ for which $\tau^{*}$ is a section. Before describing it, we adapt the concept of a shuffle (\$3) to our particular situation-a special case of "shuffle products with operators."

Let $\varphi=\varphi^{i j}$ and suppose that $a \in \mathbf{A}^{j}$. The strict shuffle product $\langle\varphi\rangle{ }_{{ }_{s}}\langle a\rangle$ is given by $\langle\varphi\rangle{ }_{s}\langle a\rangle=(\varphi, a)-\left(a^{\varphi}, \varphi\right)$; that is, as $\varphi$ passes $a$ it operates, so that every summand is a strict tuple. This generalizes in an inductive way to give $\left\langle\varphi^{i_{p} i_{p-1}}, \ldots, \varphi^{i_{1} i_{0}}\right\rangle *_{s}\left\langle a_{n-p}, \ldots, a_{1}\right\rangle$ (where each $a_{r} \in \mathbf{A}^{i_{0}}$ ). For convenience we shall represent the strict $p$-tuple $\left\langle\varphi^{i_{p} i_{p-1}}, \ldots, \varphi^{i_{1} i_{0}}\right\rangle$ by the corresponding $\sigma \in \Delta_{p}$, namely $\sigma=\left(i_{p} \leqslant i_{p-1} \leqslant \cdots \leqslant i_{0}\right)$.

Now let $F$ be in $C_{s}^{n}(\mathbf{A} !, \mathbf{M} !)$. Define $\hat{\tau}_{\mathbf{M}}^{n} F \in C^{n}(\mathbf{A}, \mathbf{M})$ by

$$
\left(\hat{\tau}_{\mathbf{M}}^{n} F\right)^{\sigma}\left(a_{n-p}, \ldots, a_{1}\right) \varphi^{\sigma}=F\left(\sigma *{ }_{s}\left\langle a_{n-p}, \ldots, a_{1}\right\rangle\right) .
$$

Once again, the verification that $\hat{\tau}_{\mathbf{M}}^{*}$ is a cochain map is a tedious, but elementary, computation. Also $\hat{\tau}_{\mathbf{M}}^{*}$ is manifestly natural in $\mathbf{M}$ and it is trivial to see that $\hat{\tau} \tau$ is the identity.

18. A single morphism: strict cochains. When $\omega^{*}$ is an isomorphism, $H^{*}(\mathbf{A}, \mathbf{A})$ has all the cohomological properties of a single ring as given in [G1]. In particular, it has 
a cup product which is graded commutative and a graded Lie product linked to the cup product in a particular way. On the other hand, when A consists of a single morphism $\varphi: B \rightarrow A$, as we assume for the rest of this section, then we have already given candidates for the cup product of cochains (\$5) and for a composition product (§4) whose graded commutator induces the graded Lie product. The composition is suggested by the deformation theory, since under it the square of a 2-cocycle represents the primary obstruction to extension of the 2-cocycle to a deformation. We show here that the products introduced earlier are correct. Suppose that we have a $\varphi$-module $T: N \rightarrow M$. We write $\varphi$ ! for the diagram ring and $T$ ! for the induced module.

We adopt-for this section only-the following notational convention: when $a_{1}, \ldots, a_{n} \in A$, the tuple $\left(a_{i}, a_{i+1}, \ldots, a_{j}\right)$ is denoted $a_{i}^{j}$. Similar notation applies for $B$. Also, for $F \in C^{*}(\varphi !, T !)$ we write $F^{A}$ and $F^{B}$ in place of $\left.F\right|_{A}$ and $\left.F\right|_{B}$.

Lemma. If $F \in C_{s}^{m}\left(\varphi\right.$ !, $T$ !), then there is $a G \in C_{s}^{m-1}(\varphi$ !, $T$ !) such that $\bar{F}=F+\delta G$ has $\bar{F}^{A}=F^{A}, \bar{F}^{B}=F^{B}$, and

$$
\bar{F}\left(a_{1}^{r-1}, \varphi, b_{r+1}^{m}\right)=0 \quad \text { for all } r>1 .
$$

Proof. This is purely computational. Suppose that $F$ already satisfies ( $\dagger$ ) for all $r>s$, where $2 \leqslant s \leqslant m-$ a condition vacuously satisfied for $s=m$. Define $g_{s} \in$ $C^{m-1}(\varphi$ !, $T$ !) as follows:

$$
\begin{aligned}
g_{s}\left(x_{1}, \ldots, x_{m-1}\right) & =0 \quad \text { if } x_{i} \in B \oplus A \text { for all } i \leqslant s-1 \\
g_{s}\left(x_{1}, \ldots, x_{i} \varphi, x_{i+1}, \ldots, x_{m-1}\right) & =g_{s}\left(x_{1}, \ldots, x_{i}, \varphi x_{i+1}, \ldots, x_{m-1}\right) \text { for all } i \leqslant s-2 ;
\end{aligned}
$$

and

$$
g_{s}\left(x_{1}, \ldots, x_{s-2}, a \varphi, \ldots, x_{m-1}\right)=F\left(x_{1}, \ldots, a, \varphi, \ldots, x_{m-1}\right) .
$$

Then $g_{s}$ is strict,

$$
g_{s}^{A}=0=g_{s}^{B}, \quad g_{s}\left(a_{1}^{s-2}, \varphi, b_{s+1}^{m}\right)=F\left(a_{1}^{s-2}, 1_{A}, \varphi, b_{s+1}^{m}\right)=0,
$$

and so

$$
(-1)^{s} \delta g_{s}\left(a_{1}^{s-1}, \varphi, b_{s+1}^{m}\right)=-F\left(a_{1}^{s-1}, \varphi, b_{s+1}^{m}\right) .
$$

It follows that $F+(-1)^{s} \delta g_{s}$ satisfies ( $\dagger$ ) for all $r>s-1$. The required $G$ is then $\sum_{s=2}^{m}(-1)^{s} g_{s}$.

The proof of the lemma also shows that

$$
\bar{F}\left(\varphi, b_{1}^{m-1}\right)=F\left(\varphi, b_{1}^{m-1}\right)+\sum_{s=2}^{m}(-1)^{s-1} F\left(b_{1}^{\varphi}, \ldots, b_{s-1}^{\varphi}, \varphi, b_{s}^{m-1}\right) .
$$

TheOREM. If $\Gamma \in C^{*}(\varphi, T)$ and $\Gamma^{\prime} \in C^{*}(\varphi, \varphi)$ then $\tau\left(\Gamma \bar{\circ} \Gamma^{\prime}\right)=\tau \Gamma \bar{\circ} \tau \Gamma^{\prime}+\delta G$ for some $G \in C^{*}(\varphi$ !, $T$ !).

Proof. While $\tau \Gamma$ and $\tau \Gamma^{\prime}$ satisfy $(\dagger), \tau \Gamma \bar{\sigma} \tau \Gamma^{\prime}$ need not-but it is strict. It is easy to see that $\tau\left(\Gamma^{\circ} \Gamma^{\prime}\right)^{B}=\tau \Gamma^{B} \bar{\tau} \tau \Gamma^{\prime B}=\left(\tau \Gamma^{\circ} \tau \Gamma^{\prime}\right)^{B}$ and, similarly, for $\tau\left(\Gamma_{\bar{\circ}} \Gamma^{\prime}\right)^{A}$. However, in general, $\tau\left(\Gamma^{\circ} \Gamma^{\prime}\right) \neq\left(\tau \Gamma^{\circ} \tau \Gamma^{\prime}\right)$. Let $\bar{F}$ be the cochain the lemma associates to $\tau \Gamma^{\circ} \tau \Gamma^{\prime}$. Denote the dimensions of $\Gamma$ and $\Gamma^{\prime}$ by $m$ and $n$, respectively, 
and set $a_{i}=b_{i}^{\varphi}$. Then the comment preceding the theorem shows that

$$
\bar{F}\left(\varphi, b_{1}^{m+n-2}\right)=\tau \Gamma^{\overline{0}} \tau \Gamma^{\prime}\left(\varphi, b_{1}^{m+n-2}\right)+\sum_{s=2}^{m}(-1)^{s-1} \tau \Gamma \bar{\circ} \tau \Gamma^{\prime}\left(a_{1}^{s-1}, \varphi, b_{s}^{m+n-2}\right) .
$$

We compute the right side:

$$
\begin{aligned}
\tau \Gamma \bar{\circ} \tau \Gamma^{\prime}\left(\varphi, b_{1}^{m+n-2}\right)= & \tau \Gamma\left(\tau \Gamma^{\prime}\left(\varphi, b_{1}^{n-1}\right), b_{n}^{m+n-2}\right) \\
& \quad+\sum_{i \geqslant 1}(-1)^{i(n-1)} \tau \Gamma\left(\varphi, b_{1}^{i-1}, \tau \Gamma^{\prime}\left(b_{i}^{i+n-1}\right), b_{i+n}^{m+n-2}\right) \\
= & \tau \Gamma\left(\Gamma^{A B}\left(b_{1}^{n-1}\right) \varphi, b_{n}^{m+n-2}\right)+(-1)^{n-1} \Gamma^{A B} \bar{\circ} \Gamma^{\prime B}\left(b_{1}^{m+n-2}\right) \varphi \\
= & \Gamma^{A B}\left(b_{1}^{n-1}\right) \Gamma^{A B}\left(b_{n}^{m+n-2}\right) \varphi+\tau \Gamma\left(\Gamma^{A B}\left(b_{1}^{n-1}\right), a_{n}^{m+n-2}\right) \varphi \\
& +(-1)^{n-1} \Gamma^{A B} \bar{\circ} \Gamma^{\prime B}\left(b_{1}^{m+n-2}\right) \varphi \\
= & {\left[\Gamma^{A B} \smile \Gamma^{A B}+\Gamma^{A} \bar{o}_{1} \Gamma^{A B}+(-1)^{n-1} \Gamma^{A B} \bar{\circ} \Gamma^{\prime B}\right]\left(b_{1}^{m+n-2}\right) \varphi . }
\end{aligned}
$$

Next,

$$
\begin{aligned}
\tau \Gamma \overline{\mathrm{o}} \tau \Gamma^{\prime}\left(a_{1}^{s-1}, \varphi, b_{s}^{m+n-2}\right) & =(-1)^{(s-1)(n-1)} \tau \Gamma \overline{\mathrm{o}}_{s} \tau \Gamma^{\prime}\left(a_{1}^{s-1}, \varphi, b_{s}^{m+n-2}\right) \\
& =(-1)^{(s-1)(n-1)} \tau \Gamma\left(a_{1}^{s-1}, \tau \Gamma^{\prime}\left(\varphi, b_{s}^{s+n-2}\right), b_{s+n-1}^{m+n-2}\right) \\
& =(-1)^{(s-1)(n-1)} \Gamma^{A}\left(a_{1}^{s-1}, \Gamma^{A B}\left(b_{s}^{s+n-2}\right), a_{s+n-1}^{m+n-2}\right) \varphi \\
& =(-1)^{(s-1)(n-1)} \Gamma^{A} \overline{\mathrm{o}}_{s} \Gamma^{\prime A B}\left(b_{1}^{m+n-2}\right) \varphi .
\end{aligned}
$$

Combining these two calculations (and noting that the dimension of $\Gamma^{\prime A B}$ is $n-1$ ), one sees that

$$
\bar{F}\left(\varphi, b_{1}^{m+n-2}\right)=\left\{\Gamma^{A} \bar{\circ} \Gamma^{A B}+(-1)^{n-1} \Gamma^{A B} \bar{\circ} \Gamma^{\prime B}+\Gamma^{A B} \smile \Gamma^{A B}\right\}\left(b_{1}^{m+n-2}\right) \varphi .
$$

But this is precisely the definition of $\tau\left(\Gamma \bar{\circ} \Gamma^{\prime}\right)\left(\varphi, b_{1}^{m+n-2}\right)$. Hence, $\bar{F}=\tau\left(\Gamma \bar{\circ} \Gamma^{\prime}\right)$.

Notice that if $\Gamma^{A}=0$, then $\tau\left(\Gamma \bar{\circ} \Gamma^{\prime}\right)=\tau \Gamma \bar{\circ} \tau \Gamma^{\prime}$. From the lemma, it follows that if $\Gamma \in Z^{m}(\varphi, \varphi)$ and $\Gamma^{\prime} \in Z^{n}(\varphi, \varphi)$, then the graded commutator $\left[\Gamma, \Gamma^{\prime}\right]=\Gamma \bar{\circ} \Gamma^{\prime}$ $-(-1)^{(m-1)(n-1)} \Gamma^{\prime} \bar{\circ} \Gamma$ is in $Z^{m+n-1}(\varphi, \varphi)$, and this indeed induces a product on $H^{*}(\varphi, \varphi)$ which agrees with the usual graded Lie product on $H^{*}(\varphi !, \varphi !)$ through $H^{*}(\tau)$. In particular, if $\Gamma^{\prime \prime} \in Z^{r}(\varphi, \varphi)$, then

$$
(-1)^{m r}\left[\Gamma,\left[\Gamma^{\prime}, \Gamma^{\prime \prime}\right]\right]+(-1)^{n m}\left[\Gamma^{\prime},\left[\Gamma^{\prime \prime}, \Gamma\right]\right]+(-1)^{r n}\left[\Gamma^{\prime \prime},\left[\Gamma, \Gamma^{\prime}\right]\right]
$$

is a coboundary.

With the same notations as before, recall that in $\$ 5$ we defined $\Gamma^{\prime} \quad \Gamma$ by

$$
\Gamma^{\prime} \smile \Gamma=\left(\Gamma^{B} \smile \Gamma^{B}, \Gamma^{A} \smile \Gamma^{A} ; \Gamma^{\prime A B} \smile \varphi \Gamma^{B}+(-1)^{n} \Gamma^{A} \varphi \smile \Gamma^{A B}\right) .
$$

A computation similar to the previous one, but simpler, shows that $\tau\left(\Gamma^{\prime}-\Gamma\right)$ and $\tau \Gamma^{\prime} \smile \tau \Gamma$ are cohomologous. As before, if $\Gamma^{A}=0$, then $\tau\left(\Gamma-\Gamma^{\prime}\right)=\tau \Gamma-\tau \Gamma^{\prime}$. We have, therefore, an explicit realization in terms of cochains of the cohomology operations on $H^{*}(\varphi, \varphi)$. 
19. The Nijenhuis-Richardson theory. In [NR] Nijenhuis and Richardson consider the problem of deforming a monomorphism $\varphi: B \rightarrow A$, the structures of $B$ and $A$ remaining unchanged. They show the infinitesimals to be elements of $H^{1}(B, A)$, and the primary obstruction to integrating $\gamma \in H^{1}$ to be $\gamma \smile \gamma \in H^{2}$. (Intuitively, one wants "small" motions of $B$ inside $A$, but those obtained by "small" inner automorphisms of $A$ are considered trivial.) In a later paper, Richardson [Rch] addresses the problem of deforming a subalgebra of a fixed algebra, i.e., of deforming a monomorphism $\varphi: B \rightarrow A$ where the multiplication in $A$ remains unchanged. Here, one wants submodules of $A$ which are "near" $B$ and which remain closed under the multiplication in $A$; two such which differ by a "small" inner automorphism of $A$ are considered equivalent. More precisely, one wants $k[[t]]$-submodules $B_{t}$ of $A[[t]]$ which are closed under the multiplication in $A[[t]]$ and which reduce, modulo $t$, to $B$; one considers $B_{t} \sim B_{t}^{\prime}$ if there is an element $a_{t}=1+t a_{1}+t^{2} a_{2}+\cdots \in A[[t]]$ such that $B_{t}^{\prime}=a_{t} B_{t} a_{t}^{-1}$. In this case, the infinitesimals are elements of $H^{1}(B, A / B)$ and the obstructions lie in $H^{2}(B, A / B)$. [As usual, the $B$-module structure on $A$ is defined through $\varphi$. The submodule $\varphi(B)$ is identified with $B$.] Since Nijenhuis and Richardson require the base ring to be a field, $\varphi: B \rightarrow A$ is automatically allowable and induces an exact triangle:

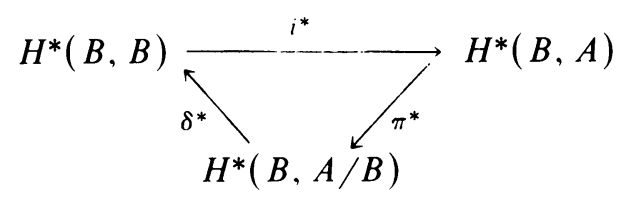

(Note that $\delta^{*}$ raises dimension by 1.) It is immediate from the definitions that $i^{*}$ is a cup product homomorphism. In [N] Nijenhuis shows that $H^{*}(B, A / B)$ carries a graded Lie algebra structure, which he calls a "cup" structure, since the grading is by dimension (in contrast to the situation in $\S 4$ ). Moreover, he shows $\delta^{*}$ is a graded Lie homomorphism, $\pi^{*}$ is a homomorphism of "cup" structures, and the image of $i^{*}$ lies in the center of $H^{*}(B, A)$, relative to the cup product. [Here "center" must be interpreted in the sense of graded algebras as elaborated below.]

We reexamine and extend these results. Naturally, if we are to have the exact triangle above, we must assume, for the first time, that $\varphi$ is allowable. We shall also assume that $\varphi$ is a monomorphism and identify $\varphi(B)$ with $B$. A deformation of $\varphi$ in which $A$ remains fixed is equivalent to one whose infinitesimal $\Gamma=\left(\Gamma^{B}, \Gamma^{A} ; \Gamma^{A B}\right)$ $\in C^{2}(\varphi, \varphi)$ has $\Gamma^{A}=0$. We define the subcomplex $C_{0}^{*}(\varphi, \varphi)$ of $C^{*}(\varphi, \varphi)$ to consist of those $\Gamma$ having $\Gamma^{A}=0$. Observe that $C_{0}^{*}(\varphi, \varphi)$ is isomorphic to the mapping cylinder of the obvious cochain map $C^{*}(B, B) \rightarrow C^{*}(B, A)$. Now consider the complex $C^{*}(B, A / B)$ with its coboundary operator adjusted by a factor of -1 . (This will not affect the cohomology.) There is a natural cochain map $C_{0}^{*}(\varphi, \varphi) \rightarrow$ $C^{*-1}(B, A / B)\left(\Gamma^{B}, 0 ; \Gamma^{A B}\right) \rightarrow \pi \Gamma^{A B}$, where $\pi: A \rightarrow A / B$ is the projection. As $\varphi$ is allowable, this is an epimorphism. One easily sees that its kernel is the mapping cylinder of id: $C^{*}(B, B) \rightarrow C^{*}(B, B)$ and this cylinder has trivial cohomology. Thus, $H_{0}^{*}(\varphi, \varphi) \rightarrow H^{*-1}(B, A / B)$ is an isomorphism. The inverse sends the class of $f \in C^{m-1}(B, A / B)$ to the class of $(\delta \hat{f}, 0 ; \hat{f}) \in C_{0}^{m}(\varphi, \varphi)$, where $\pi \hat{f}=f$. The formulae for the cup and composition products in $C^{*}(\varphi, \varphi)$ show that $C_{0}^{*}(\varphi, \varphi)$ is 
closed-in fact, is an ideal-under both multiplications. Now the cochain monomorphism $\tau: C^{*}(\varphi, \varphi) \rightarrow C_{s}^{*}(\varphi !, \varphi$ !) becomes a homomorphism for both products when restricted to $C_{0}^{*}(\varphi, \varphi)$ [cf. §18]. Also, if $f^{m}, g^{n} \in C^{*}(\varphi !, \varphi !)$ then $\delta\left[f^{m}, g^{n}\right]=$ $(-1)^{n-1}\left[\delta f^{m}, g^{n}\right]+\left[f^{m}, \delta g^{n}\right]$ and $\delta\left(f^{m} \smile g^{n}\right)=\delta f^{m} \smile g^{n}+(-1)^{m} f^{m} \smile \delta g^{n}$. It follows that $\left[Z_{0}^{*}, Z_{0}^{*}\right] \subseteq Z_{0}^{*},\left[Z_{0}^{*}, B_{0}^{*}\right] \subseteq B_{0}^{*}, Z_{0}^{*} \smile Z_{0}^{*} \subseteq Z_{0}^{*}$, and $Z_{0}^{*} \smile B_{0}^{*} \subseteq B_{0}^{*}$. Therefore, $H_{0}^{*}(\varphi, \varphi)$ inherits from $C_{0}^{*}(\varphi, \varphi)$ both a cup and a graded Lie product. These satisfy all the formal identities which hold in $C^{*}(\varphi !, \varphi !)$. Moreover, $H_{0}^{*}(\varphi, \varphi) \rightarrow$ $H^{*}(\varphi, \varphi) \cong H^{*}(\varphi !, \varphi !)$ is a morphism for both the cup and graded Lie structures.

To express the Lie and cup products in $H^{*}(B, A / B)$ in terms of cocycles, let $f \in Z^{m}(B, A / B), g \in Z^{n}(B, A / B)$ and let $\hat{f}, \hat{g}$ be liftings to elements of $C^{*}(B, A)$. Then, in $C_{0}^{*}(\varphi, \varphi)$, one has

$$
(\delta \hat{f}, 0 ; \hat{f})^{\bar{\circ}}(\delta \hat{g}, 0 ; \hat{g})=\left(\delta \hat{f}^{\bar{\circ}} \delta \hat{g}, 0 ;(-1)^{n} \hat{f}^{\bar{\circ}} \delta \hat{g}+\hat{g} \smile \hat{f}\right) .
$$

Denote $\pi\left((-1)^{n} \hat{f}^{\bar{\circ}} \delta \hat{g}+\hat{g} \smile \hat{f}\right)$ by $f^{\bar{\circ}} g$ (although this depends on the liftings), and set $[f, g]=f^{\bar{\circ}} g-(-1)^{m n} g \bar{\circ} f$. Our theory shows that this is a cocycle whose class is independent of the choice of liftings and is the desired Lie product of the classes of $f$ and $g$. Nijenhuis calls this the "cup" product, but the true cup of the classes of $f$ and $g$ is the class of $\pi(\hat{f} \smile \delta \hat{g})$, as one sees from the formula $(\delta \hat{f}, 0 ; \hat{f}) \smile(\delta \hat{g}, 0 ; \hat{g})=$ $(\delta \hat{f} \smile \delta \hat{g}, 0 ; \hat{f} \smile \delta \hat{g})$. We see that the Lie and cup products on $H^{*}(B, A / B)$ enjoy all the properties exhibited in [N] if one counts the degrees correctly. The elements of $H^{m}(B, A / B)$ must be viewed as having degree $m$ for the Lie product and degree $m+1$ for the cup product because the isomorphism $H^{*}(B, A / B) \rightarrow H_{0}^{*+1}(\varphi, \varphi)$ raises dimension by 1 . Moreover, $\delta^{*}: H^{*}(B, A / B) \rightarrow H^{*+1}(B, B)$ preserves both cup and Lie products and the relations between them since $\delta^{*}=H^{*}(B, A / B) \rightarrow$ $H_{0}^{*+1}(\varphi, \varphi) \rightarrow H^{*+1}(B, B)$. (The latter morphism is induced by $\left(\Gamma^{B}, 0 ; \Gamma^{A B}\right) \rightarrow \Gamma^{B}$ and trivially preserves both products.)

Now consider deformations of $\varphi$ in which both $B$ and $A$ remain fixed. Such a deformation is equivalent to one whose infinitesimal $\left(\Gamma^{B}, \Gamma^{A} ; \Gamma^{A B}\right)$ has $\Gamma^{B}=0=$ $\Gamma^{A}$. Define the subcomplex $C_{0,0}^{*}(\varphi, \varphi)$ of $C_{0}^{*}(\varphi, \varphi)$ to consist of those cochains having $\Gamma^{B}=0=\Gamma^{A}$. Observe that $C_{0,0}^{*}(\varphi, \varphi)$ is isomorphic to $C^{*-1}(B, A)$, with its coboundary operator adjusted by a factor of -1 . Clearly, $C_{0,0}^{*}(\varphi, \varphi)$ is also closed under both the cup and composition products and all the preceding arguments apply. However, if $\Gamma=\left(0,0 ; \Gamma^{A B}\right)$ and $\Delta=\left(0,0 ; \Delta^{A B}\right)$, then $\Gamma \bar{\circ}=$ $\left(0,0 ; \Delta^{A B} \smile \Gamma^{A B}\right)$, while $\Gamma \smile \Delta$ vanishes. The morphism $\pi^{*}: H^{*}(B, A) \rightarrow H^{*}(B, A / B)$, therefore, indeed carries the graded Lie structure obtained by taking graded commutators of the cup product in $H^{*}(B, A)$ to the graded Lie structure obtained from composition in $H^{*}(B, A / B)$ (Nijenhuis' "cup"). This confusion arises because the cohomology groups are intrinsic but the complexes from which they are computed are not, and some serve poorly for deformation theory. Using $C^{*}(\varphi, \varphi)$ seems natural and clarifies the results.

We return to Nijenhuis' observation that $i^{*}$ carries $H^{*}(B, B)$ into the "center" of $H^{*}(B, A)$. A graded algebra $\mathfrak{A}=\amalg \mathfrak{A}^{m}$ is called graded commutative (or sometimes just "commutative") if $a^{m} b^{n}=(-1)^{m n} b^{n} a^{m}$ for $a^{m} \in \mathfrak{A}^{m}, b^{n} \in \mathfrak{U}^{n}$. For any $\mathfrak{A}$, the set of $a^{m}$ which have this property for all $b^{n}$ is closed under multiplication, and its 
linear span is a subalgebra called the center. The cup product in $H^{*}(B, B)$ is graded commutative, as shown in [G1], for if $f^{m} \in Z^{m}(B, B), g^{n} \in Z^{n}(B, B)$, then

$$
[f, g]^{\smile}=f^{m}-g^{n}-(-1)^{m n} g^{n} \smile f^{m}=-(-1)^{(m+1) n} \cdot \delta\left(f^{\bar{\circ}} g\right) \text {. }
$$

If $B$ is a subalgebra of $A$, then this will continue to be meaningful for $f \in Z^{m}(B, A)$ and $g \in Z^{n}(B, B)$, since we can then still form $f^{\bar{\circ}} g$; this is Nijenhuis' observation. (Nijenhuis also makes the important observation that for characteristic 2 one must adopt a stronger definition of "graded Lie algebra.")

When $B=A$ and $\varphi$ is the identity, $A / B=0$. The foregoing shows that if $f^{m} \in C^{m}(A, A), g^{n} \in C^{n}(A, A)$, then the pairing defined by $f^{m} * g^{n}=(-1)^{n} f \circ \delta g$ $+g \smile f \in C^{m+n}(A, A)$ is a pre-Lie product. When $g$ is a cocycle $f * g=g \smile f$. Hence the commutator of the * product induces the trivial multiplication on the cohomology.

Finally, we reexamine the general deformation of a monomorphism $\varphi: B \rightarrow A$, which we view as in inclusion, by using a complex analogous to Nijenhuis'. There is a cochain map $f: C^{*}(A, A) \rightarrow C^{*}(B, A / B)$ which first restricts the arguments to $B$ and then reduces the values modulo $B$. The corresponding mapping cylinder complex has $Z^{*}=C^{*}(A, A) \oplus C^{*-1}(B, A / B)$ and $\delta(x, y)=(\delta x, \pi x \varphi-\delta y)$ where $x \varphi \in C^{*}(B, A)$ is the restriction of $x$. There is a natural cochain map $\chi: C^{*}(\varphi, \varphi) \rightarrow$ $Z^{*},\left(\Gamma^{B}, \Gamma^{A} ; \Gamma^{A B}\right) \mapsto\left(\Gamma^{A}, \pi \Gamma^{A B}\right)$. Since $\varphi$ is allowable, $\chi$ is an epimorphism. It is easy to see that $\operatorname{ker} \chi$ is the mapping cylinder of id: $C^{*}(B, B) \rightarrow C^{*}(B, B)$, which has trivial cohomology. Thus, $H(\chi): H^{*}(\varphi, \varphi) \rightarrow H^{*}(Z)$ is an isomorphism. The elements of $H^{2}(Z)$ may be identified with the infinitesimal deformations of $\varphi$. Also, $H^{*}(Z)$ then carries graded Lie and graded commutative associative algebra structures, related as in [G1].

20. Idempotents and deformations. In this section $A$ will be a $k$-algebra and $A_{t}$ will be a deformation of $A$. The product of $x$ and $y$ in both $A$ and the trivial deformation of $A$ will be denoted $x y$; in $A_{t}$ it will be denoted $x \cdot y$. (Recall: $A_{t}=A[[t]]$ as a module over $k_{t}=k[[t]]$.)

Lemma. If $e \in A$ is idempotent then there is a unique idempotent $e_{t}=\sum e_{i} t^{i} \in A_{t}$ such that $e_{0}=e$ and $e_{t} e=e e_{t}$.

Proof. Suppose we have found $e_{0}, \ldots, e_{n}(n \geqslant 0)$. Then $e_{t} \cdot e_{t}=e_{t}$ implies that $e_{n+1} e+e e_{n+1}+a=e_{n+1}$, where $a$ is uniquely determined by $e_{0}, \ldots, e_{n}$. So we must solve $x-e x-x e=a$ and $e x=x e$ simultaneously. These combine to give $x-2 e x$ $=a$. (Note also that $e a=a e=-e x e$.) If $A$ has a unit then $(1-2 e)^{2}=1$ and the unique solution is $x=(1-2 e) a=a-2 e a$. Otherwise, observe that $a-2 e a$ is nonetheless a solution. It will be unique if and only if $x-2 e x=0$ has no nontrivial solutions. But a solution of the latter satisfies $e x=2 e x$. Hence, $e x=0$ and $x=2 e x=0$, yielding the required uniqueness.

The lemma, in effect, begins with an "approximate" idempotent $e$ and refines it to a true idempotent $e_{t}$. Another way to do this: observe that $f(x)=-x^{2}(1-2 x)$. $\left(1+4 x-4 x^{2}\right)$ has constant term zero, while $f(x)-x \in\left(x^{2}-x\right) \mathbf{Z}[x]$ and $f(x)^{2}$ $-f(x) \in\left(x^{2}-x\right)^{2} \mathbf{Z}[x]$. Consequently, given any ring $\Lambda$ (unital or not), two-sided 
ideal $\mathfrak{A}$, and $e \in \Lambda$ with $e^{2}-e \in \mathfrak{A}$, we find $f(e) \in \Lambda, f(e)-e \in \mathfrak{A}$ and $f(e)^{2}-$ $f(e) \in \mathfrak{A}^{2}$. It follows that $\left\{f^{n}(e)\right\}$ is a Cauchy sequence in the $\mathfrak{A}$-adic topology, and if $\Lambda$ is complete it converges to an idempotent $\bar{e} \in \Lambda$ with $\bar{e}-e \in \mathfrak{A}$. [The polynomial $f(x)$ is obtained by formally applying Newton's method to solve $x^{2}-x=0$. If $x_{0}=e$ then $x_{1}=-e^{2}(1-2 e)^{-1}$ and $x_{1}^{2}-x_{1}=\left(e^{2}-e\right)(1-2 e)^{-2}$. Since $(1-2 e)\left(1+4 e-4 e^{2}\right)$ is an inverse modulo $\mathfrak{U}^{2}$ for $1-2 e$, we take $x_{1}=$ $-e^{2}(1-2 e)\left(1+4 e-4 e^{2}\right)$ and so obtain $f(x)$.]

Lemma. If $e \in A$ is idempotent then there is an idempotent $e_{t}=\sum e_{i} t^{i} \in A_{t}$ such that $e_{0}=e$ and $e \cdot e_{t}=e_{t} \cdot e$.

Proof. Apply the comments above to $\Lambda=A_{t}, \mathfrak{U}=(t)$. Since $A_{t}$ is $t$-adically complete, $\left\{f^{n}(e)\right\}$ converges to $e_{t}$. Each $e_{i}$ is obtained by evaluating an element of $A_{t}[x]$ at $e$ and, hence, commutes with $e$.

We pause to repair a lacuna in [G4]. There the idea was to find a polynomial which has the features of $f(x)$ by searching for the simplest $g(x) \in \mathbf{Z}[x]$ satisfying $x^{2}(x-1)^{2} \mid g(x)^{2}-g(x)$. It is $g(x)=2 x^{3}-3 x^{2}+1$. If $\Lambda$ has a unit then when $e^{2}-e \in \mathfrak{A}$ we have $g(e)^{2}-g(e) \in \mathfrak{A}^{2}$. In [G4] it was, in effect, assumed that $g(e)-e \in \mathfrak{A}$. Actually, $g(e)-(1-e) \in \mathfrak{A}$. This difficulty may be remedied by taking, instead, $g(g(x))=g^{2}(x)$. Then $g^{2}(e)^{2}-g^{2}(e) \in \mathfrak{U}^{4}$ and $g^{2}(e)-e \in \mathfrak{A}$. So we may use $g^{2}(x)$ for the same purpose as $f(x)$ above. [Note: $g^{2}(x)$ has constant term zero.]

LEMMA. If $e_{t}=\sum e_{i} t^{i} \in A_{t}$ is an idempotent with $e_{0}$ central in $A$, then $e_{t}$ is central in $A_{t}$. Moreover, if $e \in A$ is a central idempotent then there is a unique idempotent $e_{t}=\sum e_{i} t^{i} \in A_{t}$ with $e_{0}=e$.

Proof. Let $\Lambda$ be any ring. For any $\lambda \in \Lambda$, denote the derivation $x \mapsto \lambda x-x \lambda$ by ad $\lambda$. Then

$$
(\operatorname{ad} \lambda)^{n} x=\sum_{i=0}^{n}(-1)^{i}\left(\begin{array}{c}
n \\
i
\end{array}\right) \lambda^{n-i} x \lambda^{i} .
$$

So, if $f$ is an idempotent and $n$ is odd, we have $(\operatorname{ad} f)^{n} x=\left(\operatorname{ad} f^{n}\right) x=(\operatorname{ad} f) x$. Now suppose that $\Lambda$ contains a two-sided ideal $\mathfrak{A}$ satisfying: for all $n \geqslant 0, x \in \mathfrak{A}^{n}$ implies $(\operatorname{ad} f) x \in \mathfrak{U}^{n+1}$. Then, in fact, $(\operatorname{ad} f) x \in \cap \mathfrak{A}^{n}$ and, if $\cap \mathfrak{A}^{n}=0$, we see that $f$ is central in $\Lambda$. Applying this with $\Lambda=A_{t}, \mathfrak{A}=(t)$, and $f=e_{t}$ yields the first claim. (The centrality of $e_{0}$ gives $\left(\operatorname{ad} e_{t}\right) x \in(t)$ for all $x$.)

For the second claim, suppose $e_{t}=\sum e_{i} t^{i}$ and $\bar{e}_{t}=\sum \bar{e}_{i} t^{i}$ are idempotents with $e_{0}=e=\bar{e}_{0}$. (The existence of at least one such is guaranteed by each of the above lemmas.) Then $e-e^{2}=0$ implies that $e_{t}-e_{t} \cdot \bar{e}_{t} \in(t)$. But $e_{t}$ and $\bar{e}_{t}$ are central, so $e_{t}-e_{t} \cdot \bar{e}_{t}$ is an idempotent and must then lie in $\cap\left(t^{n}\right)$. That is, $e_{t}-e_{t} \bar{e}_{t}=0$ and $e_{t}=e_{t} \cdot \bar{e}_{t}$. Symmetrically, $e_{t} \cdot \bar{e}_{t}=\bar{e}_{t}$ and we have $e_{t}=\bar{e}_{t}$ as required.

Suppose that $A$ is unital. Taking $e=1$ we obtain a unique (central) idempotent $e_{t} \in A_{t}$. Now, $x \mapsto e_{t} \cdot x$ is a $k_{t}$-module automorphism of $A_{t}$ since it is the identity modulo $(t)$. Hence, for any $a_{t} \in A_{t}, e_{t} \cdot x=a_{t}$ has a unique solution. The equation $a_{t}=e_{t} \cdot x=e_{t} \cdot e_{t} \cdot x=e_{t} \cdot a_{t}$ shows that $x=a_{t}$ and that $A_{t}$ is unital with $e_{t}$ as unit. 
Thus, a deformation of a unital ring is again unital. Indeed, we may assume that 1 remains the unit. For, if we define a $k_{t}$-automorphism $\varepsilon_{t}$ of $A_{t}$ by $\varepsilon_{t}(x)=e_{t} x$, then $\mu_{t}$ defined by $\varepsilon_{t} \mu_{t}(x, y)=\varepsilon_{t} x \cdot \varepsilon_{t} y$ is an equivalent deformation which has 1 for its unit.

Observe, moreover, that any deformation $\varphi_{t}$ of a unital morphism $\varphi: B \rightarrow A$ is necessarily unital. For if $e_{t}$ is the unit of $B_{t}$ then $\varphi_{t}\left(e_{t}\right)$ is an idempotent of $A_{t}$ which reduces to $\mathrm{l}_{A}$ modulo $(t)$; hence, it is the unit of $A_{t}$. It follows that in any deformation of a diagram the rings and morphisms are all unital. Hence if $\mathbf{A}_{t}$ is a deformation of $\mathbf{A}$ then $\# \mathbf{A}_{t}$ is a deformation of $\# \mathbf{A}$. Moreover, any equivalence of deformations of $\mathbf{A}$ extends to an equivalence of deformations of \# $\mathbf{A}$. Combining these comments with those at the end of $\$ 2$ we find: the deformation theories of $\mathbf{A}$ and \# A are naturally equivalent. The purpose of the next section is to compare the deformation theories of $\mathbf{A}$ and (\# $\mathbf{A})$ !.

As in the case of a ring, any deformation $\mathbf{A}_{t}$ of a diagram $\mathbf{A}$ is equivalent to one-say $\overline{\mathbf{A}}_{t}$-in which $e^{i}$ remains the unit of $\overline{\mathbf{A}}_{t}^{i}$. For if $e_{t}^{i}$ denotes the unit of $\mathbf{A}_{t}^{i}$ we define a $k_{t}$-module automorphism $\varepsilon_{t}^{i}$ of $\mathbf{A}_{t}^{i}$ by $\varepsilon_{t}^{i}\left(a^{i}\right)=e_{t}^{i} a^{i}$. Then $\overline{\mathbf{A}}_{t}$ is given by $\varepsilon_{t}^{i} \bar{\alpha}_{t}^{i}\left(a^{i}, b^{i}\right)=\alpha_{t}\left(\varepsilon_{t}^{i} a^{i}, \varepsilon_{t}^{i} b^{i}\right)$ and $\varepsilon_{t}^{i} \bar{\varphi}_{t}^{i j}=\varphi_{t}^{i j} \varepsilon_{t}^{j}$.

If $e$ and $f$ are idempotents in a ring with $e f=0=f e$, we shall say they are orthogonal and write $e \perp f$.

Proposition. A deformation of a finite product of unital algebras is equivalent to a product of deformations of the individual algebras.

Proof. By induction it suffices to consider a product of two algebras. Let $A=A^{1} \times A^{2}$ with $e^{i}$ being the unit of $A^{i}$ and $1=e^{1}+e^{2}$ (so $A^{i}=e^{i} A$ and $e^{i}$ is central in $A$ ). Then there are unique central idempotents $e_{t}^{i} \in A_{t}$ with $e_{0}^{i}=e^{i}$. Observe that $e_{t}^{1} \cdot e_{t}^{2}=e_{t}^{2} \cdot e_{t}^{1}$ is in the ideal $(t)$ and, by centrality, is an idempotent. Hence, $e_{t}^{1} \cdot e_{t}^{2} \in \bigcap\left(t^{n}\right)$ and we have $e_{t}^{1} \perp e_{t}^{2}$. (It follows that $e_{t}^{1}+e_{t}^{2}$ is an idempotent and, since its constant term is 1 , it is the unit of $\left.A_{t}.\right)$ Next, $g_{t}(x)=e_{t}^{1} \cdot e^{1} x+$ $e_{t}^{2} \cdot e^{2} x$ is a $k_{t}$-module automorphism of $A_{t}$ since it is the identity modulo $(t)$. Note that $g_{t}\left(A^{i}[[t]]\right)=e_{t}^{i} \cdot A_{t}$. We define an equivalent deformation $\mu_{t}$ by $g_{t} \mu_{t}(x, y)=$ $g_{t} x \cdot g_{t} y$. It is immediate that $\mu_{t}$ is a product of deformations of $A^{1}$ and $A^{2}$.

In the last proof neither $e_{t}^{1}$ nor $e_{t}^{2}$ need be idempotent under $\mu_{t}$. However, there will be unique idempotents $\bar{e}_{t}^{i} \in A_{t}^{1} \times A_{t}^{2}$ which necessarily will be the units of $A_{t}^{1}$ and $A_{t}^{2}$. If we now replace $A_{t}^{1}$ and $A_{t}^{2}$ by equivalent deformations we may assume, as earlier, that $e^{1}$ and $e^{2}$ are their units and, so, $1=e^{1}+e^{2}$ is the unit of $A_{t}^{1} \times A_{t}^{2}$.

Now let $I$ be a poset and suppose that $A$ has idempotents satisfying: if $i \$ j$ then $e^{i} a e^{j}=0$ for all $a \in A$. (Example: $A=\mathbf{A}$ ! for any diagram A over I.) Let $\left\{e_{t}^{i}\right\}_{I}$ be a set of idempotents in $A_{t}$ which have $e_{0}^{i}=e^{i}$. Then when $i \$ j$ we have $e_{t}^{i} \cdot a_{t} \cdot e_{t}^{j} \in(t)$ for all $a_{t} \in A_{t}$. But then $e_{t}^{i} \cdot a_{t} \cdot e_{t}^{j}=e_{t}^{i} \cdot e_{t}^{i} \cdot a_{t} \cdot e_{t}^{j} \cdot e_{t}^{j}$ is the ideal $\left(t^{2}\right)$. Iterating, we see: if $i \$ j$, then $e_{t}^{i} \cdot a_{t} \cdot e_{t}^{j}=0$ for all $a_{t} \in A_{t}$. In particular, when $i \neq j$ we have $e_{t}^{i} \cdot e_{t}^{j}=0$.

We now impose the condition on $I$ that $I_{p}$ be finite for all $p \in I$. Suppose that there are orthogonal idempotents $\left\{e^{i}\right\}_{I}$ in $A$ such that when $i \$ j, e^{i} a e^{j}=0$ for all $a \in A$. We shall consider collections of idempotents $\left\{e_{t}^{i}\right\}_{I}$ in $A_{t}$ with $e_{0}^{i}=e^{i}$. Note 
that for any such collection we have $e_{t}^{i} \cdot a_{t} \cdot e_{t}^{j}=0$ for all $a_{t} \in A_{t}$ when $i \$ j$. Let $\mathscr{F}$ be a filter in $I$ maximal with respect to the property:

(*) for some collection $\left\{e_{t}^{i}\right\}_{I}$, we have $e_{t}^{j} \perp e_{t}^{k}$ if $j, k \in \mathscr{F}$ and $j \neq k$.

Let $p$ be a maximal element in $I \backslash \mathcal{F}$. Set $\overline{e_{t}^{p}}=e_{t}^{p}-\sum_{i>p} e_{t}^{p} \cdot e_{t}^{i}$ and $\overline{e_{t}^{j}}=e_{t}^{j}$ for $j \neq p$. Then since $e_{t}^{i} \cdot e_{t}^{j}=0$ when $i \neq j$, it is immediate that $e_{t}^{i} \perp \overline{e_{t}^{p}}$ when $i \in \mathcal{F}$ and, so, (*) is satisfied for the collection $\left\{e_{t}^{i}\right\}_{I}$ and the larger filter $\mathscr{F} \cup\{p\}$. Hence, we may orthogonalize the idempotents in $A_{t}$. That is, there is a collection of orthogonal idempotents $\left\{e_{t}^{i}\right\}$ in $A_{t}$ satisfying $e_{0}^{i}=e^{i}$ and, when $i \$ j, e_{t}^{i} \cdot a_{t} \cdot e_{t}^{j}=0$ for all $a_{t} \in A_{t}$.

Now suppose that $A=\mathbf{A}$ ! for a diagram over a finite poset $I$ and let $\left\{e_{t}^{i}\right\}_{I}$ be the idempotents obtained above. Observe that since the constant term of $\Sigma e_{t}^{i}$ is $\Sigma e^{i}=1$, the unit for $A_{t}$ is $\sum e_{t}^{i}$. We shall write $A^{i j}$ for $e^{i} A e^{j}=e^{i} \mathbf{A} ! e^{j}=\mathbf{A}^{i} \varphi^{i j}$. Define a $k_{t}$-linear endomorphism of $A_{t}$ by $f_{t}(x)=\sum e_{t}^{i} \cdot e^{i} x e^{j} \cdot e_{t}^{j}$. Note that $f_{t}\left(A^{i j}[[t]]\right)=e_{t}^{i} \cdot A_{t} \cdot e_{t}^{j}$. Since $f_{t}$ is the identity modulo $(t)$ it is an automorphism and we may use it to define an equivalent deformation $\mu_{t}$ by $f_{t} \mu_{t}(x, y)=f_{t} x \cdot f_{t} y$. Then $\mu_{t}$ is "upper triangular." That is, $\mu_{t}\left(A^{i j}, A^{j k}\right) \subseteq A^{i k}[[t]]$ and $\mu_{t}\left(A^{i j}, A^{k l}\right)=0$ if $j \neq k$. In particular, $\left.\mu_{t}\right|_{\mathbf{A}^{\prime}}$ gives a deformation $\mathbf{A}_{t}^{i}$ of $\mathbf{A}^{i}$.

Note that $\left\{e_{t}^{i}\right\}_{I}$ need not be a set of orthogonal idempotents under $\mu_{t}$; however, there will be some such set $\left\{\bar{e}_{t}^{i}\right\}_{I}$. Now each $e^{i}$ is the unit of $A^{i i}=\mathbf{A}^{i}$. Hence, $\bar{e}_{t}^{i}$ is the unit of $A_{t}^{i}$. Define another automorphism of $A[[t]]$ by $g_{t}(x)=\sum \bar{e}_{t}^{i} x \bar{e}_{t}^{j}$ and an equivalent deformation by $g_{t} \mu_{t}^{\prime}(x, y)=\mu_{t}\left(g_{t} x, g_{t} y\right)$. Then $\mu_{t}^{\prime}$ is again upper triangular and its restriction to the deformation of $\mathbf{A}^{i}$ has $e^{i}$ for its unit. Thus, we may take the $\left\{e^{i}\right\}_{I}$ themselves for the collection of orthogonal idempotents for $\mu_{t}^{\prime}$. Moreover, $1=\Sigma e^{i}$ is the unit for $\mu_{t}^{\prime}$.

For any diagram $\mathbf{A}$, a deformation $\mu_{t}=\Sigma \mu_{i} t^{i}$ of $\mathbf{A}$ ! will be called strict if each $\mu_{i}$ is a strict 2-cochain. The foregoing amounts to a proof of the following

THEOREM. If $\mathbf{A}$ is a diagram over a finite poset then every deformation of $\mathbf{A}$ ! is equivalent to a strict deformation.

21. Induced deformations. A deformation $\mathbf{A}_{t}$ of $\mathbf{A}$ naturally induces a deformation of $\mathbf{A}$ ! to $\mathbf{A}_{t} !=\prod_{i \in I} \amalg_{j \geqslant i} \mathbf{A}_{t}^{i} \varphi_{t}^{i j}$. Indeed, if $\Gamma_{n}$ is the $n$th cochain of $\mathbf{A}_{t}$ then $\tau \Gamma_{n}$ is that of $\mathbf{A}_{t}$ ! . Equivalent deformations of $\mathbf{A}$ clearly induce equivalent deformations of $\mathbf{A}$ ! - as do weakly equivalent deformations of $\mathbf{A}$. If we assume, as we may, that $e^{i}$ remains the unit of $\mathbf{A}_{t}^{i}$, then the units of $\mathbf{A}$ ! and $\mathbf{A}_{t}$ ! are the same. Observe then that there is a morphism of diagrams $\mathbf{K}_{t} \rightarrow \mathbf{A}_{t}$ where $\mathbf{K}_{t}$ is the trivial (and only) deformation of $\mathbf{K}$.

Now let $\mu$ represent the multiplication in $\mathbf{A}$ ! and $\mu_{t}$ that in some deformation $(\mathbf{A} !)_{t}$. As usual we may assume that 1 remains the unit for $\mu_{t}$; that is, $\mu_{t}=\mu+\Sigma_{m>0} \mu_{m} t^{m}$, where $\mu_{m}(1, x)=0=\mu_{m}(x, 1)$ for all $m$ and all $x \in \mathbf{A}$ !. If $\left.\mu_{m}\right|_{\mathbf{K} !}=0$ for all $m$, then $\mu_{t}\left(\varphi^{i j}, \varphi^{j k}\right)=\varphi^{i k}$ and, for $j \neq k, \mu_{t}\left(\varphi^{i j}, \varphi^{k l}\right)=0$. Hence there is an algebra morphism $\mathbf{K} !_{t} \rightarrow \mathbf{A} !_{t}$, where $\mathbf{K} !_{t}$ is the trivial deformation of $\mathbf{K}$ !, i.e., $\mathbf{K} !_{t}=\mathbf{K}_{t}$ !. The thrust of this section is to prove that when $I$ is finite every deformation of $(\# \mathbf{A})$ ! is equivalent to an induced one. Consequently, until the final theorem of this section there will be two standing assumptions: $I$ is finite and $H^{2}(\mathbf{K}, \mathbf{A})=0$ - the latter 
occurs, for example, if $I$ has a largest element (see §9). In this context we shall compare the deformation theories of $\mathbf{A}$ and $\mathbf{A}$ !.

Proposition. Any deformation (A!) is equivalent to a strict deformation satisfying

(i) $\left.\mu_{m}\right|_{\mathbf{K} !}=0$ for all $m$.

Proof. The results of $\S 20$ show that since $I$ is finite we may assume $\mu_{t}$ is strict. Suppose that for some $r \geqslant 0$ we have obtained an equivalent deformation $\mu_{t}^{(r)}=\mu+$ $\sum_{m>0} \mu_{m}^{(r)} t^{m}$ which is strict and satisfies $\left.\mu_{m}^{(r)}\right|_{\mathbf{K} !}=0$ for $m \leqslant r$. Then $\left.\mu_{r+1}^{(r)}\right|_{\mathbf{k}} \in$ $Z_{s}^{2}(\mathbf{K} !, \mathbf{A}$ !), while

$$
0=H^{2}(\mathbf{K}, \mathbf{A})=H^{2}(\mathbf{K} !, \mathbf{A} !)=H_{s}^{2}(\mathbf{K} !, \mathbf{A} !) .
$$

Hence $\left.\mu_{r+1}^{(r)}\right|_{\mathbf{K} !}+\delta g_{r+1}^{\prime}=0$ for some $g_{r+1}^{\prime} \in C_{s}^{1}\left(\mathbf{K}\right.$ !, $\mathbf{A}$ !). Define $g_{r+1} \in C_{s}^{1}(\mathbf{A}$ !, $\mathbf{A}$ !) by

$$
g_{r+1}\left(a^{i}, \varphi^{i j}\right)=a^{i} g_{r+1}^{\prime}\left(\varphi^{i j}\right) \text {. }
$$

Observe that

$$
g_{r+1}\left(\varphi^{i j}\right)=e^{i} g_{r+1}^{\prime}\left(\varphi^{i j}\right)=g_{r+1}^{\prime}\left(\varphi^{i j}\right)
$$

and, so, $\left.\left(\mu_{r+1}^{(r)}+\delta g_{r+1}\right)\right|_{\mathbf{K} !}=0$. Set $G_{t}^{(r+1)}=\mathrm{id}+g_{r+1} t^{r+1}$ and define $\mu_{t}^{(r+1)}$ by

$$
G_{t}^{(r+1)} \mu_{t}^{(r+1)}(x, y)=\mu_{t}^{(r)}\left(G_{t}^{(r+1)} x, G_{t}^{(r+1)} y\right) .
$$

Then it is easy to check that $\mu_{t}^{(r+1)}$ is strict. Also, $\mu_{m}^{(r+1)}=\mu_{m}^{(r)}$ for $m \leqslant r$ and $\mu_{r+1}^{(r+1)}=\mu_{r+1}^{(r)}+\delta g_{r+1}$; that is, $\left.\mu_{m}^{(r+1)}\right|_{\mathbf{k} !}=0$ for $m \leqslant r+1$. Hence this process may be iterated, beginning with $\mu_{t}^{(0)}=\mu_{t}$. The isomorphisms $G_{t}^{(r)}, r>0$, compose to give a well-defined power series $G_{t}$. Conjugating the given $\mu_{t}$ by $G_{t}$ then produces the required equivalent deformation. Indeed, it is $\mu+\Sigma \mu_{r}^{(r)} t^{r}$.

Lemma. Any deformation of A! is equivalent to a strict deformation satisfying (i) and (ii) $\mu_{m}\left(a^{i}, \varphi^{i j}\right)=0$ for all $m$ and all $a^{i} \varphi^{i j} \in \mathbf{A} !$.

Proof. The proposition shows that we may assume strictness and (i) at the outset. As before, suppose that for some $r \geqslant 0$ we have obtained an equivalent strict deformation $\mu_{t}^{(r)}$ satisfying (i) for all $m$ and (ii) for $m \leqslant r$. Define $h_{r+1} \in C_{s}^{1}(\mathbf{A}$ !, $\mathbf{A}$ !) by

$$
h_{r+1}\left(a^{i} \varphi^{i j}\right)=\mu_{r+1}^{(r)}\left(a^{i}, \varphi^{i j}\right)
$$

Then

$$
h_{r+1}\left(\varphi^{i j}\right)=\mu_{r+1}^{(r)}\left(e^{i}, \varphi^{i j}\right)=0 \quad \text { and } \quad h_{r+1}\left(a^{i}\right)=\mu_{r+1}^{(r)}\left(a^{i}, e^{i}\right)=0 .
$$

It follows that $\left(\mu_{r+1}^{(r)}+\delta h_{r+1}\right)\left(a^{i}, \varphi^{i j}\right)=0$. Using the $k_{t}$-linear automorphism $H_{t}^{r+1}$ $=\mathrm{id}+h_{r+1} t^{r+1}$, we define an equivalent strict deformation $\mu_{t}^{(r+1)}$ by

$$
H_{t}^{(r+1)} \mu_{t}^{(r+1)}(x, y)=\mu_{t}^{(r)}\left(H_{t}^{(r+1)} x, H_{t}^{(r+1)} y\right) .
$$

Then $\mu_{m}^{(r+1)}=\mu_{m}^{(r)}$ for $m \leqslant r$ and $\mu_{r}^{r+1}=\mu_{r+1}^{(r)}+\delta h_{r+1}$, giving (ii) for $m \leqslant r+1$. Moreover, since $H_{t}^{(r+1)}\left(\varphi^{i j}\right)=\varphi^{i j}$, we have $\left.\mu_{m}^{(r+1)}\right|_{\mathbf{K} !}=0$ for all $m$ (i.e., property (i)). Hence, as before, this process may be iterated, beginning with $\mu_{t}^{(0)}=\mu_{t}$. We obtain a 
sequence of isomorphisms $H_{t}^{(r)}, r>0$, which compose to give a well-defined power series $H_{t}$. Conjugating $\mu_{t}$ by $H_{t}$ then produces the required equivalent deformation.

Recall, from [G2], that the cochains of the deformation $\mu_{t}$ must satisfy the deformation equations $\delta \mu_{1}=0$ and $\delta \mu_{m}=\sum_{i=1}^{m-1} \mu_{i} \bar{\circ} \mu_{m-i}$.

Lemma. Any deformation of $\mathbf{A}$ ! is equivalent to a strict deformation satisfying

(iii) $\mu_{m}\left(x, y \varphi^{i j}\right)=\mu_{m}(x, y) \varphi^{i j}$ for all $m$ and all $x, y, \varphi^{i j} \in \mathbf{A} !$.

Proof. We may assume that the deformation is strict and that the cochains satisfy (i) and (ii). We show first, by induction, that $\mu_{m}\left(x, \varphi^{i j}\right)=0$ for all $m$ and all $x, \varphi^{i j} \in \mathbf{A}$ !.

If $\mu_{r}\left(x, \varphi^{i j}\right)=0$ for $r \leqslant m-1-\mathrm{a}$ vacuous condition when $m=1-$ the deformation equations show that $\delta \mu_{m}\left(a^{i}, \varphi^{i j}, \varphi^{k l}\right)=0$. But

$$
\begin{aligned}
\delta \mu_{m}\left(a^{i}, \varphi^{i j}, \varphi^{k l}\right)= & a^{i} \mu_{m}\left(\varphi^{i j}, \varphi^{k l}\right)-\mu_{m}\left(a^{i} \varphi^{i j}, \varphi^{k l}\right) \\
& +\mu_{m}\left(a^{i}, \varphi^{i j} \varphi^{k l}\right)-\mu_{m}\left(a^{i}, \varphi^{i j}\right) \varphi^{k l} .
\end{aligned}
$$

The first and fourth summands are zero due to the assumptions on $\mu_{m}$. The third is zero for the same reason if $j=k$ and because $\varphi^{i j} \varphi^{k l}=0$ otherwise. Thus, $\mu_{m}\left(a^{i} \varphi^{i j}, \varphi^{k l}\right)=0$ and, so, $\mu_{m}\left(x, \varphi^{i j}\right)=0$ for all $x, \varphi^{i j} \in \mathbf{A}$ !.

Next, the deformation equations imply $\delta \mu_{m}\left(x, y, \varphi^{i j}\right)=0$. But

$$
\begin{aligned}
\delta \mu_{m}\left(x, y, \varphi^{i j}\right) & =x \mu_{m}\left(y, \varphi^{i j}\right)-\mu_{m}\left(x y, \varphi^{i j}\right)+\mu_{m}\left(x, y \varphi^{i j}\right)-\mu_{m}(x, y) \varphi^{i j} \\
& =\mu_{m}\left(x, y \varphi^{i j}\right)-\mu_{m}(x, y) \varphi^{i j}
\end{aligned}
$$

and we have (iii).

LEMMA. A deformation of $\mathbf{A}$ ! is induced if and only if it is strict and satisfies (iii).

Proof. That an induced $\mu_{t}$ has these properties is clear if, as we have assumed, in any deformation of $\mathbf{A}$ the unit of $\mathbf{A}^{i}$ remains $e^{i}$. We prove the converse. Note that strictness and (iii) imply $\mu_{m}\left(x, \varphi^{i j}\right)=\mu_{m}\left(x, e^{i}\right) \varphi^{i j}=0$; so $\mu_{t}\left(x, \varphi^{i j}\right)=x \varphi^{i j}$, $\mu_{t}\left(\varphi^{i j}, \varphi^{j k}\right)=\varphi^{i k}$. Now strictness implies that $e^{i}$ remains the unit of $\mathbf{A}^{i}$ (hence 1 remains the unit of $\mathbf{A}$ !) and $\mu_{t}\left(\mathbf{A}^{i}, \mathbf{A}^{i}\right) \subset \mathbf{A}^{i}[[t]]$. We can therefore define the multiplication $\alpha_{t}^{i}$ in $\mathbf{A}^{i}[[t]]$ to be the restriction of $\mu_{t}$. Note, again using strictness, that $\mu_{t}\left(\varphi^{i j}, a^{j}\right) \subset \mathbf{A}^{i}[[t]] \varphi^{i j}$. Define $\varphi_{t}^{i j}: \mathbf{A}^{j}[[t]] \rightarrow \mathbf{A}^{i}[[t]]$ by $\varphi_{t}^{i j}\left(a^{j}\right) \varphi^{i j}=\mu_{t}\left(\varphi^{i j}, a^{j}\right)$. Then

$$
\begin{aligned}
\varphi_{t}^{i j}\left(\varphi_{t}^{j k}\left(a^{k}\right)\right) \varphi^{i k} & =\mu_{t}\left(\varphi^{i j}, \varphi_{t}^{j k}\left(a^{k}\right)\right) \varphi^{j k}=\mu_{t}\left(\varphi^{i j}, \varphi_{t}^{j k}\left(a^{k}\right) \varphi^{j k}\right) \\
& =\mu_{t}\left(\varphi^{i j}, \mu_{t}\left(\varphi^{j k}, a^{k}\right)\right)=\mu_{t}\left(\mu_{t}\left(\varphi^{i j}, \varphi^{j k}\right), a^{k}\right) \\
& =\mu_{t}\left(\varphi^{i k}, a^{k}\right)=\varphi_{t}^{i k}\left(a^{k}\right) \varphi^{i k} .
\end{aligned}
$$

A similar calculation shows that each $\varphi_{t}^{i j}$ is indeed an algebra morphism; so we have a deformation of $\mathbf{A}$ which, since $I$ is finite, induces the given $\mu_{t}$.

THEOREM. If I is finite then every deformation of $(\# \mathbf{A})$ ! is induced by a deformation of $\mathbf{A}$. The deformation theories of $(\# \mathbf{A})$ ! and $\mathbf{A}$ are equivalent if $\zeta_{\mathbf{A}}^{2}: H_{s}^{2}(\mathbf{A}, \mathbf{A}) \rightarrow$ $H^{2}(\mathbf{A}, \mathbf{A})$ is an isomorphism. This occurs, in particular, in the commutative case and when $\mathbf{A}$ is reduced to a single morphism. If $H^{2}(\mathbf{K}, \mathbf{A})=0$ then $(\# \mathbf{A})$ ! may be replaced by $\mathbf{A}$ !. This occurs, for example, if I has a largest element. 
Proof. The last two lemmas above prove the fourth statement; the fifth was proven in $\$ 9$. Together they show that every deformation of $(\mathbf{A})$ ! is induced by one of $\# \mathbf{A}$, since $\# I$ has a largest element. But the deformation theories of $\mathbf{A}$ and $\# \mathbf{A}$ are isomorphic $(\$ 20)$, giving the first claim.

For the second statement recall that sharpening $\mathbf{A}$ does not change the cohomology (\$7); so we may assume $I$ has a largest element. Let $\mathbf{A}_{t}$ and $\overline{\mathbf{A}}_{t}$ be two inequivalent deformations of $\mathbf{A}$; call their cochains $\left\{\Gamma_{r}\right\}$ and $\left\{\bar{\Gamma}_{r}\right\}$. We may assume (§8) that $\Gamma_{r}=\bar{\Gamma}_{r}, r<n$, and $\Gamma_{n}-\bar{\Gamma}_{n}$ is nonzero in $H_{s}^{2}(\mathbf{A}, \mathbf{A})$. Since $\zeta_{\mathbf{A}}^{2}$ (by assumption) and $\omega_{\mathbf{A}}^{2}$ (by the CCT) are isomorphisms we find $\tau \Gamma_{r}=\tau \bar{\Gamma}_{r}, r<n$, and $\tau \Gamma_{n}-\tau \bar{\Gamma}_{n}$ is a cocycle but not a coboundary. Hence $\mathbf{A}_{t}$ ! is not equivalent to $\overline{\mathbf{A}}_{t}$ !

The third statement was proven earlier $(\$ \S 3,7)$.

The appearance of (\#A)! in the above theorem is essential: $\mathbf{K}$ is rigid, while if the geometric realization of $I$ is, say a sphere, $H^{2}(\mathbf{K} !, \mathbf{K} !) \neq 0$ and $H^{3}(\mathbf{K} !, \mathbf{K} !)=0$ (§7); hence $\mathbf{K}$ ! has a nontrivial deformation.

Finally, note that this result can be obtained in many special cases without using the CCT. For example, in $\$ 20$ we proved it for diagrams over a finite poset with no dominance relations. We can also prove it directly when $\mathbf{A}$ is a morphism or a commutative square. The simplest intractable case occurs when the poset consists of five elements, two of which are maximal, and the dominance relations form two commutative squares.

22. Example: Some noncommutative varieties. The following is a special case of Theorems 8 and 12 of [G4]: Let $R$ be a ring and $D_{1}, D_{2}$ be two commuting derivations of $R$ into itself. If $R$ is an algebra over the rationals with multiplication $\pi$, then

$$
e^{t D_{1}-D_{2}}=\pi+t D_{1} \smile D_{2}+\left(t^{2} / 2 !\right) D_{1}^{2} \smile D_{2}^{2}+\cdots
$$

defines a deformation of $R$. ( $D_{1} \smile D_{2}$ is the usual cup product of cocycles.) If $R$ has characteristic $p>0$ and $D_{1}^{p}=D_{2}^{p}=0$, then the same is true of

$$
\pi+t D_{1} \smile D_{2}+\cdots+\left(t^{p-1} /(p-1) !\right) D_{1}^{p-1} \smile D_{2}^{p-1},
$$

which we continue to denote by $e^{t D_{1}-D_{2}}$. (It is not generally true that $e^{t D}=1+t D_{1}$ $+\cdots+\left(t^{p-1} /(p-1) !\right) D^{p-1}$ is an automorphism when $D^{p}=0$; one must have $D^{[(p-1) / 2]}=0$.) When $R=k[x, y]$ and $D_{1}=\partial_{x}=\partial / \partial x$ and $D_{2}=\partial y=\partial / \partial y$, then $e^{t \partial_{x}-\partial_{y}}$ is well defined without any conditions on $k$. For it is easy to check that if $f, g \in k[x, y]$ then $\partial_{x}^{n} f$ and $\partial_{y}^{n} g$ are both formally divisible by $n$ !, so the coefficient of $t^{n}$ in $e^{t \partial_{x}-\partial_{y}}(f, g)$ is not only integral but divisible by $n$ !.

Lemma. Let $S$ be any multiplicatively closed subset of $k[x, y]$ and set $R=S^{-1} k[x, y]$. Then $\partial_{x}^{n} r$ is divisible by $n$ ! for any $r \in R$.

Proof. If $r=f / s$, then by Leibniz' rule $\partial_{x}^{n} r=\Sigma\left(\begin{array}{c}n \\ m\end{array}\right) \partial_{x}^{n-m} f \partial_{x}^{m}\left(s^{-1}\right)$; so it is sufficient to show that $m$ ! divides $\partial_{x}^{m}\left(s^{-1}\right)$. This follows by induction on $m$ from Leibniz' rule and the equation $\partial_{x}^{m}\left(s s^{-1}\right)=0$ for $m \geqslant 1$.

It follows that $e^{t \partial_{x}-\partial_{y}}$ defines a deformation of every localization of $k[x, y]$, and the coefficient of $t^{n}$ in the series for $e^{t \partial_{x}-\partial_{y}}(a, b)$ is still divisible by $n$ ! in any such localization. The series thus converges in the topology obtained by taking as a 
fundamental set of neighborhoods of 0 the multiples of $n !$. In characteristic $p>0$ the series is a polynomial of degree $\leqslant p-1$ in $t$. Note that if $[x, y]_{t}$ denotes the commutator of $x$ and $y$ in the deformed multiplication, then $[x, y]_{t}=t$; this essentially defines the deformation.

We construct now a noncommutative deformation of projective 2-space $\mathbf{P}^{2}(k)$. Let $\mathbf{A}^{6}=k[x, y], \mathbf{A}^{5}=k\left[x_{1}, y_{1}\right], \mathbf{A}^{4}=k\left[x_{2}, y_{2}\right]$ be three copies of the polynomial ring in two variables over $k$. We view the variables as representing inhomogeneous coordinates in $\mathbf{P}^{2}$ whose homogeneous coordinates are $z_{0}, z_{1}, z_{2}$, where $(x, y)=$ $\left(z_{1} / z_{0}, z_{2} / z_{0}\right),\left(x_{1}, y_{1}\right)=\left(z_{0} / z_{1}, z_{2} / z_{1}\right)$, and $\left(x_{2}, y_{2}\right)=\left(z_{0} / z_{2}, z_{1} / z_{2}\right)$. Accordingly, we identify $k[x, y, 1 / x]$ with $k\left[x_{1}, y_{1}, 1 / x_{1}\right]$ by setting $x_{1}=1 / x, y_{1}=y / x$ and denoting this ring by $\mathbf{A}^{3}$. One then has inclusions $\varphi^{36}: \mathbf{A}^{6} \rightarrow \mathbf{A}^{3}$, and $\varphi^{35}$ : $\mathbf{A}^{5} \rightarrow \mathbf{A}^{3}$. Similarly, set

$$
\mathbf{A}^{2}=k\left[x_{1}, y_{1}, 1 / y\right]=k\left[x_{2}, y_{2}, 1 / y_{2}\right] \supseteq \mathbf{A}^{5}, \mathbf{A}^{4}
$$

and

$$
\mathbf{A}^{1}=k\left[x_{2}, y_{2}, 1 / x_{2}\right]=k[x, y, 1 / y] \supseteq \mathbf{A}^{4}, \mathbf{A}^{6} .
$$

Finally, set $\mathbf{A}^{0}=k[x, y, 1 / x, 1 / y]$, which contains all of the foregoing rings. Then $e^{t \partial_{x}-\partial_{y}}$ simultaneously defines deformations of all of these $\mathbf{A}^{i}$ to algebras $\mathbf{A}_{t}^{i}$ over $k[[t]]$. Moreover, $\varphi_{t}^{i j}$, the $k[[t]]-$ linear extension of $\varphi^{i j}$, remains a morphism for all $i \leqslant j$. We thus have deformed the original diagram $\mathbf{A}$ consisting of the $\mathbf{A}^{i}$ and $\varphi^{i j}$ to a diagram $\mathbf{A}_{t}$ representing, in some sense, a noncommutative $\mathbf{P}^{2}(k[[t]])$. (In characteristic $p$ we may use $k[t]$ and obtain noncommutative $\mathbf{P}^{2}(k)$ 's by substituting for $t$ the various elements of $k$.) When $k$ is a field, this "noncommutative" variety even has a "function skew-field (division ring)" $D_{t}$, namely the extension of the deformation to the full rational function field $k(x, y)$. (A deformation of a division ring and, in particular, of a field is always a division ring [G4].) If $k$ has characteristic $p>0$ then $k\left(x^{p}, y^{p}\right)$ is in the center of $D_{t}$; that center is then $Z_{t}=k\left(x^{p}, y^{p}\right)[[t]]$ and $D_{t}$ has dimension $p^{2}$ over $Z_{t}$.

Suppose now that we have fields $L \supseteq K$ where $L$ is a finite separable extension of $K$, and $D_{1}, D_{2}$ are commuting derivations of $K$ into itself. These have unique extensions $\bar{D}_{1}$ and $\bar{D}_{2}$ to $L$ which again commute, since $\left[\bar{D}_{1}, \bar{D}_{2}\right]$ and 0 are both derivations of $L$ which extend $\left[D_{1}, D_{2}\right]=0$. Similarly, if $K$ has characteristic $p>0$ and $D_{1}^{p}=D_{2}^{p}=0$, then also $\bar{D}_{1}^{p}=\bar{D}_{2}^{p}=0$ since $\bar{D}_{i}^{p}$ is a derivation extending $D_{i}^{p}$. A deformation $e^{t D_{1}-D_{2}}$ of $K$ can, therefore, be extended to $L$. (In [G4] there are more general deformation formulas which we shall not discuss here, but similar remarks apply.) The foregoing holds in particular for $K=k(x, y)$ with $k$ a field. The resulting deformation of $L$ is a division algebra which, when char $k=p>0$, has degree $p^{2}$ over its center $k L^{p}[[t]]$. (The more general formulae of [G4] give division algebras of higher degree.)

Suppose still that $k$ is a field and that we have an algebraic surface $\delta$ and a dominant separable morphism $f: \delta \rightarrow \mathbf{A}^{2}(k)=$ affine 2-space. If $K=k(x, y)$ is the function field of $\mathbf{A}^{2}(k)$ and $L$ that of $\mathcal{S}$, then the deformation $e^{t \partial_{x}-\partial y}$ of $K$ can be extended to $L$, since the hypothesis says that $L$ is a finite separable extension of $K$. However, $e^{t \partial_{x}-\partial_{y}}$ may not be defined on the ring $R$ of regular functions on $\delta$ since 
that ring need not be carried into itself by $\partial_{x}$ and $\partial_{y}$. Specifically, suppose that $z \in R$ and that $f(X) \in k[x, y][X]$ is an irreducible polynomial for $z$. Then a derivation $D$ of $k[x, y]$ can be extended to a derivation of $k[x, y, z]$ into $R$ if and only if $f^{d}(z)+f^{\prime}(z) u=0$ has a solution $u \in R$. ( $f^{d}$ is the polynomial obtained by applying $D$ to the coefficients of $f$.) Since $f^{\prime}(z) \neq 0$ the solution is unique if it exists, i.e., if $f^{D}(z) / f^{\prime}(z) \in R$. We can force it to exist by localizing $k[x, y]$ and $R$ at the multiplicatively closed set generated by $f^{\prime}(z)$. Since $R$ is finitely generated, to extend $\partial_{x}$ and $\partial_{y}$ we need do this only finitely often, so $e^{t \partial_{x}-\partial_{v}}$ can be extended from an open subset of $\mathbf{A}^{2}(k)$ to its inverse image in $\delta$. If we do this in different ways, getting different open subsets of $\mathbf{A}^{2}(k)$, then the deformations will agree on their intersection as will the extensions. Thus, there is a largest open subset of $\mathbf{A}^{2}(k)$ over which the deformation can be extended. Suppose now that we have a dominant morphism $f: S \rightarrow \mathbf{P}^{2}$. Then there is a largest open $U \subseteq \mathbf{P}^{2}$ such that the deformation previously given can be extended to $f^{-1} U$. Any dominant morphism from a surface $s$ to $\mathbf{P}^{2}$ therefore induces, in a natural way, a noncommutative structure on that part of $\bar{s}$ over some open subset of $\mathbf{P}^{2}$. In characteristic $p>0$ this deformation of $s$ is not merely formal. For $e^{t \partial_{1}-\partial_{1}}$ is a polynomial in $t$, both the deformed $\mathbf{P}^{2}$ and the deformed $s$ are defined over $k[t]$, and one can specialize $t$ to any value in $k$.

The restriction to surfaces in the foregoing is not essential. We can, for example, deform $\mathbf{A}^{n}(k)$, whose coordinate ring is $k\left[x_{1}, \ldots, x_{n}\right]$, by taking any subset $\left\{i_{1}, j_{1}, i_{2}, j_{2}, \ldots, i_{m}, j_{m}\right\}$ of $2 m$ distinct integers between 1 and $n$ and letting the new multiplication be $\exp \left(t \Sigma \partial_{i_{r}} \smile \partial_{j_{r}}\right)$, where $\partial_{i}=\partial / \partial x_{i}$ (cf. [G4]). This extends to a deformation of $\mathbf{P}^{n}(k)$. All of the foregoing then holds for any dominant separable morphism ${ }^{\top} \rightarrow \mathbf{P}^{n}$.

\section{REFERENCES}

[A] M. Andre, Rapport sur l'homologie des algèbres commutatives, 3ieme part., §16, Battelle Inst., Geneva, 1970.

[B] M. Barr, Harrison homologl, Hochschild homology and triples. J. Algebra 8 (1968), 314-323.

[Ba1] H. Bass, Algehraic K-theory, Benjamin, New York, 1968.

[Ba2] _. Introduction to some methods of algebraic $K$-theory, CBMS Regional Conf. Ser. in Math., No. 20, Amer. Math. Soc., Providence, R.I., 1974.

[F1] P. J. Fleury, Splitting of Hochschild's complex for commutative algehras, Proc. Amer. Math. Soc. 30 (1971), 405-411

[Fr] P. Freyd, Abelian categories, Harper \& Row, New York, 1964.

[FN] A. Froelicher and A. Nijenhuis, $A$ theorem on stability of complex structures. Proc. Nat. Acad. Sci. U.S.A. 43 (1957), 239-241.

[G1] M. Gerstenhaber, The cohomology structure of an associative ring. Ann. of Math. (2) 78 (1963), $267-288$.

[G2] _. On the deformation of rings and algehras, Ann. of Math. (2) 79 (1964), 59-103.

[G3] _ On the deformation of rings and algebras. II, Ann. of Math. (2) 84 (1966), 1-19.

[G4] On the deformation of rings and algebras. III, Ann. of Math. (2) 88 (1968), 1-34

[G5] . On the deformation of sheaves of rings, Global Analysis, papers in honor of K. Kodaira

(D. C. Spencer and S. Iyanaga, eds.), Tokyo Univ. Press, Tokyo, and Princeton Univ. Press, Princeton, N.J., 1969.

[G6] _. On the deformation of rings and algebras. IV, Ann. of Math. (2) 99 (1974), 257-276.

[Ha] D. K. Harrison, Commutative algebras and cohomologv. Trans. Amer. Math. Soc. 104 (1962), 191-204.

[Ho] G. Hochschild, On the cohomologv groups of an associative algebra. Ann. of Math. (2) 46 (1945), $58-67$. 
[K1] K. Kodaira, $A$ theorem of completeness of characteristic systems for analytic families of compact submanifolds of complex manifolds, Ann. of Math. (2) 75 (1962), 146-162.

[K2] , On stability of compact submanifolds of complex manifolds, Amer. J. Math. 85 (1963), 79-94.

[KS1] K. Kodaira and D. C. Spencer, On deformations of complex analytic structures. I. II. Ann. of Math. (2) 67 (1958), 328-466.

[KS2] _ On deformations of complex analytic structures. III, Ann. of Math. (2) 71 (1960), 43-76.

[LS] S. Lichtenbaum and M. Schlessinger. The cotangent complex of a morphism, Trans. Amer. Math. Soc. 128 (1967), 41-70.

[M] S. Mac Lane, Homologv, Academic Press, New York, 1963.

[N] A. Nijenhuis, A Lie product for the cohomology of subalgebras with coefficients in the quotient, Bull. Amer. Math. Soc. 73 (1967), 962-967.

[NR] A. Nijenhuis and R. W. Richardson, Jr.. Deformations of homomorphisms of Lie groups and Lie algebras, Bull. Amer. Math. Soc. 73 (1967), 175-179.

[Rch] R. W. Richardson, Jr., A rigidity theorem for subalgebras of Lie and associative algebras, Illinois J. Math. 11 (1967), 92-110.

[Rm] D. S. Rim, Formal deformation theory, Groupes de Monodromie en Géométrie Algébrique (SGA 7 I), Lecture Notes in Math., vol. 288, Springer-Verlag, Berlin and New York, 1972.

[Sch] S. D. Schack, On the deformation of an algebra homomorphism, Thesis, Univ. of Pennsylvania. 1980.

[Scl] M. Schlessinger, Functors of Artin rings, Trans. Amer. Math. Soc. 130 (1968), 208-222.

[Su] D. Sundararaman, Moduli, deformations and classifications of compact complex manifolds, Pitman, Boston, 1980.

Department of Mathematics, University of Pennsylvania, Philadelphia, Pennsylvania 19104 (Current address of M. Gerstenhaber)

Current address (S. D. Schack): Department of Mathematics, State University of New York, Buffalo, New York 14214 